\title{
Current Advances in Colorectal Cancer Treatment
}

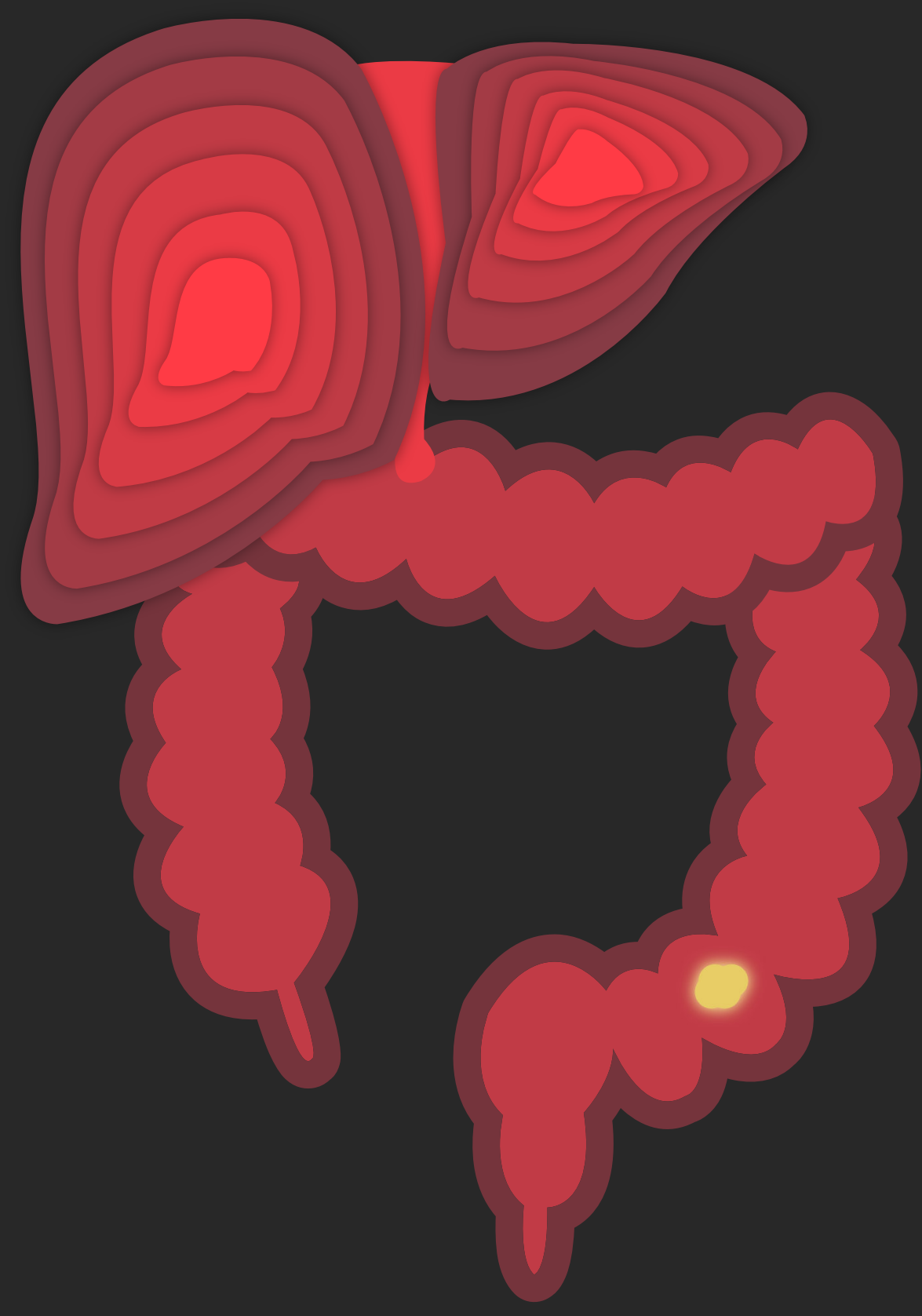

Esther Kok - de Goede 


\section{CURRENT ADVANCES IN COLORECTAL CANCER TREATMENT}

Esther Nicole Daniëlle Kok - de Goede 
This dissertation was approved by:

\section{Supervisor}

Prof. dr. T.J.M. Ruers

Co-supervisor

Dr. K.F.D. Kuhlmann

Cover design: Tevin Stuurland

Lay-out: $\quad$ E.N.D. Kok - de Goede

Printed by: $\quad$ ProeftschriftMaken || www.proefschriftmaken.nl

ISBN: $\quad$ 978-90-365-4989-9

DOI: $\quad 10.3990 / 1.9789036549899$

URL: $\quad$ https://doi.org/10.3990/1.9789036549899

(c) 2020, E.N.D. Kok - de Goede, The Netherlands.

All rights reserved. No parts of this thesis may be reproduced, stored in a retrieval system or transmitted in any form or by any means without permission of the author. Alle rechten voorbehouden. Niets uit deze uitgave mag worden vermenigvuldigd, in enige vorm of op enige wijze, zonder voorafgaande schriftelijke toestemming van de auteur. 


\title{
CURRENT ADVANCES IN COLORECTAL CANCER TREATMENT
}

\section{DISSERTATION}

To obtain

the degree of doctor at the University of Twente, on the authority of the rector magnificus, prof. dr. T.T.M. Palstra, on account of the decision of the Doctorate Board, to be publicly defended on Friday June $19^{\text {th }}, 2020$ at 12.45 hours

$$
\text { by }
$$

\section{Esther Nicole Daniëlle Kok - de Goede}

\author{
born on October $19^{\text {th }}, 1989$ \\ in Amersfoort, the Netherlands
}




\section{Graduation Committee}

Chairman/secretary

Prof. dr. J.L. Herek

Supervisor

Prof. dr. T.J.M. Ruers

Co-supervisor

Dr. K.F.D. Kuhlmann

Committee Members

Prof. dr. I.A.M.J. Broeders

Prof. dr. Ir. C.H. Slump

Prof. dr. G.L. Beets

Prof. dr. J.M. Klaase

Dr. C.F.J.M. Blanken - Peeters
University of Twente

University of Twente / Antoni van Leeuwenhoek Netherlands Cancer Institute

Antoni van Leeuwenhoek - Netherlands Cancer Institute

University of Twente

University of Twente

University of Maastricht

University of Groningen

Rijnstate Arnhem 


\section{Table of Contents}

$\begin{array}{lll}\text { Chapter } 1 & \text { Introduction and Outline }\end{array}$

Chapter $2 \quad$ Multicentre study of short-course radiotherapy, 22

systemic therapy and resection/ablation for stage IV

rectal cancer

Chapter 3 High versus low dose Stereotactic Body Radiation

Therapy for hepatic metastases

Chapter 4 Accelerated growth of synchronous colorectal liver metastases after primary tumor resection: effects of neoadjuvant therapy

Chapter 5 Hyperspectral imaging for tissue classification, a way toward smart laparoscopic colorectal surgery

Chapter 6 Association of image-guided navigation with complete resection in patients with locally advanced primary and recurrent rectal cancer

Chapter 7 Accurate surgical navigation with real-time tumor tracking in cancer surgery

Chapter $8 \quad$ General Discussion

PhD Portfolio

Dankwoord 



\section{Chapter 1}

General introduction 


\section{Statistics}

Colorectal cancer (CRC) is the third most commonly diagnosed cancer worldwide. In the Netherlands, approximately 15.000 patients are diagnosed with CRC annually, of which 4.400 patients with rectal cancer [1]. The lifetime incidence of CRC is around 5\% and increases with age [2]. Although the incidence doubled over the last three decades, mortality rates remained relatively stable throughout the years. The five-year relative survival rate is strongly dependent on the stage at diagnosis and varies from $92-95 \%$ for stage I to $11-14 \%$ for stage IV disease [1]. Despite improved treatment modalities, CRC remains the second leading cause of cancer-related death, accounting for 6.000 deaths per year in the Netherlands [3].

\section{Diagnosis and staging}

Patients with CRC may present with clinical symptoms or can be identified by routine screening. Once CRC is suspected, the diagnostic workup consists of a physical examination, bloodwork, colonoscopy, and imaging of the abdomen and chest. Colonoscopy is the most accurate test to localize the tumor and allows for taking biopsies for histopathological confirmation. For diagnostic imaging, a computed tomography (CT) scan is acquired for regional tumor extension, regional lymphatic and distant metastases. Nowadays, magnetic resonance imaging (MRI) is preferred over CT scanning for diagnosing liver metastases since it has a higher sensitivity [4]. In rectal cancer patients, an additional pelvic MRI scan is performed to determine the accurate tumor location and extension.

According to a recently published classification, staging between rectal and sigmoid tumors is defined by the sigmoid take-off, as determined on imaging. Tumors with the lower border below the sigmoid take-off are classified as rectal tumors and tumors with the lower border above the sigmoid take-off as sigmoid tumors [5]. The staging system is furthermore based on the size and extent of the primary tumor ( $T$ ), regional lymph node involvement (N) and the occurrence of distant metastases (M) [6]. The TNM-staging provides an overall clinical stage and is indicative for overall survival, as shown in Table 1. The higher the stage, the further the disease has progressed, with stage IV disease - indicating distant metastases, independent of the primary tumor and nodal stage - as the highest stage. Metastases can present at the same time as the primary tumor (synchronous metastases) or delayed, mostly after the primary tumor has been treated (metachronous metastases) [7]. The most common sites for distant colorectal metastases are the liver, lungs, and peritoneum. The TNM-staging is, besides 
Table 1. TNM classification of CRC (7th edition AJCC)

\begin{tabular}{|c|c|c|c|c|c|}
\hline \multirow[t]{2}{*}{ Stage } & \multirow[t]{2}{*}{$\mathbf{T}^{1}$} & \multirow[t]{2}{*}{$\mathbf{N}^{2}$} & \multirow[t]{2}{*}{$M^{3}$} & \multicolumn{2}{|c|}{ Relative 5-year survival } \\
\hline & & & & Colon & Rectum \\
\hline 1 & $\mathrm{~T} 1-2$ & No & Mo & $92 \%$ & $94 \%$ \\
\hline IIA & T3 & No & Mo & $85 \%$ & $77 \%$ \\
\hline IIB & T4 & No & Mo & & \\
\hline IIIA & $\mathrm{T} 1$ & $\mathrm{~N} 1$ & Mo & $68 \%$ & $65 \%$ \\
\hline \multirow[t]{2}{*}{ IIIB } & $\mathrm{T} 1-2$ & N2 & MO & & \\
\hline & T3 & N1 & Mo & & \\
\hline \multirow[t]{2}{*}{ IIIC } & $\mathrm{T} 4$ & $\mathrm{~N} 1$ & MO & & \\
\hline & Any $T$ & $\mathrm{~N} 2$ & Mo & & \\
\hline IV & Any $T$ & Any N & M1 & $12 \%$ & $13 \%$ \\
\hline
\end{tabular}

'T1: tumor invades the submucosa. T2: tumor invades the muscularis propria. T3: tumor invades the pericolorectal tissue. T4: tumor invades the visceral peritoneum or other adjacent organs.

${ }^{2} \mathrm{~N} 0$ : no regional lymph node metastases. N1: metastases in $1-3$ regional lymph nodes. N2: metastases in $\geq 4$ regional lymph nodes.

${ }^{3} \mathrm{MO}$ : No evidence of distant metastases. M1: disease spread to distant organs

for overall survival, important for the prediction of the prognosis, treatment planning, and monitoring treatment response.

\section{Treatment}

All patients should be discussed by a specialized multidisciplinary team including radiologists, gastroenterologists, radiation- and medical oncologists and surgeons. Studies showed that the involvement of a multidisciplinary team results in optimal multidisciplinary treatment and improves survival in CRC patients [9, 10]. The most common treatment modalities in CRC are radiotherapy, chemotherapy, and surgery, of which surgery is the cornerstone of treatment. Chemotherapy can be given before (neoadjuvant) or after (adjuvant) surgery. The aim of neoadjuvant therapy is mostly risk reduction of local recurrence and improvement of resectability. The aim of adjuvant therapy is to eradicate tumor micrometastases after surgical resection. Treatment planning is highly dependent on the location of the primary tumor and the stage of the disease.

\section{Localized colon cancer}

In localized colon cancer, treatment is based on upfront surgical resection followed by adjuvant chemotherapy, depending on the clinical stage [11]. In case of a small stage I tumor, local excision without adjuvant treatment is considered safe [12]. For other stage I cancers or low-risk stage II disease, surgical resection without adjuvant therapy 
is recommended. Depending on the location of the tumor, a hemicolectomy (left or right) or a partial colectomy can be performed. Patients with high-risk stage II (cT4) and stage III disease should be offered 3 months of chemotherapy 4 to 8 weeks after surgical resection. In stage II this reduces the risk of death by $3 \%-5 \%$ and in stage III by $15 \%-20 \%$ [13]. Currently, there is no consensus regarding neoadjuvant chemotherapy in patients with localized colon cancer.

\section{Localized rectal cancer}

In localized rectal cancer, radiotherapy and surgery are the main treatment modalities [11]. Treatment is dependent on the stage and divided into 3 categories: early, intermediate and advanced stage rectal cancer. Early-stage is defined by cT1-3N0, extramural invasion $\leq 5 \mathrm{~mm}$, distance to MRF $>1 \mathrm{~mm}$. Intermediate-stage includes patients with $\mathrm{cT} 3 \mathrm{cdN} 0$ or $\mathrm{cT} 1-3$ (MRF-)N1. Advanced-stage includes patients with cT2-3, distance to MRF $\leq 1 \mathrm{~mm}$, or cT4 with $\geq 4$ lymph mesorectal lymph nodes or positive nodes outside the mesorectum. In early-stage rectal carcinoma, surgical resection without preoperative treatment is recommended. Total mesorectal excision is the standard of care, and depending on the location of the tumor, a low anterior resection (LAR) or abdominoperineal resection (APR) can be performed. In patients with intermediate-stage rectal cancer, short-course radiotherapy (5x5 Gy) followed by resection should be discussed. The recommended treatment in patients with advanced-stage rectal cancer is neoadjuvant long-term chemoradiation (25 x 2 Gy with capecitabine) followed by surgical resection. As an alternative, neoadjuvant shortcourse radiotherapy ( $5 \times 5 \mathrm{~Gy}$ ) can be considered. The randomized study of Bujko et al. compared short-course radiotherapy combined with 3 cycles of chemotherapy with long-course chemoradiation in patients with locally advanced rectal cancer and no significant difference was found in local efficacy between these treatments [14].

\section{Local treatment metastatic disease}

Approximately $50 \%$ of CRC patients will encounter metastastic disease and in around $80 \%$ they will occur in the liver [15]. Other possible locations are in the lung, bone and peritoneum. Treatment of metastases depends on the extent of disease, location and the timing of the appearance. Approximately $25 \%$ of the patients have synchronous distant metastases at diagnosis and another $25 \%$ will develop metachronous distant metastases during follow up [7]. In the current thesis, only treatment options for liver metastases will be discussed.

Available curative local treatment modalities for liver metastases are surgical resection, 
thermal ablation, and radiotherapy. Of these treatments, surgical resection remains the gold standard. Over the past decades, the number of liver resections increased due to a higher incidence of CRC and the expanding criteria for resection [16]. Whether surgical resection is feasible depends on the expected remnant liver, location of the tumor(s) with respect to the vascular and biliary structures and the performance state of the patient [17]. In patients with a normal liver function, the remaining liver volume after resection must be at least $30 \%$ of the preresection liver volume [18]. Over time, surgical mortality rates after hepatic resection decreased to $<5 \%$ while long-term survival rates increased from approximately $25 \%$ to $45 \%$ [19-26].

Besides surgical resection, thermal ablation with radiofrequency ablation (RFA) and microwave ablation (MWA) have proven to be successful treatment modalities for hepatic malignancies [27-29]. Several studies performed RFA as primary treatment with acceptable local control rates, especially in tumors smaller than $3 \mathrm{~cm}$ and with an ablation margins larger than $5 \mathrm{~mm}[30,31]$. There are no randomized trials comparing thermal ablation with surgical resection, although currently a randomized trial is ongoing (ClinicalTrials.gov Identifier: NCT03088150). Retrospective comparisons suggest that lower local recurrence rates and better survival are observed after resection in a general population of patients with liver metastases [32-34]. In certain patients, however, e.g. with a small central located tumor, there may be a clear indications for ablation instead of resection.

A relatively new curative local treatment for liver metastases is stereotactic body radiation therapy (SBRT). In the past, the role of radiation for liver metastases was limited due to toxicity and the risk of radiation-induced liver disease [35]. But in the last decade, SBRT developed largely and some phase I and II studies showed that this is a safe and accurate technique [36-38]. Compared to surgery and thermal ablation, this is a noninvasive treatment and no hospital admission is required. As with ablation, mainly patients unsuitable for surgical resection receive SBRT currently. Despite the increasing amount of studies, the ultimate dose and fractionation schedule for liver metastases remains unclear.

\section{Colorectal cancer with synchronous metastatic disease}

In patients with synchronous stage IV CRC, a multimodality therapy is necessary. The optimal approach remains unclear and depends on the extent of disease, symptoms, patient condition and experience per center. Treatment of synchronous liver metastases is typically more complex compared to metachronous liver metastases since it 
involves two tumor locations: the primary tumor and the metastases. Treatment is less standardized and for each individual patient, a personalized treatment plan must be made.

In patients with initially resectable synchronous metastatic $C R C$, surgical resection of the primary tumor and metastatic disease is the treatment of choice. In patients with isolated metastases in the liver, surgical resection of all metastatic sites can provide long-term survival [22]. The procedure can be simultaneous or staged, with the option for a colorectal first (the classic approach) or liver-first approach. Systematic reviews and meta-analyses found no significant difference in surgical outcome or survival between these approaches [39-41]. The best postoperative strategy remains controversial [42]. Unfortunately, the majority of the patients with synchronous stage IV CRC do not have resectable / ablatable metastases when diagnosed.

Patients with metastatic disease that may become resectable after chemotherapy are often referred to as potentially resectable patients. Patients with potentially resectable metastatic disease receive upfront systemic therapy in order to downsize the metastases and enable/improve resectability. Previous studies showed that tumors might become resectable in $15-60 \%$ of the patients but the optimal systemic therapy regimen remains unknown [43-45]. Currently, a Dutch multicenter randomized phase 3 trial (CAIRO5) compares multiple systemic treatment regimens in CRC patients with initially unresectable liver-only metastases (NCT02162563) [46]. In patients with a good response with secondary resectable metastases, surgery should follow.

In rectal cancer patients with potentially resectable metastases, the dilemma exists what tumor site should be treated first. In the setting of synchronous metastases, the rectal tumor is often locally advanced, where traditionally the preferred treatment is long-course chemoradiation including capecitabine or 5-fluorouracil [47]. However, the chemotherapy only works as a radiation sensitizer and has limited effect on the metastases, leaving them untreated for a significant minimally. Alternatively, starting with systemic therapy would leave the primary tumor suboptimal treated since the effect of systemic therapy on rectal cancer is limited. A possible treatment schedule has been published in a phase II trial of van Dijk et al. and includes preoperative short-course radiotherapy ( $5 \times 5 \mathrm{~Gy}$ ), followed by six cycles of capecitabine-oxaliplatin-bevacizumab (CAPOX-B), with the intent of subsequent surgical resection or ablation of all tumor sites, rectal and liver $[48,49]$. An alternative treatment schedule includes chemotherapy followed by a liver-first approach, with subsequent (chemo-)radiotherapy and primary 
tumor resection $[50,51]$. Both treatments showed to be safe and effective and have been implemented in multiple centers in the Netherlands.

Unfortunately, in many patients with stage IV disease, the metastases remain unresectable. In these patients, the therapeutic goal is to preserve the quality of life and prolong survival [52]. Treatment can consist of systemic therapy, radiotherapy or palliative surgery. In the last decades, palliative systemic therapy has increased overall survival significantly [53]. In patient with symptomatic rectal tumors, palliative pelvic radiotherapy can be given to relieve symptoms like pain, hemorrhage, and obstruction but the ultimate radiotherapy regimen remains unclear [54]. Furthermore, the role of primary tumor resection in patients with unresectable colorectal liver metastases remains under debate. Some studies compared primary tumor resection with chemotherapy alone and found no significant difference in overall survival [5557]. Others argue that resection is only required when tumor-related symptoms like obstruction, perforation or bleeding occur [58]. In contrast, many studies demonstrated the benefits of removal of the primary tumor in patients with unresectable liver metastases [59-64]. It can prevent tumor-related complications and is associated with improved overall survival $[65,66]$.

\section{Technical innovations to improve surgical outcomes}

In the past decades, the treatment of CRC patients has improved largely due to advances in imaging, neoadjuvant therapy, and surgery. These advancements improved survival significantly in CRC patients [67]. Intraoperatively, multiple new techniques have been developed to improve the accuracy and safety of surgical procedures. Laparoscopic, endoscopic and robotic surgery are examples of surgical innovations that evolved rapidly and are currently well established in fields like urology, gynecology and surgical oncology. In colorectal surgery, laparoscopic surgery showed improved short-term surgical outcomes with similar recurrence and long-term survival rates compared to open surgery in multiple randomized controlled trials [68-70]. However, some disadvantages of minimally invasive surgery must be acknowledged. First, the lack of tactile feedback makes tissue recognition during the procedure more challenging. Second, visualization and orientation can be difficult due to the moving camera and the two-dimensional screen.

In spite of improved imaging and surgical techniques, high rates of positive resection margins are still observed in cancer surgery [71]. Therefore, additional imaging techniques are being explored to improve intraoperative visualization. The ultimate 
goal is to increase complete removal rates and decrease damage to surrounding tissue. This should lead to better survival and increase quality of life. Image-guided navigation, fluorescence imaging, and hyperspectral imaging are examples of imaging techniques that might improve visualization during surgery and therefore surgical outcomes. For an extensive overview of these techniques, we refer to references [72-78]. In this thesis, the possibilities of image-guided navigation and hyperspectral imaging in colorectal surgery are evaluated.

\section{Hyperspectral imaging}

Hyperspectral imaging ( $\mathrm{HSI}$ ) has been used for various applications, e.g. planetary exploration, bloodstain visualization in crime scenes, art conservation and environmental monitoring [79-82]. It was first introduced in the medical field in the 1990s. In the past decades, its use in tissue perfusion, characterization of skin bruises, and as a diagnostic tool in cancer has been evaluated [83-88]. Previous studies showed that tissue classification with HSI is feasible in head and neck, breast and colon cancer [89-91]. In resected breast specimens, the accuracy to distinguish invasive carcinoma from healthy tissue is 93\% [90]. In CRC, HSI has been used on hematoxylin-eosin pathology slides and for tissue classification during endoscopy $[92,93]$. In the current thesis, the feasibility of HSI to differentiate healthy colorectal tissue from tumor tissue in a surgical setting will be evaluated.

Hyperspectral imaging combines optical spectroscopy with digital imaging. The technique uses a light source to illuminate an object. The light interacts with the object (reflection, scattering and/or absorption of the photons) after which it reflects back to the surface, where it is detected by the hyperspectral camera. For each pixel in the image, a hypercube is obtained. This hypercube has 3 dimensions, including spatial information (a reference to the specific location in the image) in two dimensions and wavelengths in the third dimension. In other words, a diffuse reflectance spectrum is obtained for each pixel. The shape and magnitude of the spectra are representative for tissue properties. With this information, healthy tissue can be differentiated from tumor tissue. Since the measurements can be performed fast, without direct tissue contact or exogenous contrast, this technique could be of great value for resection margin assessment in rectal surgery.

\section{Image-guided navigation}

Image-guided navigation is a surgical technique that matches preoperative imaging with the corresponding intraoperative setting, allowing surgeons to visualize their 
surgical instruments with respect to the surgical anatomy. This technique has evolved rapidly over the past two decades. It was first developed in neurosurgery with the aim to perform safer and less invasive surgical procedures. Because of the real-time visualization of surgical instrumentation with respect to intraoperative anatomy, it facilitates precise localization of tumor borders and critical healthy surrounding structures. Neurosurgery was the first to integrate it successfully into clinical care. A randomized controlled trial showed that the navigation technique improved complete tumor resections and improved progression-free survival [94]. The clinical application of this technique expanded subsequently to fields like orthopedic, trauma, and head and neck surgery. In these fields, navigation also showed to be of additional value since it improved accurate placement of surgical implants $[95,96]$. To investigate whether surgical navigation would be possible in abdominal surgery, our research group developed a novel electromagnetic surgical navigation system for pelvic malignancies. In a feasibility study, this in-house developed navigation system showed to be safe and feasible with high accuracies $(4.0 \mathrm{~mm})$ [97].

Image-guided navigation consists of a sequence of steps. In short, preoperative images are acquired and used to make a patient-specific 3D model. In the operation room, the preoperative imaging is linked to the intraoperative patient anatomy. During the procedure, a tracking system is used to display the position of the surgical instruments in the 3D model. With this technique, surgeons might perform more adequate tumor resection while sparing critical structures. This could improve the safety of surgical procedures and might even increase survival.

\section{Aim and outline of this thesis}

The general aim of this thesis is to examine current advances in the treatment of colorectal cancer. The thesis investigates the results of different novel treatment modalities for metastastic CRC patients in chapters 2 to 4 . Additionally, the development of new surgical techniques for CRC surgery are evaluated in chapters 5 to 7.

The first part of this thesis is focused on new application of existing treatment in patients with stage IV CRC. Chapter 2 evaluates the feasibility and effectiveness of short-course pelvic radiotherapy ( $5 \times 5 \mathrm{~Gy}$ ) followed by systemic therapy and local treatment of all tumor sites in potentially curable stage IV rectal cancer patients in daily practice. A multicenter retrospective study is performed, evaluating the completion rate of the full treatment schedule, symptom control of the primary tumor and survival. 
In Chapter 3, high dose Stereotactic Body Radiation Therapy (SBRT) is compared to low dose SBRT for hepatic metastases. The aim of this study is to evaluate the efficacy and safety of high versus low dose SBRT in patients with liver metastases in our institute and to determine prognostic variables on local control and overall survival. Chapter 4 describes a prospective, randomized controlled pilot study conducted in the Netherlands Cancer Institute and examines if neoadjuvant chemotherapy and/or targeted therapy in patients with unresectable liver metastases can inhibit the growth of the metastases induced by the primary tumor resection.

The second part of this thesis is focused on new surgical techniques for localized CRC surgery. Chapter 5 includes an ex vivo study investigating the use of hyperspectral imaging for tissue classification in CRC. Tissue samples from CRC surgery were imaged with two hyperspectral cameras. The ultimate goal is to develop a real-time technique for tissue identification in colorectal surgery. In Chapter 6 and 7, a different intraoperative imaging technique is evaluated. Chapter 6 evaluates whether imageguided navigation during locally advanced primary or recurrent rectal cancer surgery can improve resection margin rates compared to rectal surgery without navigation. The study compares the clinical outcomes of patients from a prospective, single-arm study with patients from a historical cohort. Chapter 7 explores the feasibility of realtime tumor tracking with image-guided navigation in patients with non-fixed mobile rectal tumors. An electromagnetic tracking system is used to real-time track the rectal tumor during the course of surgery.

The last part of this thesis includes the general discussion with a summary and the future perspective (Chapter 8). 


\section{References}

1. IKNL. Bowel Cancer. 2018 26-11-2019]; Available from: http://www.cijfersoverkanker.nl.

2. Siegel, R.L., K.D. Miller and A. Jemal, Cancer statistics, 2019. CA Cancer J Clin, 2019. 69(1): p. 7-34

3. Bray, F., J. Ferlay, I. Soerjomataram, et al., Global cancer statistics 2018: GLOBOCAN estimates of incidence and mortality worldwide for 36 cancers in 185 countries. CA Cancer J Clin, 2018.

4. Niekel, M.C., S. Bipat and J. Stoker, Diagnostic imaging of colorectal liver metastases with CT, MR imaging, FDG PET, and/or FDG PET/CT: a meta-analysis of prospective studies including patients who have not previously undergone treatment. Radiology, 2010. 257(3): p. 674-684.

5. D'Souza, N., M.P.M. de Neree Tot Babberich, A. d'Hoore, et al., Definition of the Rectum: An International, Expert-based Delphi Consensus. Ann Surg, 2019. 270(6): p. 955-959.

6. Edge, S.B. and C.C. Compton, The American Joint Committee on Cancer: the 7th edition of the AJCC cancer staging manual and the future of TNM. Ann Surg Oncol, 2010. 17(6): p. 1471-1474.

7. van der Pool, A.E., R.A. Damhuis, J.N. Ijzermans, et al., Trends in incidence, treatment and survival of patients with stage IV colorectal cancer: a population-based series. Colorectal Dis, 2012. 14(1): p. 56-61.

8. Brouwer, N.P.M., A. Bos, V. Lemmens, et al., An overview of 25 years of incidence, treatment and outcome of colorectal cancer patients. Int J Cancer, 2018. 143(11): p. 2758-2766.

9. MacDermid, E., G. Hooton, M. MacDonald, et al., Improving patient survival with the colorectal cancer multi-disciplinary team. Colorectal Dis, 2009. 11(3): p. 291-295.

10. Prades, J., E. Remue, E. van Hoof, et al., Is it worth reorganising cancer services on the basis of multidisciplinary teams (MDTs)? A systematic review of the objectives and organisation of MDTs and their impact on patient outcomes. Health Policy, 2015. 119(4): p. 464-474.

11. Richtlijnen database - Colorectaal carcinoom (CRC). 29-10-2019 20-01-2020]; Available from: https:// richtlijnendatabase.nl/richtlijn/colorectaal_carcinoom_crc/startpagina_-_crc.html\#algemeen.

12. Bhangu, A., G. Brown, R.J. Nicholls, et al., Survival outcome of local excision versus radical resection of colon or rectal carcinoma: a Surveillance, Epidemiology, and End Results (SEER) populationbased study. Ann Surg, 2013. 258(4): p. 563-9; discussion 569-571.

13. Schmoll, H., E. Van Cutsem, A. Stein, et al., ESMO Consensus Guidelines for management of patients with colon and rectal cancer. a personalized approach to clinical decision making. 2012. 23(10): p. 2479-2516.

14. Bujko, K., L. Wyrwicz, A. Rutkowski, et al., Long-course oxaliplatin-based preoperative chemoradiation versus $5 \times 5$ Gy and consolidation chemotherapy for cT4 or fixed cT3 rectal cancer: results of a randomized phase III study. Ann Oncol, 2016. 27(5): p. 834-842.

15. Manfredi, S., C. Lepage, C. Hatem, et al., Epidemiology and management of liver metastases from colorectal cancer. Ann Surg, 2006. 244(2): p. 254-259.

16. de Ridder, J.A.M., V.E.P.P. Lemmens, L.I.H. Overbeek, et al., Liver Resection for Metastatic Disease; A Population-Based Analysis of Trends. Digestive Surgery, 2016. 33(2): p. 104-113.

17. Alberts, S.R. and G.J. Poston, Treatment advances in liver-limited metastatic colorectal cancer. Clin Colorectal Cancer, 2011. 10(4): p. 258-265.

18. Vauthey, J.N., M.A. Choti and W.S. Helton, AHPBA/SSO/SSAT Consensus Conference on hepatic colorectal metastases: rationale and overview of the conference. January 25, 2006. Ann Surg Oncol, 2006. 13(10): p. 1259-1260.

19. Fong, Y., J. Fortner, R.L. Sun, et al., Clinical score for predicting recurrence after hepatic resection for metastatic colorectal cancer: analysis of 1001 consecutive cases. Ann Surg, 1999. 230(3): p. 309-18; discussion 318-321.

20. Nordlinger, B., M. Guiguet, J.C. Vaillant, et al., Surgical resection of colorectal carcinoma metastases to the liver: a prognostic scoring system to improve case selection, based on 1568 patients. Cancer, 1996. 77(7): p. 1254-1262.

21. De Jong, M.C., C. Pulitano, D. Ribero, et al., Rates and patterns of recurrence following curative intent surgery for colorectal liver metastasis: an international multi-institutional analysis of 1669 patients. 
2009. 250(3): p. 440-448.

22. Kanas, G.P., A. Taylor, J.N. Primrose, et al., Survival after liver resection in metastatic colorectal cancer: review and meta-analysis of prognostic factors. Clin Epidemiol, 2012. 4: p. 283-301.

23. Creasy, J.M., E. Sadot, B.G. Koerkamp, et al., Actual 10-year survival after hepatic resection of colorectal liver metastases: what factors preclude cure? Surgery, 2018. 163(6): p. 1238-1244.

24. Simmonds, P.C., J.N. Primrose, J.L. Colquitt, et al., Surgical resection of hepatic metastases from colorectal cancer: a systematic review of published studies. Br J Cancer, 2006. 94(7): p. 982-999.

25. Cummings, L.C., J.D. Payes and G.S. Cooper, Survival after hepatic resection in metastatic colorectal cancer: a population-based study. Cancer, 2007. 109(4): p. 718-726.

26. Rees, M., P.P. Tekkis, F.K. Welsh, et al., Evaluation of long-term survival after hepatic resection for metastatic colorectal cancer: a multifactorial model of 929 patients. Ann Surg, 2008. 247(1): p. 125135.

27. Tanis, E., B. Nordlinger, M. Mauer, et al., Local recurrence rates after radiofrequency ablation or resection of colorectal liver metastases. Analysis of the European Organisation for Research and Treatment of Cancer \#40004 and \#40983. Eur J Cancer, 2014. 50(5): p. 912-919.

28. Wong, S.L., P.B. Mangu, M.A. Choti, et al., American Society of Clinical Oncology 2009 Clinical Evidence Review on Radiofrequency Ablation of Hepatic Metastases From Colorectal Cancer. Journal of Clinical Oncology, 2010. 28(3): p. 493-508.

29. Beermann, M., J. Lindeberg, J. Engstrand, et al., 1000 consecutive ablation sessions in the era of computer assisted image guidance - Lessons learned. European journal of radiology open, 2018. 6: p. 1-8.

30. Shady, W., E.N. Petre, M. Gonen, et al., Percutaneous Radiofrequency Ablation of Colorectal Cancer Liver Metastases: Factors Affecting Outcomes-A 10-year Experience at a Single Center. Radiology, 2016. 278(2): p. 601-611.

31. Gillams, A., N. Goldberg, M. Ahmed, et al., Thermal ablation of colorectal liver metastases: a position paper by an international panel of ablation experts, the interventional oncology sans frontières meeting 2013. 2015. 25(12): p. 3438-3454.

32. White, R.R., I. Avital, C.T. Sofocleous, et al., Rates and patterns of recurrence for percutaneous radiofrequency ablation and open wedge resection for solitary colorectal liver metastasis. J Gastrointest Surg, 2007. 11(3): p. 256-263.

33. Lee, W.S., S.H. Yun, H.K. Chun, et al., Clinical outcomes of hepatic resection and radiofrequency ablation in patients with solitary colorectal liver metastasis. J Clin Gastroenterol, 2008. 42(8): p. 945949.

34. Otto, G., C. Duber, M. Hoppe-Lotichius, et al., Radiofrequency ablation as first-line treatment in patients with early colorectal liver metastases amenable to surgery. Ann Surg, 2010. 251(5): p. 796803.

35. Lawrence, T.S., J.M. Robertson, M.S. Anscher, et al., Hepatic toxicity resulting from cancer treatment. Int J Radiat Oncol Biol Phys, 1995. 31(5): p. 1237-1248.

36. Rusthoven, K.E., B.D. Kavanagh, H. Cardenes, et al., Multi-institutional phase I/II trial of stereotactic body radiation therapy for liver metastases. J Clin Oncol, 2009. 27(10): p. 1572-1578.

37. Rule, W., R. Timmerman, L. Tong, et al., Phase I dose-escalation study of stereotactic body radiotherapy in patients with hepatic metastases. Ann Surg Oncol, 2011. 18(4): p. 1081-1087.

38. Lee, M.T., J.J. Kim, R. Dinniwell, et al., Phase I study of individualized stereotactic body radiotherapy of liver metastases. J Clin Oncol, 2009. 27(10): p. 1585-1591.

39. Lykoudis, P.M., D. O'Reilly, K. Nastos, et al., Systematic review of surgical management of synchronous colorectal liver metastases. Br J Surg, 2014. 101(6): p. 605-612.

40. Feng, Q., Y. Wei, D. Zhu, et al., Timing of hepatectomy for resectable synchronous colorectal liver metastases: for whom simultaneous resection is more suitable--a meta-analysis. PLoS One, 2014. 9(8): p. e104348.

41. Kelly, M.E., G. Spolverato, G.N. Le, et al., Synchronous colorectal liver metastasis: a network meta- 
analysis review comparing classical, combined, and liver-first surgical strategies. J Surg Oncol, 2015. 111(3): p. 341-351.

42. Milinis, K., M. Thornton, A. Montazeri, et al., Adjuvant chemotherapy for rectal cancer: Is it needed? World journal of clinical oncology, 2015. 6(6): p. 225-236.

43. Folprecht, G., T. Gruenberger, W.O. Bechstein, et al., Tumour response and secondary resectability of colorectal liver metastases following neoadjuvant chemotherapy with cetuximab: the CELIM randomised phase 2 trial. Lancet Oncol, 2010. 11(1): p. 38-47.

44. Wong, R., D. Cunningham, Y. Barbachano, et al., A multicentre study of capecitabine, oxaliplatin plus bevacizumab as perioperative treatment of patients with poor-risk colorectal liver-only metastases not selected for upfront resection. Ann Oncol, 2011. 22(9): p. 2042-8.

45. Fusai, G. and B.R. Davidson, Strategies to increase the resectability of liver metastases from colorectal cancer. Dig Surg, 2003. 20(6): p. 481-496.

46. Huiskens, J., T.M. van Gulik, K.P. van Lienden, et al., Treatment strategies in colorectal cancer patients with initially unresectable liver-only metastases, a study protocol of the randomised phase 3 CAIRO5 study of the Dutch Colorectal Cancer Group (DCCG). BMC Cancer, 2015. 15: p. 365.

47. Hofheinz, R.D., F. Wenz, S. Post, et al., Chemoradiotherapy with capecitabine versus fluorouracil for locally advanced rectal cancer: a randomised, multicentre, non-inferiority, phase 3 trial. Lancet Oncol, 2012. 13(6): p. 579-588.

48. van Dijk, T.H., K. Tamas, J.C. Beukema, et al., Evaluation of short-course radiotherapy followed by neoadjuvant bevacizumab, capecitabine, and oxaliplatin and subsequent radical surgical treatment in primary stage IV rectal cancer. Ann Oncol, 2013. 24(7): p. 1762-1769.

49. Bisschop, C., T.H. van Dijk, J.C. Beukema, et al., Short-Course Radiotherapy Followed by Neoadjuvant Bevacizumab, Capecitabine, and Oxaliplatin and Subsequent Radical Treatment in Primary Stage IV Rectal Cancer: Long-Term Results of a Phase II Study. Ann Surg Oncol, 2017. 24(9): p. 2632-2638.

50. Verhoef, C., A.E.M. van der Pool, J.J. Nuyttens, et al., The "Liver-First Approach" for Patients with Locally Advanced Rectal Cancer and Synchronous Liver Metastases. Dis Colon Rectum. 2009. 52(1): p. 23-30.

51. Ayez, N., J.W.A. Burger, A.E. van der Pool, et al., Long-term Results of the "Liver First" Approach in Patients With Locally Advanced Rectal Cancer and Synchronous Liver Metastases. Dis Colon Rectum, 2013. 56(3): p. 281-287.

52. Organisation., W.H. WHO Definition of palliative care. 2015 27-01-2020]; Available from: https:// www.who.int/cancer/palliative/definition/en/.

53. Elferink, M.A., L.N. van Steenbergen, P. Krijnen, et al., Marked improvements in survival of patients with rectal cancer in the Netherlands following changes in therapy, 1989-2006. Eur J Cancer, 2010. 46(8): p. 1421-1429.

54. Cameron, M.G., C. Kersten, I. Vistad, et al., Palliative pelvic radiotherapy of symptomatic incurable rectal cancer-a systematic review. Acta Oncol, 2014. 53(2): p. 164-173.

55. Scoggins, C.R., I.M. Meszoely, C.D. Blanke, et al., Nonoperative management of primary colorectal cancer in patients with stage IV disease. Ann Surg Oncol, 1999. 6(7): p. 651-657.

56. Tebbutt, N.C., A.R. Norman, D. Cunningham, et al., Intestinal complications after chemotherapy for patients with unresected primary colorectal cancer and synchronous metastases. Gut, 2003. 52(4): p. 568-573.

57. Benoist, S., K. Pautrat, E. Mitry, et al., Treatment strategy for patients with colorectal cancer and synchronous irresectable liver metastases. Br J Surg, 2005. 92(9): p. 1155-1160.

58. Poultsides, G.A., E.L. Servais, L.B. Saltz, et al., Outcome of primary tumor in patients with synchronous stage IV colorectal cancer receiving combination chemotherapy without surgery as initial treatment. J Clin Oncol, 2009. 27(20): p. 3379-3384.

59. Gresham, G., D.J. Renouf, M. Chan, et al., Association between palliative resection of the primary tumor and overall survival in a population-based cohort of metastatic colorectal cancer patients. Ann Surg Oncol, 2014. 21(12): p. 3917-3923. 
60. Bajwa, A., N. Blunt, S. Vyas, et al., Primary tumour resection and survival in the palliative management of metastatic colorectal cancer. European Journal of Surgical Oncology, 2009. 35(2): p. 164-167.

61. Ferrand, F., D. Malka, A. Bourredjem, et al., Impact of primary tumour resection on survival of patients with colorectal cancer and synchronous metastases treated by chemotherapy: results from the multicenter, randomised trial Federation Francophone de Cancerologie Digestive 9601. Eur J Cancer, 2013. 49(1): p. 90-97.

62. Karoui, M., F. Roudot-Thoraval, F. Mesli, et al., Primary colectomy in patients with stage IV colon cancer and unresectable distant metastases improves overall survival: results of a multicentric study. Dis Colon Rectum, 2011. 54(8): p. 930-938.

63. Tanoue, Y., N. Tanaka and Y. Nomura, Primary site resection is superior for incurable metastatic colorectal cancer. World journal of gastroenterology, 2010. 16(28): p. 3561-3566.

64. Kim, M.S., M. Chung, J.B. Ahn, et al., Clinical significance of primary tumor resection in colorectal cancer patients with synchronous unresectable metastasis. J Surg Oncol, 2014. 110(2): p. 214-221.

65. Venderbosch, S., J.H. de Wilt, S. Teerenstra, et al., Prognostic value of resection of primary tumor in patients with stage IV colorectal cancer: retrospective analysis of two randomized studies and a review of the literature. Ann Surg Oncol, 2011. 18(12): p. 3252-3260.

66. Ahmed, S., A. Leis, A. Fields, et al., Survival impact of surgical resection of primary tumor in patients with stage IV colorectal cancer: results from a large population-based cohort study. Cancer, 2014. 120(5): p. 683-691.

67. Brouwer, N.P.M., A.C.R.K. Bos, V.E.P.P. Lemmens, et al., An overview of 25 years of incidence, treatment and outcome of colorectal cancer patients. International journal of cancer, 2018. 143(11): p. 27582766.

68. Bonjer, H.J., C.L. Deijen, G.A. Abis, et al., A Randomized Trial of Laparoscopic versus Open Surgery for Rectal Cancer. New England J of Medicine, 372(14): p. 1324-1332.

69. Jayne, D.G., P.J. Guillou, H. Thorpe, et al., Randomized trial of laparoscopic-assisted resection of colorectal carcinoma: 3-year results of the UK MRC CLASICC Trial Group. 2007. 25(21): p. 3061-3068.

70. Komenaka, I.K., K. Giffard, J. Miller, et al., COLOR: a randomized clinical trial comparing laparoscopic and open resection for colon cancer. Dig Surg, 2000. 17(6): p. 617-622.

71. Rickles, A.S., D.W. Dietz, G.J. Chang, et al., High Rate of Positive Circumferential Resection Margins Following Rectal Cancer Surgery: A Call to Action. Ann Surg, 2015. 262(6): p. 891-898.

72. Calin, M.A., S.V. Parasca, D. Savastru, et al., Hyperspectral imaging in the medical field: present and future. Applied Spectroscopy Reviews, 2014. 49(6): p. 435-447.

73. Nagaya, T., Y.A. Nakamura, P.L. Choyke, et al., Fluorescence-Guided Surgery. Frontiers in oncology, 2017. 7: p. 314-314.

74. Nguyen, Q.T. and R.Y.J.N.r.c. Tsien, Fluorescence-guided surgery with live molecular navigation-a new cutting edge. Nat Rev Cancer, 2013. 13(9): p. 653.

75. Keereweer, S., J.D.F. Kerrebijn, P.B.A.A. van Driel, et al., Optical Image-guided Surgery-Where Do We Stand? Mol Imaging Biol, 2011. 13(2): p. 199-207.

76. Miner, R.C., Image-Guided Neurosurgery. Journal of Medical Imaging and Radiation Sciences, 2017. 48(4): p. 328-335.

77. Schulz, C., S. Waldeck and U.M. Mauer, Intraoperative image guidance in neurosurgery: development, current indications, and future trends. Radiology research and practice, 2012. 2012: p. 197364-197364.

78. Cleary, K. and T.M. Peters, Image-Guided Interventions:Technology Review and Clinical Applications. 2010. 12(1): p. 119-142.

79. Goetz, A.F.H., Three decades of hyperspectral remote sensing of the Earth: A personal view. Remote Sensing of Environment, 2009. 113: p. S5-S16.

80. Edelman, G.J., T.G. van Leeuwen and M.C. Aalders, Visualization of latent blood stains using visible reflectance hyperspectral imaging and chemometrics. J Forensic Sci, 2015. 60 Suppl 1: p. S188-92.

81. Mahlein, A.K., M.T. Kuska, J. Behmann, et al., Hyperspectral Sensors and Imaging Technologies in 
Phytopathology: State of the Art. Annu Rev Phytopathol, 2018. 56: p. 535-558.

82. Liang, H., Advances in multispectral and hyperspectral imaging for archaeology and art conservation. Applied Physics A, 2012. 106(2): p. 309-323.

83. Zuzak, K.J., M.D. Schaeberle, E.N. Lewis, et al., Visible reflectance hyperspectral imaging: characterization of a noninvasive, in vivo system for determining tissue perfusion. Anal Chem, 2002. 74(9): p. 2021-8.

84. Randeberg, L.L., E.L. Larsen and L.O. Svaasand, Characterization of vascular structures and skin bruises using hyperspectral imaging, image analysis and diffusion theory. J Biophotonics, 2010. 3(1-2): p. 53-65.

85. Hattery, D., M. Hassan, S. Demos, et al. Hyperspectral imaging of Kaposi's Sarcoma for disease assessment and treatment monitoring. in Applied Imagery Pattern Recognition Workshop, 2002. Proceedings. 2002.

86. Liu, Z., H. Wang and Q.J.S. Li, Tongue tumor detection in medical hyperspectral images. Sensors, 2012. 12(1): p. 162-174.

87. Akbari, H., K. Uto, Y. Kosugi, et al., Cancer detection using infrared hyperspectral imaging. Cancer Sci, 2011. 102(4): p. 852-857.

88. Han, Z., A. Zhang, X. Wang, et al., In vivo use of hyperspectral imaging to develop a noncontact endoscopic diagnosis support system for malignant colorectal tumors. J Biomed Opt, 2016. 21(1): p. 16001.

89. Lu, G., J.V. Little, X. Wang, et al., Detection of head and neck cancer in surgical specimens using quantitative hyperspectral imaging. Clin Cancer Res, 2017. 23(18): p. 5426-5436.

90. Kho, E., L.L. De Boer, K.K. Van de Vijver, et al., Hyperspectral imaging for resection margin assessment during cancer surgery. Clin Cancer Res, 2019. 25(12): p. 3572-3580.

91. Han, Z., A. Zhang, X. Wang, et al., In vivo use of hyperspectral imaging to develop a noncontact endoscopic diagnosis support system for malignant colorectal tumors. J Biomed Opt, 2016. 21(1): p. 016001.

92. Rathore, S., M. Hussain, A. Ali, et al., A recent survey on colon cancer detection techniques. IEEE/ACM Trans Comput Biol Bioinform, 2013. 10(3): p. 545-563.

93. Kumashiro, R., K. Konishi, T. Chiba, et al., Integrated Endoscopic System Based on Optical Imaging and Hyperspectral Data Analysis for Colorectal Cancer Detection. Anticancer Res, 2016. 36(8): p. 3925-3932.

94. Senft, C., A. Bink, K. Franz, et al., Intraoperative MRI guidance and extent of resection in glioma surgery: a randomised, controlled trial. The Lancet Oncology, 2011. 12(11): p. 997-1003.

95. Tormenti, M.J., D.B. Kostov, P.A. Gardner, et al., Intraoperative computed tomography imageguided navigation for posterior thoracolumbar spinal instrumentation in spinal deformity surgery. Neurosurg Focus, 2010. 28(3): p. E11.

96. Aschendorff, A., W. Maier, K. Jaekel, et al., Radiologically assisted navigation in cochlear implantation for X-linked deafness malformation. Cochlear Implants Int, 2009. 10 Suppl 1: p. 14-18.

97. Nijkamp, J., K.F.D. Kuhlmann, O. Ivashchenko, et al., Prospective study on image-guided navigation surgery for pelvic malignancies. J Surg Oncol, 2019. 119(4): p. 510-517. 



\section{Chapter 2}

\section{Multicentre study of short-course radiotherapy, systemic therapy and resection/ablation for stage IV rectal cancer}

Esther N.D. Kok Klaas Havenga Pieter J. Tanis Johannes H.W. de Wilt Jeroen Hagendoorn Femke P. Peters Jeroen Buijsen

Harm J.T. Rutten Koert F.D. Kuhlmann The Dutch Stage IV Rectal Cancer Group 


\section{Abstract}

Background: The optimal treatment sequence for patients with rectal cancer and synchronous liver metastases remains unclear. The aim of this study was to evaluate the feasibility and effectiveness of short-course pelvic radiotherapy $(5 \times 5 \mathrm{~Gy})$, followed by systemic therapy and local treatment of all tumour sites in patients with potentially curable stage IV rectal cancer in daily practice.

Methods: This was a retrospective study performed in eight tertiary referral centres in the Netherlands. Patients aged 18 years or above with rectal cancer and potentially resectable liver \pm extrahepatic metastases, treated between 2010 and 2015, were eligible. Main outcomes included full completion of treatment schedule, symptom control and survival.

Results: In total, 169 patients were included with a median follow-up of 50 months (range 2 - 89). The completion rate for the entire treatment schedule was $65.7 \%$. Three-year progression-free survival and overall survival (OS) rates were $24.2 \%$ (95\% Cl: 16.6--31.6) and 8.8\% (40.4-57.2), respectively. Median OS of patients who responded well and completed the treatment schedule was 51.5 months, compared with 15.1 months for patients who did not complete the treatment $(p<0.001)$. Adequate symptom control of the primary tumour was achieved in $87.0 \%$ of all patients.

Conclusion: Multimodal treatment leads to relief of symptoms in most patients, and is associated with good survival rates in those able to complete the schedule. 


\section{Introduction}

Curative treatment of stage IV rectal cancer is challenging and up to $80 \%$ of the patients have unresectable liver metastases at diagnosis [1]. Previous studies have shown that systemic therapy can downsize the metastases with subsequent conversion to resectable or ablatable disease $[2,3]$. Nevertheless, radical resection of multiple tumour sites remains challenging in patients with metastatic disease [4]. In the setting of synchronous metastases, the rectal tumour is often locally advanced. Traditionally, the preferred treatment is long-course chemoradiotherapy including 5-fluorouracil or capecitabine as radiation sensitizer, with limited effect on distant metastases $[5,6]$. Consequently, the metastatic disease is left untreated for a significant period of time.

To minimize treatment delay, a new treatment schedule was introduced that combined radiotherapy of the primary tumour with a timely and adequate dose of systemic therapy to address the distant disease. This schedule consisted of preoperative short-course radiotherapy $(5 \times 5 \mathrm{~Gy})$, followed by six cycles of capecitabine-oxaliplatinbevacizumab (CAPOX-B), with the intention of subsequent surgical resection or ablation of all tumour sites [7]. In a phase II single-arm study, 50 patients with rectal cancer and resectable or ablatable liver and/or lung metastases underwent this treatment schedule [7]. Eventually, 36 patients (72\%) underwent radical surgical treatment with 2 -year recurrence-free and overall survival rates of $64 \%$ and $80 \%$, respectively.

After completion of this so-called M1 study, multiple centres in the Netherlands implemented this treatment schedule in routine daily practice, as it effectively combines local control of the rectal tumour with a timely start of systemic therapy. Although the original study only included patients with treatable and limited metastatic disease, less strict eligibility criteria are used in daily practice. The aim of the present study was to evaluate the feasibility and effectiveness of this treatment schedule in stage IV rectal cancer patients in daily practice.

\section{Methods}

This was a retrospective study in eight centres in the Netherlands: Amsterdam University Medical Centre, Catharina Hospital Eindhoven, University Medical Centre Groningen, Leiden University Medical Centre, Maastricht University Medical Centre, Netherlands Cancer Institute (Amsterdam), Radboud University Medical Centre (Nijmegen) and University Medical Centre Utrecht. Patients with a diagnosis of synchronous metastasized rectal cancer were identified from institutional databases and registrations between January 2010 and December 2015. 
Patients were eligible if rectal cancer with (potentially) curable liver \pm extrahepatic metastases was confirmed histologically and they were scheduled for the multimodality treatment, consisting of $5 \times 5$ Gy pelvic radiotherapy followed by systemic therapy and subsequent surgery, with or without other local treatment modalities. Simultaneous lung or distant lymph node metastases were accepted as long as treatment with curative intent was deemed possible after systemic therapy. Standard diagnostic investigation consisted of pelvic MRI for local staging of the rectal tumour and CT of the chest, abdomen and pelvis to detect distant metastases. All patients were discussed in a multidisciplinary meeting with (intervention) radiologists, and medical, radiation and surgical oncologists. Clinical data of eligible patients were collected from medical records and anonymized. This study was approved by the institutional review board (METC17.1695/M17CRM).

\section{Neoadjuvant treatment and reassessment}

Patients with significant signs of obstruction at diagnosis received a diverting stoma. Neoadjuvant short-course radiotherapy $(5 \times 5 \mathrm{~Gy})$ was given to the primary tumour, mesorectum and regional lymph nodes. Systemic therapy started approximately 2 weeks after the last fraction of radiation. The choice of systemic therapy regimen was at the discretion of the local team. After three cycles of systemic therapy, radiological evaluation of response was performed using CT and MRI. In each participating centre a multidisciplinary team decided on the next optimal treatment step: continuation of systemic treatment, staged or simultaneous local treatment of primary tumour and/or distant metastases, or palliative treatment.

\section{Surgery and histopathology}

After completion of neoadjuvant treatment, the multidisciplinary team reviewed the resectability of the primary tumour and metastases. The optimal sequence of surgical treatment and type of surgical procedure with or without other local treatment modalities were tailored to the individual patient. Other local treatment modalities included radiofrequency ablation (RFA) and stereotactic radiotherapy. Primary tumour resection was performed by partial or total mesorectal excision [8]. The choice of surgical procedure and the use of a diverting stoma was at the discretion of the local surgeon. Treatment of extrahepatic metastases included pulmonary metastasectomy, pulmonary RFA, stereotactic radiotherapy of the lung and lymph node resection. In patients with complete response of the primary tumour and/or the metastases to neoadjuvant treatment, a watch-and-wait approach was sometimes chosen [9]. After surgical resection of all tumour sites, no adjuvant therapy was given. 
Histopathological evaluation of the specimen was performed by the local pathologist. Downstaging of the primary tumour was evaluated, comparing the baseline CT status with the (y)pT status. A positive resection margin (R1) of the liver was defined as any infiltration of tumour cells in the resection margin. After rectal surgery, a positive resection margin was defined by presence of tumour cells $\leq 1 \mathrm{~mm}$ of the circumferential resection margin.

\section{Outcomes}

The main outcomes were completion of the entire treatment schedule, pathological response and overall survival (OS). Ancillary outcomes were treatment-related toxicity, surgical complications, symptom control of the primary tumour and progression-free survival (PFS). Completion of the treatment schedule was defined as neoadjuvant short-course radiotherapy, at least two cycles of systemic therapy and subsequent local treatment of all tumour sites. Treatment-related toxicity was registered using the US National Cancer Institute Common Terminology Criteria for Adverse Events version 4.0 [10]. Radiotherapy-related toxicity was classified as acute and late toxicity, defined as toxicities within or after 3 months. Surgical complications for both liver and rectal surgery were graded using the Clavien-Dindo classification [11]. Tumour response evaluation was assessed using the RECIST (Response Evaluation Criteria In Solid Tumours) criteria version 1.0 [12]. PFS was calculated from the first day of radiotherapy to the first evidence of recurrence (local, regional or metastatic) or until death. OS was calculated from the first day of radiotherapy until death or last documented follow-up.

\section{Statistical analysis}

All analyses were performed using SPSS ${ }^{\circledR}$ version 24.0 (IBM, Armonk, New York, USA). Patients and treatment characteristics are presented as percentages, medians with ranges or medians with $95 \% \mathrm{Cl}$. Categorical variables were compared using the $\mathrm{X}^{2}$ test, and continuous variables with the Mann-Whitney $U$ test. OS and PFS were calculated using Kaplan-Meier curves. The association of completing the treatment schedule with OS was analysed using an extended Cox proportional hazards model, in which treatment completion was used as the time-dependent co-variable to avoid immortal time bias. The log rank test was used to compare survival probabilities between subgroups. Univariable and multivariable Cox regression analyses were performed to identify predictors of survival. A binary logistic regression model was used to identify prognostic factors of treatment completion. All variables with significance of $P \leq 0.20$ in univariable analysis were entered in the multivariable analysis. $\mathrm{P}<0.05$ (two-sided) was regarded as statistically significant. 
Table 1. Patient characteristics

\begin{tabular}{|c|c|}
\hline Characteristics & $\begin{array}{c}\text { No. of patients } \\
n=169\end{array}$ \\
\hline Age at start of treatment (years) & $63(32-83)$ \\
\hline Sex ratio $(M: F)$ & $109: 60$ \\
\hline $\begin{array}{l}\text { WHO performance status } \\
\qquad \begin{array}{l}0 \\
1 \\
2\end{array}\end{array}$ & $\begin{array}{c}128(75.7) \\
33(19.5) \\
8(4.8)\end{array}$ \\
\hline $\begin{array}{l}\text { Clinical tumor and nodal stage } \\
\text { T2N0 } \\
\text { T2N1-2 } \\
\text { T3N0 } \\
\text { T3N1-2 } \\
\text { T4N1-2 }\end{array}$ & $\begin{array}{c}2(1.2) \\
11(6.5) \\
10(5.9) \\
117(69.2) \\
29(17.2)\end{array}$ \\
\hline Mesorectal fascia involvement & $75(44.4)$ \\
\hline $\begin{array}{l}\text { Location of primary rectal cancer } \\
\qquad \begin{array}{l}\text { Low }(0-5 \mathrm{~cm}) \\
\text { Middle }(5-10 \mathrm{~cm}) \\
\text { High }(10-15 \mathrm{~cm}) \\
\text { Unknown }\end{array}\end{array}$ & $\begin{array}{l}67(39.6) \\
76(45.0) \\
24(14.2) \\
2(1.2)\end{array}$ \\
\hline $\begin{array}{l}\text { Metastatic site } \\
\text { Liver only } \\
\text { Liver and lung } \\
\text { Liver and distant lymph nodes } \\
\text { Liver, lung and distant lymph nodes }\end{array}$ & $\begin{array}{l}130(76.9) \\
19(11.3) \\
19(11.3) \\
1(0.6)\end{array}$ \\
\hline Number of liver metastases & $3(1-20)$ \\
\hline $\begin{array}{c}\text { Location of liver metastases } \\
\text { Unilobar } \\
\text { Bilobar }\end{array}$ & $\begin{array}{l}95(56.2) \\
74(43.8)\end{array}$ \\
\hline Diameter largest metastasis (cm) & $3.0(0.8-22.1)$ \\
\hline
\end{tabular}

Data are reported as number (percent) or median (range)

\section{Results}

Between January 2010 and December 2015, 169 patients with potentially resectable or ablatable stage IV rectal cancer fulfilled the criteria and started the treatment schedule. Patient and treatment characteristics are shown in Tables 1 and 2, respectively. In 70 patients (41.4\%), all tumour sites were considered surgically amenable for local treatment before any neoadjuvant treatment. Neoadjuvant $5 \times 5$ Gy radiotherapy was completed in 168 patients; the other patient received only four fractions of $5 \mathrm{~Gy}$ owing to bowel toxicity (perforation) (Figure 1). Overall, 159 of all patients (94.1\%) received between three and eight cycles of systemic therapy. Systemic therapy consisted of capecitabine and oxaliplatin (CAPOX); capecitabine, oxaliplatin and bevacizumab (CAPOX-B); capecitabine and bevacizumab (CAP-B); fluorouracil, oxaliplatin and folinic acid (FOLFOX), fluorouracil, oxaliplatin, folinic acid and bevacizumab (FOLFOX-B) or capecitabine monotherapy. Median follow-up was 49.5 months ( $95 \% \mathrm{Cl}$ : 43.6 - 55.6). 
Table 2. Treatment characteristics

\begin{tabular}{|c|c|}
\hline Characteristics & $\begin{array}{l}\text { No. of patients } \\
n=169\end{array}$ \\
\hline $\begin{array}{l}\text { Diverting stoma } \\
\text { Before all treatment } \\
\text { During neoadjuvant treatment }\end{array}$ & $\begin{array}{c}26(15.4) \\
14(8.3)\end{array}$ \\
\hline $\begin{array}{l}\text { Radiotherapy } \\
5 \times 5 \text { Gy } \\
4 \times 5 \text { Gy }\end{array}$ & $\begin{array}{l}168(99.4) \\
1(0.6)\end{array}$ \\
\hline $\begin{array}{l}\text { Chemotherapy } \\
\text { CAPOX } \\
\text { CAPOX-B } \\
\text { FOLFOX } \\
\text { FOLFOX-B } \\
\text { CAP } \\
\text { CAP-B }\end{array}$ & $\begin{array}{c}n=165 \\
41(24.9) \\
109(66.1) \\
5(3.0) \\
5(3.0) \\
4(2.4) \\
1(0.6)\end{array}$ \\
\hline $\begin{array}{l}\text { Total administered cycles } \\
\qquad \begin{array}{l}1-3 \text { cycles } \\
4-6 \text { cycles } \\
\geq 7 \text { cycles }\end{array}\end{array}$ & $\begin{array}{l}6(1-14) \\
48(29.1) \\
102(61.8) \\
15(9.1)\end{array}$ \\
\hline $\begin{array}{l}\text { Rectum resection }{ }^{1} \\
\text { Low Anterior Resection } \\
\text { Hartmann procedure } \\
\text { Abdominoperineal resection }\end{array}$ & $\begin{array}{l}n=112 \\
74(66.1) \\
13(11.6) \\
25(22.3)\end{array}$ \\
\hline $\begin{array}{l}\text { Liver treatment } \\
\text { Liver resection } \\
\text { RFA alone } \\
\text { Resection + RFA } \\
\text { Radiation therapy }\end{array}$ & $\begin{array}{c}n=132 \\
83(62.9) \\
13(9.8) \\
34(25.8) \\
2(1.5)\end{array}$ \\
\hline $\begin{array}{l}\text { Extrahepatic metastases treatment } \\
\text { Lung: }\end{array}$ & \\
\hline $\begin{array}{l}\text { Metastasectomy } \\
\text { RFA } \\
\text { Radiation therapy } \\
\text { Lymph node resection } \\
\text { Combination lymph node and rectum resection } \\
\text { Rectal radiation therapy expanded for lymph node }\end{array}$ & $\begin{array}{l}6(3.6) \\
3(1.8) \\
2(1.2) \\
2(1.2) \\
4(2.4) \\
2(1.2)\end{array}$ \\
\hline $\begin{array}{l}\text { Hospital stay in days } \\
\text { Rectum resection } \\
\text { Liver resection } \\
\text { Simultaneous resection }\end{array}$ & $\begin{array}{l}8(3-29) \\
7(1-62) \\
14(5-57)\end{array}$ \\
\hline
\end{tabular}

Data are reported as number (percent) or median (range)

'Includes 36 local treatment procedures performed during simultaneous resections

Abbreviations: CAP: capecitabine, OX: oxaliplatin, B: bevacizumab, FOLFOX: 5-FU \& folinic acid with oxaliplatin; RFA:

Radiofrequency ablation

\section{Completion of entire treatment schedule}

In total, 111 patients (65.7\%) completed the entire treatment schedule. After neoadjuvant treatment, 88 patients received liver-first approach, and in 17 primary tumour resection was performed first. Simultaneous surgery of the primary tumour and liver metastases was performed in 36 patients. A complete response at all tumour sites after neoadjuvant treatment was seen in one patient, and a watch-and-wait approach 
Chapter 2

Figure 1. Flow diagram for all patients in the study

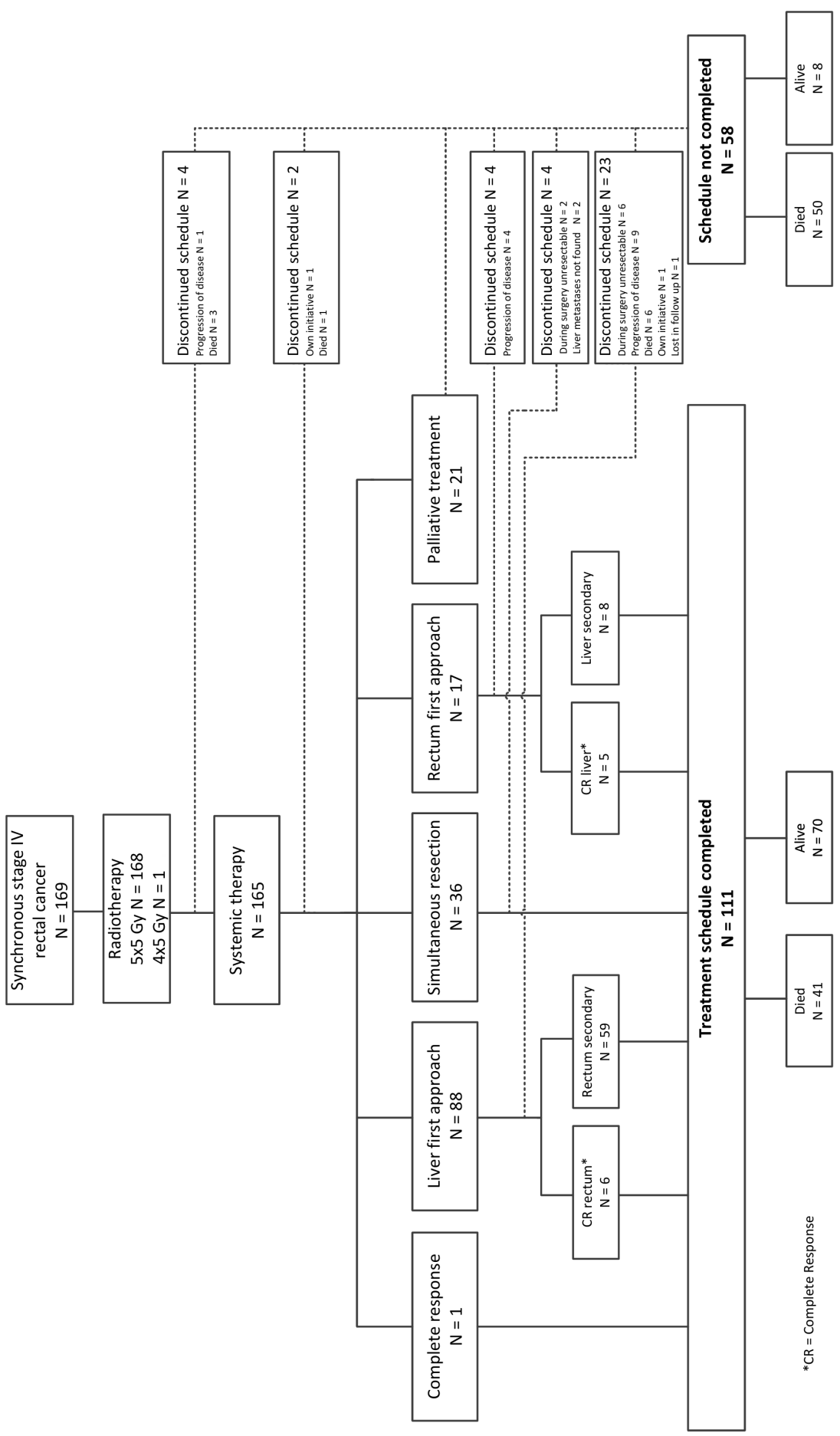


was aplied (Figure 1).

In multivariable logistic regression, the number of liver metastases (HR: 0.82; 95\% Cl: $0.66-0.94 ; \mathrm{P}=0.004$ ) was the only significant predictor for completion of the entire treatment schedule. Of the 58 patients who were not able to complete the treatment schedule, 24 (41\%) received palliative systemic treatment, 19 (33\%) refrained from active treatment, four (7\%) received palliative radiotherapy, ten (17\%) died and one (2\%) was lost in follow up.

\section{Progression-free and overall survival}

Forty-eight patients had progressive disease during treatment and 69 after completing the entire treatment schedule. Progression of disease was observed most frequently in the liver (Table 3). Seven patients developed local recurrence after rectal surgery. Of the 48 patients who showed progression during the treatment schedule, seven were able to complete the entire schedule and 41 were not. The 1-, 3- and 5-year PFS rates for all patients were $59.3 \%$ (95\% Cl: 51.6 - 66.9), 24.2\% (16.6 - 31.6) and 17.1\% (9.6 - 24.5), respectively. Median OS for all patients was 35.7 (range 1.0 - 88.4) months. The 1-, 3and 5-year OS rates were $85.2 \%$ (95\% Cl: 79.7 - 90.7), 48.8\% (40.4 - 57.2) and $31.1 \%$ (22.8 - 39.9), respectively (Figure 2). The median OS for the 111 patients who completed the entire treatment schedule was 51.5 months, compared with 15.1 months in patients who did not complete the treatment $(P<0.001)$. The 3- and 5-year OS rates for patients who completed the entire treatment schedule were $73.6 \%$ (64.1 - 83.1) and $46.8 \%(34.8$ - 58.7), respectively.

In multivariable analysis, the number of liver metastases (HR: 1.11;95\% Cl: $1.02-1.20 ; \mathrm{P}$ $=0.011$ ) was associated with decreased OS (Table 4).

Table 3. Anatomic location of progression of disease; divided in progression during the treatment schedule and after completing the entire treatment schedule

\begin{tabular}{lcc}
\hline Anatomic location & During treatment schedule & After treatment schedule \\
\hline Liver & 25 & 23 \\
Lung & 3 & 13 \\
Liver and lung & 8 & 15 \\
Liver and rectum (local recurrence) & 3 & 2 \\
Lung and rectum (local recurrence) & 0 & 2 \\
Other & 9 & 14 \\
\hline
\end{tabular}




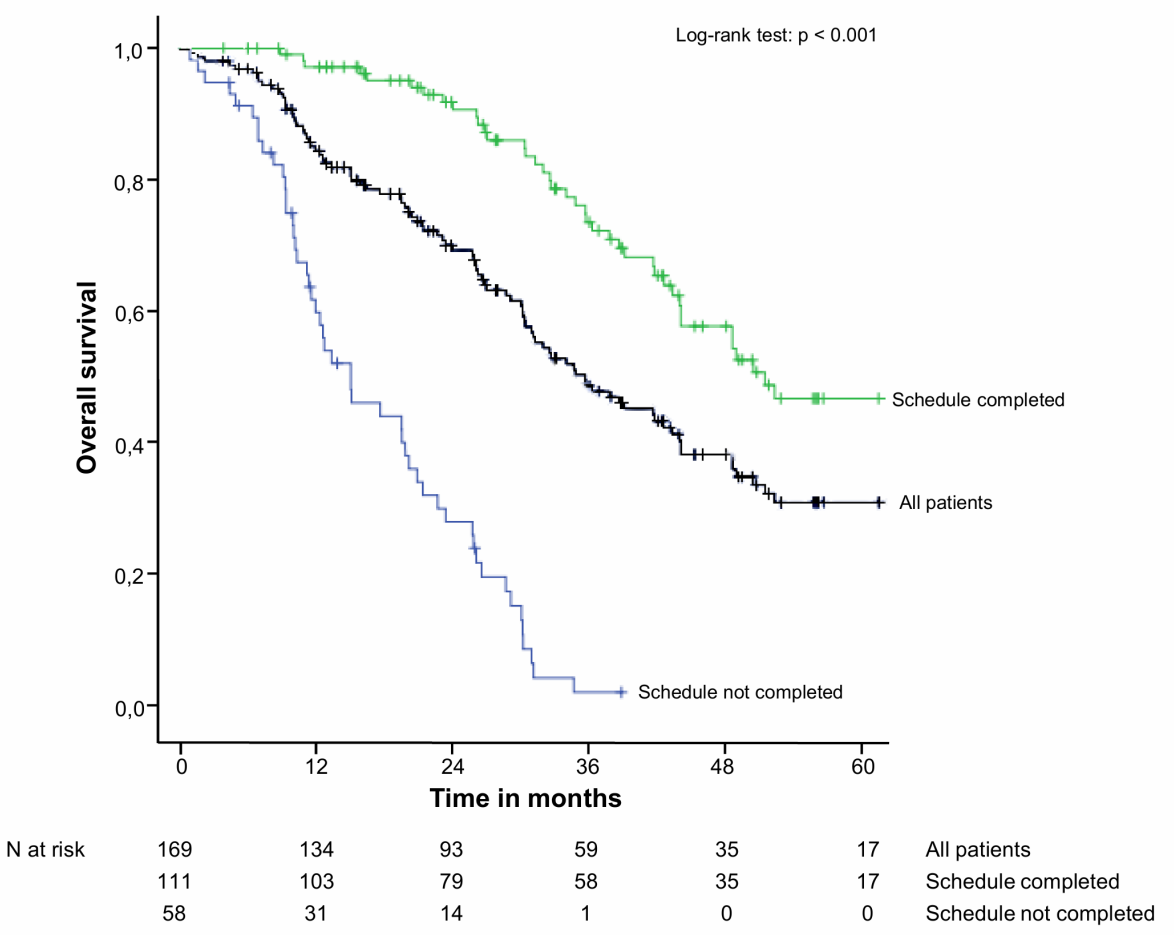

Figure 2. Kaplan-Meier analysis of overall survival for the whole cohort, patients who completed the treatment schedule, and those who did not

\section{Evaluation of radiological and pathological responses}

Complete radiological and endoscopic response of the rectal tumour was observed in 11 of 165 patients (6.7\%), allowing a watch-and-wait approach. Five of these patients had recurrence of the rectal tumour within 6 months and underwent rectal resection. In six patients, no recurrence was observed during follow-up (median follow-up 26.6 months). A partial response was observed in $49.1 \%$ of patients. A radiological complete response of the liver was observed in five patients (3.0\%).

Clear pathological margins at rectal surgery (R0) were accomplished in 99 (88.4\%) of the 112 patients who had a rectal resection, including 14 patients (12.5\%) with a pathological complete response. Pathological downstaging of the primary tumour was seen in 38 patients (33.9\%). Resection margins of the liver specimen were involved (R1) in 11 (10.3\%) of the 107 patients undergoing surgical resection of liver metastatses. A pathological complete response of the liver was reported in 15 patients (14.0\%). 
Table 4. Univariable and multivariable analyses for overall survival

\begin{tabular}{lcccc}
\hline & \multicolumn{2}{c}{ Univariable analysis } & \multicolumn{2}{c}{ Multivariable analysis } \\
\cline { 2 - 5 } Variables & HR & P-value & HR & P-value \\
\hline Age at diagnosis (continuous) & $0.99(0.97-1.01)$ & 0.351 & & \\
Sex (M versus F) & $1.18(0.76-1.84)$ & 0.453 & & \\
WHO performance status (2 versus 0-1) & $1.06(0.72-1.55)$ & 0.782 & & 0.011 \\
No. of liver metastases & & & & 0.252 \\
$\quad$ Continuous & $1.12(1.04-1.21)$ & 0.003 & $1.11(1.02-1.20)$ & \\
$\quad$ > 4 versus $\leq 4$ & $1.76(1.13-2.76)$ & 0.013 & $1.30(0.83-2.03)$ & 0.379 \\
CT category (IV versus II-III) & $1.47(0.95-2.27)$ & 0.086 & & \\
CN category (II versus 0-I) & $1.23(0.86-1.75)$ & 0.253 & & \\
Extrahepatic disease (yes versus no) & $1.37(0.85-0.20)$ & 0.200 & $1.21(0.77-2.01)$ & 0.06 \\
\hline
\end{tabular}

\section{Toxicity and morbidity}

Grade III and IV radiotherapy-related toxicity was observed in 11 of the 169 patients (6.5\%) (Table 5). One patient died within 1 month after radiotherapy from rectal bleeding. Chemotherapy-related grade III and IV toxicity was observed in 45 patients (27.3\%). Gastrointestinal complaints and pulmonary embolism were reported most frequently.

Severe surgical complications (grade III-IV) were observed after rectal resection in 14 patients (12.5\%). The most frequent complications were ileus and anastomotic leakage. Grade III-IV complications after liver treatment occurred in 16 of 132 patients (13.7\%). Twelve of the 16 patients suffered from infection in the abdomen/thorax or of the wound. Within 30 days of liver surgery, three patients (2.3\%) died from acute coronary syndrome. After simultaneous resection, 16 of the 36 patients (44\%) experienced grade III-IV complications. The most common complications were abdominal, pulmonary and wound infection.

\section{Control of symptoms from the primary tumour}

Despite short-course radiotherapy, 35 patients experienced rectal complaints during the treatment schedule. The most reported complaints were pain, obstruction and rectal bleeding. Of all patients, $16(8.5 \%)$ received pharmacological treatment, 14 (8.2\%) required a diverting stoma during systemic treatment, and in four patients (2.4\%) rectal surgery was performed earlier than planned. In the remaining 131 patients $(79.4 \%)$, the treatment schedule provided local symptom control during follow-up in both curative and palliative settings. 
Table 5. Neoadjuvant treatment toxicity and local treatment complications

\begin{tabular}{|c|c|c|c|}
\hline & No. of events & $\begin{array}{c}\text { No. of } \\
\text { patients } \\
\text { with events }\end{array}$ & $\begin{array}{l}\text { Total no. of } \\
\text { patients }\end{array}$ \\
\hline \multicolumn{4}{|l|}{ Radiotherapy-related toxicity } \\
\hline $\begin{array}{l}\text { Grade } 3-4 \\
\text { Acute } \\
\text { Late }\end{array}$ & $\begin{array}{l}9 \\
2\end{array}$ & 11 & 169 \\
\hline $\begin{array}{l}\text { Grade } 5 \\
\text { Acute } \\
\text { Late }\end{array}$ & $\begin{array}{l}1 \\
0\end{array}$ & 1 & 169 \\
\hline \multicolumn{4}{|l|}{ Chemotherapy-related toxicity ${ }^{1}$} \\
\hline $\begin{array}{l}\text { Grade } 3-4 \\
\text { Gastrointestinal } \\
\text { Vascular } \\
\text { Neurologic } \\
\text { Dermatologic } \\
\text { Hematologic } \\
\text { Cardiologic } \\
\text { Infection }\end{array}$ & $\begin{array}{l}31 \\
12 \\
7 \\
7 \\
5 \\
2 \\
2\end{array}$ & 45 & 165 \\
\hline $\begin{array}{l}\text { Grade } 5 \\
\text { Unknown toxicity }\end{array}$ & & $\begin{array}{c}0 \\
22\end{array}$ & $\begin{array}{l}165 \\
165\end{array}$ \\
\hline \multicolumn{4}{|l|}{ Surgical complications } \\
\hline $\begin{array}{l}\text { Grade } 3 \text { - 4, staged rectum resection } \\
\text { Ileus / gastroparesis } \\
\text { Anastomotic leakage } \\
\text { Presacral abscess } \\
\text { Wound dehiscence } \\
\text { Other }\end{array}$ & $\begin{array}{l}3 \\
4 \\
4 \\
3 \\
2 \\
3\end{array}$ & 14 & 112 \\
\hline Grade 5 & & 0 & 112 \\
\hline $\begin{array}{l}\text { Grade } 3-4 \text {, staged liver treatment }{ }^{2} \\
\text { Infection / abscess } \\
\text { Abdominal } \\
\text { Thorax } \\
\text { Wound } \\
\text { Bleeding } \\
\text { Bile duct perforation or stenosis } \\
\text { Other }\end{array}$ & $\begin{array}{l}6 \\
4 \\
2 \\
3 \\
3 \\
4\end{array}$ & 16 & 132 \\
\hline $\begin{array}{l}\text { Grade } 5 \\
\quad \text { Acute coronary syndrome }\end{array}$ & 3 & 3 & 132 \\
\hline $\begin{array}{l}\text { Grade } 3-4 \text {, simultaneous resection }{ }^{2} \\
\text { Infection / abscess } \\
\text { Abdominal } \\
\text { Thorax } \\
\text { Wound } \\
\text { lleus / gastroparesis } \\
\text { Wound dehiscence } \\
\text { Other }\end{array}$ & $\begin{array}{l}6 \\
6 \\
3 \\
4 \\
3 \\
4\end{array}$ & 16 & 36 \\
\hline Grade 5 & & 0 & 36 \\
\hline
\end{tabular}

'Some patients had more than one toxicity; ${ }^{2}$ some patients had more than one complication. 


\section{Discussion}

This evaluation of clinical practice shows that subsequent local treatment of all tumour sites after multimodal neoadjuvant therapy was achieved in the majority of patients, with tolerable morbidity and mortality. The number of liver metastases at diagnosis appeared to be the only predictor of treatment completion and survival.

In the initial prospective $\mathrm{M} 1$ study, $80 \%$ of patients received local treatment at all tumour sites, with a 5 -year OS rate of $38 \%[7,13]$, whereas in the present study these numbers were lower. In the initial trial, only patients with limited metastatic disease, who were considered treatable with curative intent at baseline, were included. In the present study, less than half of the patients had resectable metastases at baseline and the resectability of the metastases inmost patients was highly dependent on the response of the systemic treatment.

In locally advanced rectal cancer, short-course radiotherapy with subsequent systemic therapy can be an alternative for long-course chemoradiotherapy. A large randomized study compared short-course radiotherapy combined with three cycles of FOLFOX4 with long-course chemoradiotherapy in patients with locally advanced rectal cancer (cT4 or fixed cT3) [14]. No significant difference was observed in local efficacy between the treatments. A different study with a similar design found improved pathological tumour downstaging and better disease-free and metastasis-free survival in patients treated with short-course radiotherapy plus chemotherapy [15]. In the present study, complete pathological response of the primary tumour was observed in $12.5 \%$, omitting surgical treatment in six patients, and a partial response in $49.1 \%$.

Symptom control is important in patients with rectal cancer. Short-course pelvic radiotherapy has been shown to be an effective palliative treatment modality in patients with symptomatic rectal cancer $[16,17]$. Many clinicians, however, are hesitant to use short-course radiotherapy in patients with locally advanced disease because of the perceived risk of providing less optimal local control compared with that obtained with chemoradiotherapy. In the present study, adequate symptomatic local control of the primary tumour was achieved in the majority of the patients. This confirms that short-course radiotherapy followed by systemic chemotherapy is an effective and safe treatment in stage IV rectal cancer, in both the curative and palliative setting.

Systemic chemotherapy plays a significant role in downstaging liver metastases and eradication of microscopic disease. Earlier studies showed that induction chemotherapy can convert initially unresectable liver metastases to resectable disease $[18,19]$. In the present study, 99 patients (58.6\%) had potentially or borderline resectable/ablatable liver metastases at the time of diagnosis. Of these patients, $60.1 \%$ had a partial response 
after systemic treatment, enabling curative liver treatment. This confirms that systemic treatment can downsize metastases to resectable disease, yet the optimal regimen has not been defined. Currently, a Dutch multicentre randomized phase 3 trial (CAIRO5) is underway that compares multiple systemic treatment regimens in patients with colorectal cancer and initially unresectable liver-only metastases (NCT02162563) [20]. The estimated study completion date is the end of 2020.

An alternative treatment for stage IV rectal cancer has been published previously [21-23]. Patients received chemotherapy followed by a liver-first approach. After liver surgery, (chemo)radiotherapy was administered with subsequent primary tumour resection. Ninety of the 129 patients (69.8\%) completed the protocol, of whom ten had a (near) complete response of the primary tumour. A disadvantage of this approach is that the duration of treatment is longer compared with the schedule in the present study. Moreover, with this approach a simultaneous resection is not feasible because of the treatment sequence.

Limitations of this study include the retrospective design with its inherent methodological shortcomings. Selection and allocation biases might have been introduced. The total number of patients with synchronous liver metastases of rectal cancer is not known. Whether the primary tumour and metastases were resectable was determined by the local multidisciplinary team, and definitions probably differed between centres. Finally, it remains unclear to which degree finishing the treatment schedule attributed to the survival difference between patients who completed the treatment and who did not as this may be a confounder for tumour biology. 


\section{References}

1. Kok E.N.D., Kobus C, Siemons S, Grootscholten C, Dewit L, van den Berg J, et al. Treatment of stage IV rectal cancer: A multicentre study on short-course radiotherapy followed by neoadjuvant systemic therapy and subsequent surgical treatment. European Journal of Surgical Oncology 2019;45(2): e9.

2. Adam R, Delvart V, Pascal G, Valeanu A, Castaing D, Azoulay D, et al. Rescue surgery for unresectable colorectal liver metastases downstaged by chemotherapy: a model to predict long-term survival. Annals of surgery 2004;240(4): 644.

3. Lam VWT, Spiro C, Laurence JM, Johnston E, Hollands MJ, Pleass HCC, et al. A Systematic Review of Clinical Response and Survival Outcomes of Downsizing Systemic Chemotherapy and Rescue Liver Surgery in Patients with Initially Unresectable Colorectal Liver Metastases. Annals of surgical oncology 2012;19(4): 1292-1301.

4. Devaud N, Kanji ZS, Dhani N, et al. Liver resection after chemotherapy and tumour downsizing in patients with initially unresectable colorectal cancer liver metastases. HPB (Oxford) 2014;16(5): 475480.

5. Reddy SK, Pawlik TM, Zorzi D, Gleisner AL, Ribero D, Assumpcao L, et al. Simultaneous resections of colorectal cancer and synchronous liver metastases: a multi-institutional analysis. Annals of surgical oncology 2007;14(12): 3481-3491.

6. Sauer R, Becker H, Hohenberger W, Rödel C, Wittekind C, Fietkau R, et al. Preoperative versus Postoperative Chemoradiotherapy for Rectal Cancer. New England Journal of Medicine 2004;351(17): 1731-1740.

7. Hofheinz RD, Wenz F, Post S, Matzdorff A, Laechelt S, Hartmann JT, et al. Chemoradiotherapy with capecitabine versus fluorouracil for locally advanced rectal cancer: a randomised, multicentre, noninferiority, phase 3 trial. The Lancet Oncology 2012;13(6): 579-588.

8. van Dijk TH, Tamas K, Beukema JC, Beets GL, Gelderblom AJ, de Jong KP, et al. Evaluation of shortcourse radiotherapy followed by neoadjuvant bevacizumab, capecitabine, and oxaliplatin and subsequent radical surgical treatment in primary stage IV rectal cancer. Annals of oncology : official journal of the European Society for Medical Oncology 2013;24(7): 1762-1769.

9. Glynne-Jones R, Wyrwicz L, Tiret E, Brown G, Rödel C, Cervantes A, et al. Rectal cancer: ESMO Clinical Practice Guidelines for diagnosis, treatment and follow-upt. Annals of Oncology 2017;28(suppl_4): iv22-iv40.

10. Renehan AG, Malcomson L, Emsley R, Gollins S, Maw A, Myint AS, et al. Watch-and-wait approach versus surgical resection after chemoradiotherapy for patients with rectal cancer (the OnCoRe project): a propensity-score matched cohort analysis. The Lancet Oncology 2016;17(2): 174-183.

Institute NC. Common Terminology Criteria for Adverse Events (CTCAE). U.S. Department of Health and Human Services, National Institutes of Health, National Cancer Institute, 2010.

11. Dindo D, Demartines N, Clavien P-A. Classification of surgical complications: a new proposal with evaluation in a cohort of 6336 patients and results of a survey. Annals of surgery 2004;240(2): 205213.

12. Therasse P, Arbuck SG, Eisenhauer EA, Wanders J, Kaplan RS, Rubinstein L, et al. New guidelines to evaluate the response to treatment in solid tumors. European Organization for Research and Treatment of Cancer, National Cancer Institute of the United States, National Cancer Institute of Canada. Journal of the National Cancer Institute 2000;92(3): 205-216.

13. Bisschop C, van Dijk TH, Beukema JC, Jansen RLH, Gelderblom H, de Jong KP, et al. Short-Course Radiotherapy Followed by Neoadjuvant Bevacizumab, Capecitabine, and Oxaliplatin and Subsequent Radical Treatment in Primary Stage IV Rectal Cancer: Long-Term Results of a Phase II Study. Annals of surgical oncology 2017;24(9): 2632-2638.

14. Bujko K, Wyrwicz L, Rutkowski A, Malinowska M, Pietrzak L, Krynski J, et al. Long-course oxaliplatinbased preoperative chemoradiation versus $5 \times 5$ Gy and consolidation chemotherapy for CT4 or fixed CT3 rectal cancer: results of a randomized phase III study. Annals of oncology : official journal of the European Society for Medical Oncology 2016;27(5): 834-842. 
15. Markovina S, Youssef F, Roy A, Aggarwal S, Khwaja S, DeWees T, et al. Improved Metastasis- and Disease-Free Survival With Preoperative Sequential Short-Course Radiation Therapy and FOLFOX Chemotherapy for Rectal Cancer Compared With Neoadjuvant Long-Course Chemoradiotherapy: Results of a Matched Pair Analysis. International Journal of Radiation Oncology*Biology*Physics 2017;99(2): 417-426.

16. Picardi V, Deodato F, Guido A, Giaccherini L, Macchia G, Frazzoni L, et al. Palliative Short-Course Radiation Therapy in Rectal Cancer: A Phase 2 Study. International journal of radiation oncology, biology, physics 2016;95(4): 1184-1190.

17. Tyc-Szczepaniak D, Wyrwicz L, Kepka L, Michalski W, Olszyna-Serementa M, Palucki J, et al. Palliative radiotherapy and chemotherapy instead of surgery in symptomatic rectal cancer with synchronous unresectable metastases: a phase II study. Annals of oncology : official journal of the European Society for Medical Oncology 2013;24(11): 2829-2834.

18. Wong R, Cunningham D, Barbachano Y, Saffery C, Valle J, Hickish T, et al. A multicentre study of capecitabine, oxaliplatin plus bevacizumab as perioperative treatment of patients with poor-risk colorectal liver-only metastases not selected for upfront resection. Annals of oncology : official journal of the European Society for Medical Oncology 2011;22(9): 2042-2048.

19. Folprecht G, Gruenberger T, Bechstein WO, Raab HR, Lordick F, Hartmann JT, et al. Tumour response and secondary resectability of colorectal liver metastases following neoadjuvant chemotherapy with cetuximab: the CELIM randomised phase 2 trial. The Lancet Oncology 2010;11(1): 38-47.

20. Huiskens J, van Gulik TM, van Lienden KP, Engelbrecht MR, Meijer GA, van Grieken NC, et al. Treatment strategies in colorectal cancer patients with initially unresectable liver-only metastases, a study protocol of the randomised phase 3 CAIRO5 study of the Dutch Colorectal Cancer Group (DCCG). BMC cancer 2015;15: 365.

21. Verhoef C, van der Pool AEM, Nuyttens JJ, Planting AST, Eggermont AMM, de Wilt JHW. The "LiverFirst Approach" for Patients with Locally Advanced Rectal Cancer and Synchronous Liver Metastases. Dis Colon Rectum, 2009;52(1): 23-30.

22. Ayez N, Burger JWA, van der Pool AE, Eggermont AMM, Grunhagen DJ, de Wilt JHW, et al. Longterm Results of the "Liver First" Approach in Patients With Locally Advanced Rectal Cancer and Synchronous Liver Metastases. Dis Colon Rectum, 2013;56(3): 281-287.

23. Nierop PMH, Verseveld M, Galjart B, Rothbarth J, Nuyttens J, van Meerten E, et al. The liver-first approach for locally advanced rectal cancer and synchronous liver metastases. European journal of surgical oncology : the journal of the European Society of Surgical Oncology and the British Association of Surgical Oncology 2019;45(4): 591-596. 



\section{Chapter 3}

\section{High versus low dose Stereotactic Body Radiation Therapy for hepatic metastases}

Esther N.D. Kok Edwin P.M. Jansen

Birthe C. Heeres Niels F.M. Kok

Tomas Janssen Erik van Werkhoven Fay R.K. Sanders Theo J.M. Ruers

Marlies E. Nowee Koert F.D. Kuhlmann

Clinical and Translational Radiation Oncology (2019) 


\section{Abstract}

Introduction: Stereotactic Body Radiation Therapy (SBRT) is a treatment option for patients with liver metastases. This study evaluated the impact of high versus low dose image-guided SBRT of hepatic metastases.

Methods: This is a single-center retrospective study of patients with liver metastases treated with SBRT. For analyses, patients were divided into two groups: $\leq 100 \mathrm{~Gy}$ and $>100$ Gy near-minimum Biological Effective Doses $\left(\right.$ BED $_{98 \%}$ ). The main outcomes were local control, toxicity and overall survival. Cox regression analyses were performed to determine prognostic variables on local control and overall survival.

Results: Ninety patients with 97 liver metastases (77\% colorectal) were included. Median follow-up was 28.6 months. The two-year local control rates in the $\leq 100 \mathrm{~Gy}$ and $>100$ Gy BED B8\% group were $60 \%$ (95 \% Cl: 41-80\%) and 90\% (95\% Cl: $80-100 \%$ ), respectively $(p=0.004)$. Grade 3 toxicity occurred in $7 \%$ vs $2 \%$ in the $\leq 100$ Gy and $>100$ Gy group $(p=0.23)$. Two-year overall survival rates in the $\leq 100 \mathrm{~Gy}$ and $>100$ Gy group were 48\% (95\% Cl: $32-65 \%)$ and 85\% (95\% Cl: $73-97 \%)$, respectively ( $p=$ 0.007). In multivariable Cox regression analyses, group dose and tumor volume were significantly correlated with local control (HR: 3.61; $p=0.017$ and HR: $1.01 ; p=0.005$ ) and overall survival (HR: 2.38; $p=0.005$ and HR: 1.01; $p=<0.0001$ ).

Conclusion: High dose SBRT provides significantly better local control and overall survival than low dose SBRT without increasing toxicity. When surgical resection is not feasible, high dose SBRT provides an effective and safe treatment for liver metastases. 


\section{Background}

Liver metastases are the most common malignant tumors of the liver, with main primary sites in the breast, lung, colon, and rectum [1]. In patients with colorectal cancer, 25$50 \%$ will develop either synchronous or metachronous liver metastases [2]. Surgical resection is the standard of care for limited metastatic disease and the five-year survival rates after curative surgical resection are approximately 50-60\% [3]. Unfortunately, many patients with liver metastases are not eligible for surgery due to e.g. extensive disease. In a proportion of patients with limited metastatic disease surgery is an unfavorable option as a result of age, poor performance status, co-morbidity or involvement of adjacent anatomical structures (like large blood vessels). In these patients, studies show that alternative local therapies including thermal ablation can offer good rates of local control [4-6]. Thermal ablation may, however, fail when lesions are close to the biliary structures or large blood vessels because of damage to the bile duct and heatsink respectively. Ablation is also limited in superficial lesions close to the liver capsule due to high incidence of complications [7]. Moreover, local control with this technique is significantly better when metastases are $<3 \mathrm{~cm}$ in diameter $[8,9]$.

In the past, the role of radiotherapy in the treatment of liver metastases was limited due to toxicity in surrounding healthy tissue with increased risk of radiation-induced liver disease [10]. In the last decade, stereotactic body radiation therapy (SBRT) has been developed. Phase I and II studies showed that SBRT can deliver high doses of radiation with great precision and low damage to the surrounding healthy liver tissue [11-14]. Compared to other local ablative treatments, SBRT is non-invasive and requires only brief outpatient clinic visits without hospital admission during treatment. Recently, a randomized controlled phase 2 trial compared palliative standard of care treatment with standard of care plus SBRT in patients with oligometastatic cancers [15]. They concluded that treatment with SBRT was associated with improved overall survival but showed more treatment-related deaths compared to the control group. Despite the increased amount of studies, the ultimate dose and fractionation schedule remain under debate.

At the Netherlands Cancer Institute, image-guided SBRT has been used for many years. Over time, our fractionation schedule for liver metastases changed after positive results published on high dose SBRT $[16,17]$. The aim of this study was to evaluate the efficacy and safety of high versus low dose SBRT in patients with liver metastases in our institute and to determine prognostic variables on local control and overall survival. 


\section{Methods}

This was a retrospective study of patients with liver metastases who underwent SBRT at the Netherlands Cancer Institute, a tertiary referral center for patients with cancer in the Netherlands. Consecutive patients treated with SBRT between April 2009 and April 2017 were included. Hepatic metastases from any primary solid tumor were included. Patients with primary liver cancer were excluded. Before SBRT, all patients were individually discussed in a multidisciplinary meeting including radiologists, oncologists, radiation and surgical oncologists. Indications for SBRT included inoperable liver metastases due to age, anatomic location or poor performance status and inoperable recurrent disease. Extrahepatic disease was permitted when controlled with other local or systemic treatment modalities. The study was approved by the institutional review board of the Netherlands Cancer Institute (METC18.1493/N18SBR).

\section{Treatment}

Prior to SBRT, fiducial Twist markers were placed by an interventional radiologist using computer tomography (CT) or ultrasound guidance. When patients had hepatic surgery prior to SBRT, surgical clips could be used as markers. Patients were placed in a Thorax support (Macromedics BV, Waddinxveen, the Netherlands) for immobilization and a phased contrast-enhanced CT scan was made to define the gross tumor volume (GTV), which equals the clinical target volume (CTV). When available, the CT scan was registered to planning Magnetic Resonance Imaging (MRI) in radiotherapy-position. A 4D planning CT was made to assess motion and was used to generate a mid-position CT. The contrast-enhanced CT was deformed to match the mid-position geometry [18]. The anisotropic CTV to planning target volume (PTV) margins were based on the tumor motion (8 to $15 \mathrm{~mm}$ in AP and LR direction, 9 to $16 \mathrm{~mm}$ in CC direction). Patients received a prescribed dose of $37.5-60 \mathrm{~Gy}$ in 3 fractions. Doses up to $150 \%$ of the prescribed dose was allowed in parts of the PTV that did not overlap with organs at risk. Dose constraints for organs at risk were: duodenum $\left(D_{\max }<30 \mathrm{~Gy}\right)$, heart $\left(D_{\max }\right.$ $<30 \mathrm{~Gy})$, great vessels $\left(\mathrm{D}_{\max }<45 \mathrm{~Gy}\right)$, kidneys $\left(\mathrm{V}_{15}\right.$ Gy at $\left.<35 \%\right)$, esophagus $\left(\mathrm{D}_{\max }<27\right.$ $\mathrm{Gy})$, spinal cord $\left(\mathrm{D}_{\max }<18 \mathrm{~Gy}\right)$, and stomach $\left(\mathrm{D}_{\max }<30 \mathrm{~Gy}\right)$, depending on the tumor location. A Volumetric Modulated Arc Therapy (VMAT) technique planned in Pinnacle (Philips Medical Systems, Fitchburg, WI) was used. Patients were treated on an Elekta Agility or MLCi (Elektra AB, Stockholm, Sweden) accelerator with 4D - Cone Beam CT guidance. 


\section{Follow up and Endpoints}

Standard follow-up in the outpatient clinic was performed two months after completing SBRT treatment and included an abdominal CT-scan and in individual cases an MRIscan or PET scan. Thereafter, patients were scheduled for abdominal CT-scans at fourmonth intervals.

The main outcomes were infield local control, toxicity, and overall survival. Infield local control was defined as the absence of new or re-growth of existing lesions within or at the margin of the PTV. The local control was calculated from the first day of radiation treatment until the first date of infield progression. Toxicity was evaluated using the National Cancer Institute CTCAE v4.0 criteria [19]. Acute and late toxicity were defined as toxicities within or after 3 months. Overall survival was calculated from the first day of treatment until the last date of follow up or death.

\section{Evaluation}

The follow-up scans of all patients were retrospectively reviewed by four individual observers, including two dedicated radiation oncologists, one hepatobiliary surgeon, and one liver radiologist. All relevant follow up scans were compared to the threedimensional (3D) image-based radiation treatment plan to determine if recurrence had occurred in the PTV area. Infield recurrence, out-of-field liver recurrence, and extrahepatic recurrence were individually scored by all observers. In case of interobserver disagreement, a joint meeting was planned to re-evaluate until consensus was reached.

\section{Statistical analysis}

All analyses were performed using SPSS ${ }^{\circledR}$ version 24.0 (IBM Corporation; Armonk, NY, USA) and $R$ version 3.5.1. Patients and treatment characteristics were presented as number (percentage) or median (range). For analyses, patients were divided into a $\mathrm{BED}_{98 \%}$ low dose group of $\leq 100 \mathrm{~Gy}$ and a high dose group of $>100 \mathrm{~Gy}$. The median follow up time was calculated using the reverse Kaplan-Meier method [20]. Categorical variables were compared using Fisher's test and continuous variables with the MannWhitney $U$ test. Local control and overall survival curves were calculated with the Kaplan-Meier method. For local control, patients who died from other causes without infield progression were censored. Local control was analyzed per lesion. Overall survival was analyzed per patient. The difference between the survival curves were analyzed with the Log-rank test. 
Biological effective doses (BED) were calculated according to the formula:

$B E D=n \times d \times\left(\frac{d}{a / \beta}\right)$

where $n$ is the number of fractions, $d$ the prescribed dose per fraction and $\alpha / \beta$ the fractionation sensitivity for tumor tissue of $10 \mathrm{~Gy}$. Near-minimum BED (BED ${ }_{98 \%}$ ) was defined as the dose received by $98 \%$ of the PTV volume [21, 22]. Near-maximum BED $\left(\mathrm{BED}_{2 \%}\right)$ was defined as the dose received by $2 \%$ of the PTV volume. Relative nearminimum PTV dose was defined as the dose received by $98 \%$ of the PTV volume relative to the prescribed dose and was calculated using the following formula:

Relative near PTV dose $=\frac{\text { PTV D }_{98 \%}}{n \times d}$

where PTV $D_{98 \%}$ is the near-minimum dose, $n$ is the number of fractions and $d$ the dose per fraction. Regression analyses were performed using Cox proportional hazard models. A frailty model was used to allow for clustering of lesions within a patient for the local control endpoint. Multivariable models were used to evaluate whether the association of dose group with local control and overall survival remained significant after adjusting for factors that were significant at the 0.10 level in univariable analysis. The association of local recurrence with overall survival was analyzed using a cox proportional hazard model with local recurrence as a time-dependent variable. Statistical significance was accepted with a p-value of $<0.05$.

\section{Results}

\section{Patient and treatment characteristics}

Ninety patients with 97 liver metastases were included. Primary tumor origin was colorectal in $76.7 \%$ of the patients. Extrahepatic disease was present in 29 patients (32.2\%). The median follow up of all patients was 28.6 months (range: 1.2-62.4 months), and was significantly longer in the $\leq 100$ Gygroup (54.2 months) than in the $>100$ Gy group (25.0 months) ( $p<0.001$ ). The median GTV of all tumors was $14.4 \mathrm{~cm}^{3}$ (range: $1.0-194.9$ $\mathrm{cm}^{3}$ ). No significant baseline differences were found in patient characteristics between the groups. Patient and treatment characteristics are described in Table 1.

\section{Local control}

The one-year local contol rate in the $\leq 100 \mathrm{~Gy}$ group was $67.0 \%$ (CI 95\%:50.5\%-83.5\%) and in the $>100$ Gy group $94.6 \%$ (CI 95\%: 88.9\% - 100.0\%). Two-year local control rates in these groupswere60.3\%(CI95\%:40.9\%-79.7\%)and90.3\%(CI95\%:80.3\%-100.0\%),respectively. 
Table 1. Patient and treatment characteristics

\begin{tabular}{|c|c|c|c|}
\hline Characteristics & $\leq 100$ Gy group & $>100$ Gy group & P-value \\
\hline No. of patients/treated lesions & $40 / 41$ & $50 / 56$ & \\
\hline $\begin{array}{l}\text { Sex } \\
\text { Male } \\
\text { Female }\end{array}$ & $\begin{array}{l}23(57.5) \\
17(42.5)\end{array}$ & $\begin{array}{l}29(58.0) \\
21(42.0)\end{array}$ & 1.00 \\
\hline Age at treatment (years) & $64.3(40.6-84.9)$ & $64.2(38.5-85.8)$ & 0.54 \\
\hline $\begin{array}{l}\text { Primary tumor location } \\
\text { Colorectal } \\
\text { Other gastrointestinal tumor } \\
\text { Lung } \\
\text { Breast } \\
\text { Melanoma } \\
\text { Other }\end{array}$ & $\begin{array}{c}30(75.0) \\
3(7.5) \\
3(7.5) \\
1(2.5) \\
0(0) \\
3(7.5)\end{array}$ & $\begin{array}{l}39(78.0) \\
2(4.0) \\
1(2.0) \\
3(6.0) \\
1(2.0) \\
4(8.0)\end{array}$ & 0.81 \\
\hline $\begin{array}{l}\text { Extrahepatic disease } \\
\text { None } \\
\text { Lung } \\
\text { Peritoneum } \\
\text { Lymph nodes } \\
\text { Other }\end{array}$ & $\begin{array}{l}26(65.0) \\
7(17.5) \\
3(7.5) \\
2(5.0) \\
2(5.0)\end{array}$ & $\begin{array}{c}35(70.0) \\
9(18.0) \\
1(2.0) \\
1(2.0) \\
4(8.0)\end{array}$ & 0.50 \\
\hline $\begin{array}{l}\text { Previous treatment } \\
\text { None } \\
\text { Local therapy (surgery, radiotherapy) } \\
\text { Systemic therapy } \\
\text { Combination local and systemic treatment }\end{array}$ & $\begin{aligned} 5 & (12.5) \\
1 & (2.5) \\
31 & (77.5) \\
3 & (7.5)\end{aligned}$ & $\begin{array}{c}5(10.0) \\
3(6.0) \\
31(62.0) \\
11(22.0)\end{array}$ & 0.71 \\
\hline $\begin{array}{l}\text { Volumes } \\
\qquad \begin{array}{l}\text { GTV }\left(\mathrm{cm}^{3}\right) \\
\text { PTV }\left(\mathrm{cm}^{3}\right)\end{array}\end{array}$ & $\begin{array}{c}18.4(1.3-195.0) \\
86.5(19.0-481.6)\end{array}$ & $\begin{array}{c}14.5(1.0-157.3) \\
66.0(18.4-400.4)\end{array}$ & $\begin{array}{c}0.31 \\
0.094\end{array}$ \\
\hline $\begin{array}{l}\text { Prescribed dose } \\
\text { Dose per fraction (Gy) } \\
\text { Total dose (Gy) }\end{array}$ & $\begin{array}{c}12.5(12.0-15.0) \\
37.50(36.0-45.0)\end{array}$ & $\begin{array}{l}20.0(12.0-20.0) \\
60.0(51.0-60.0)\end{array}$ & $\begin{array}{l}<0.001 \\
<0.001\end{array}$ \\
\hline $\begin{array}{l}\text { Biologically Effective Dose } \\
\qquad \mathrm{BED}_{98 \%}(\mathrm{~Gy}) \\
\mathrm{BED}_{2 \%}(\mathrm{~Gy})\end{array}$ & $\begin{array}{c}77.6(8.1-93.1) \\
143.3(71.1-336.8)\end{array}$ & $\begin{array}{l}175.2(103.7-355.8) \\
300.8(143.6-347.5)\end{array}$ & $\begin{array}{l}<0.001 \\
<0.001\end{array}$ \\
\hline Relative near-minimum PTV dose (\%) & $90.9(16.0-98.84)$ & $94.6(40.0-98.8)$ & 0.22 \\
\hline
\end{tabular}

Data are reported as number (percent) or median (range)

Abbreviation: GTV: gross tumor volume. PTV: planned target volume. $\mathrm{BED}_{98 \%}=$ near minimum $\mathrm{BED}$. $\mathrm{BED}_{2 \%}=$ near-maximum BED

A significant difference was found between the two groups $(p=0.004)$ (Figure 1). In univariable Cox regression analyses, a larger GTV was significantly associated with a worse local control (HR: 1.02; $p=0.001$ ) (Table 2). Group dose was a significant predictor of local control (HR: $4.20 ; p=0.007$ ). Multivariable Cox proportional hazard analysis was performed to evaluate whether the association of dose group with local control remained significant after adjusting for tumor volume. Since dose group (low vs high dose) is correlated to the $\mathrm{BED}_{2 \%}$ and $\mathrm{BED}_{98 \%}$, these were not included in the model. In multivariable analyses, both dose group and GTV variables were significant predictors for local control ( $p=0.017$ and $p=0.0046)$. 


\section{Chapter 3}

Figure 1. Kaplan Meier curves for local control and overall survival
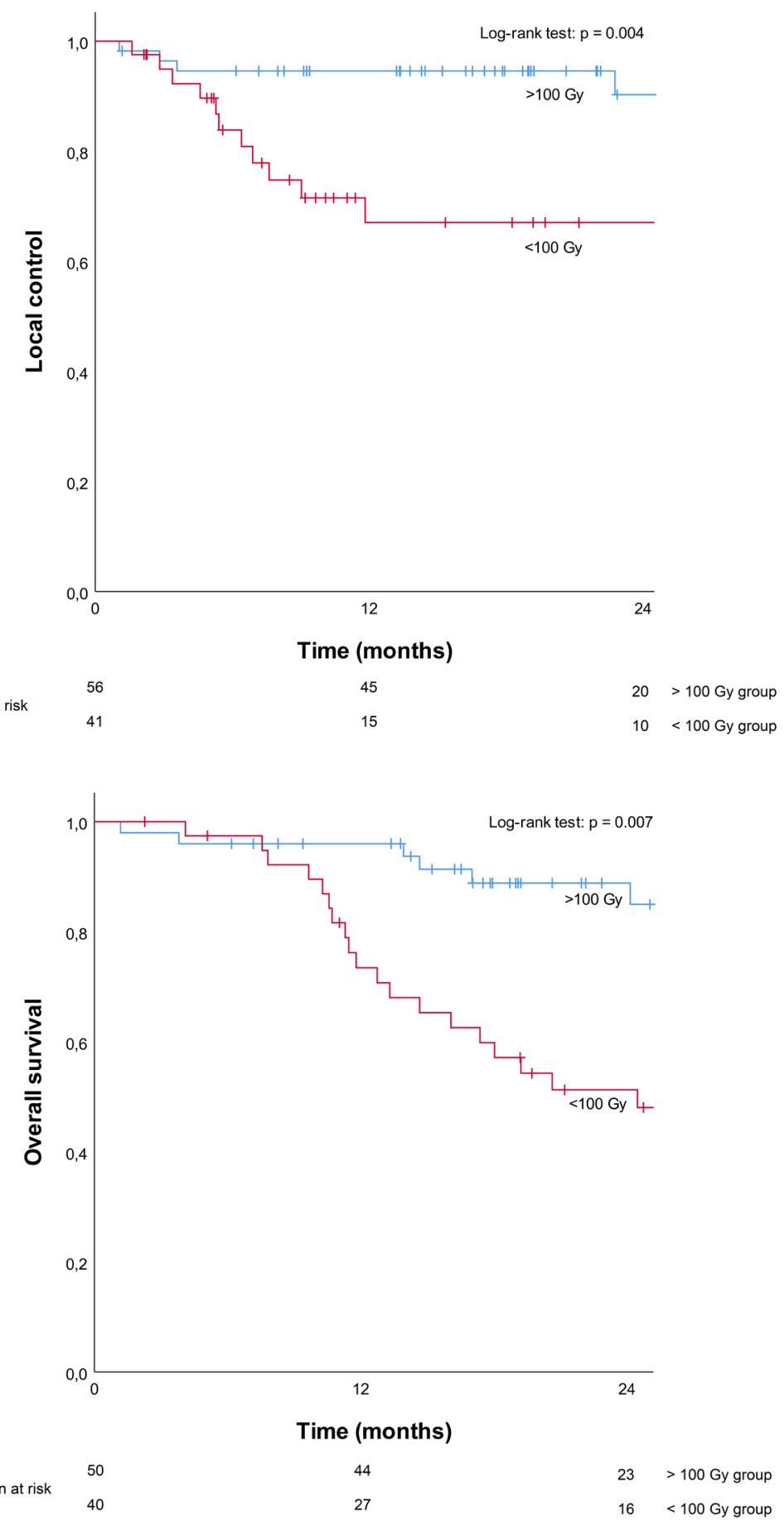
Table 2. Univariable and multivariable analyses for local control and overall survival

\begin{tabular}{|c|c|c|c|c|c|c|}
\hline \multirow[b]{2}{*}{ Variables } & \multicolumn{3}{|c|}{ Local control } & \multicolumn{3}{|c|}{ Overall survival } \\
\hline & HR & $95 \% \mathrm{Cl}$ & $P$ & HR & $95 \% \mathrm{Cl}$ & $P$ \\
\hline \multicolumn{7}{|l|}{ Univariate analysis } \\
\hline Group dose ( $\leq 100$ Gy vs. >100 Gy) & 4.20 & $1.47-11.98$ & 0.007 & 2.67 & $1.34-5.33$ & 0.005 \\
\hline Age at treatment (continues) & 1.00 & $0.95-1.05$ & 0.99 & 1.00 & $0.97-1.04$ & 0.80 \\
\hline Primary tumor (CRC vs. other) & 2.09 & $0.48-9.17$ & 0.33 & 0.76 & $0.37-1.55$ & 0.45 \\
\hline Extrahepatic disease (yes vs. no) & 0.91 & $0.32-2.57$ & 0.85 & 1.16 & $0.58-2.30$ & 0.67 \\
\hline Prior chemotherapy (yes vs. no) & 1.49 & $0.55-4.02$ & 0.43 & 1.04 & $0.54-1.99$ & 0.91 \\
\hline $\mathrm{GTV}\left(\mathrm{cm}^{3}\right)$ & 1.02 & $1.01-1.03$ & 0.001 & 1.02 & $1.01-1.02$ & $<0.001$ \\
\hline $\mathrm{BED}_{98 \%}(\mathrm{~Gy})$ per $10 \mathrm{~Gy}$ & 0.90 & $0.78-0.95$ & 0.013 & 0.90 & $0.84-0.99$ & $<0.001$ \\
\hline $\mathrm{BED}_{2 \%}(\mathrm{~Gy})$ per $10 \mathrm{~Gy}$ & 0.94 & $0.85-0.97$ & 0.029 & 0.95 & $0.91-0.99$ & 0.012 \\
\hline Relative near-min. PTV dose & 0.99 & $0.97-1.01$ & 0.20 & 0.99 & $0.90-1.01$ & 0.27 \\
\hline Infield recurrence ${ }^{1}$ & & & & 3.55 & $1.77-7.13$ & $<0.001$ \\
\hline \multicolumn{7}{|l|}{ Multivariate analysis } \\
\hline $\begin{array}{l}\text { Group dose ( } \leq 100 \text { Gy vs. }>100 \text { Gy) } \\
\text { Age at treatment (continues) } \\
\text { Primary tumor (CRC vs. other) } \\
\text { Extra hepatic disease (yes vs. no) } \\
\text { Prior chemotherapy (yes vs. no) }\end{array}$ & 3.61 & $1.25-10.40$ & 0.017 & 2.38 & $1.16-4.90$ & 0.005 \\
\hline $\begin{array}{l}\text { GTV }\left(\mathrm{cm}^{3}\right) \\
\mathrm{BED}_{98 \%}(\mathrm{~Gy}) \text { per } 10 \mathrm{~Gy} \\
\mathrm{BED}_{2 \%}(\mathrm{~Gy}) \text { per } 10 \mathrm{~Gy} \\
\text { Relative near-min. PTV dose }\end{array}$ & 1.01 & $1.00-1.02$ & 0.005 & 1.01 & $1.01-1.02$ & 0.0001 \\
\hline
\end{tabular}

'Infield recurrence as a time dependent variable

Abbreviations: HR: hazard ratio. Cl: Confidence Interval. CRC: colorectal cancer. GTV: Gross Tumor Volume. BED: Biologically Effective Dose. BED $_{98 \%}$ : near-minimum BED. BED ${ }_{2 \%}$ : near-maximum BED. PTV: Planning Target Volume

\section{Toxicity}

SBRT was well tolerated. There was no significant difference in grade $\geq 3$ treatment related-toxicity between both groups $(p=0.23)$. Acute toxicity grade 3 was observed in 2 patients of the $\leq 100$ Gy group and in 1 patient of the $>100$ Gy group, all consisting of biliary obstruction. One patient ( $\mathrm{BED}_{98 \%}: 56 \mathrm{~Gy} ; \mathrm{BED}_{2 \%}: 141 \mathrm{~Gy}$ ) developed fibrosis of the biliary ducts requiring a biliary stent. The other 2 patients $\left(\mathrm{BED}_{98 \%}: 32 \mathrm{~Gy}\right.$ and $107 \mathrm{~Gy}$; $\mathrm{BED}_{2 \%}: 245 \mathrm{~Gy}$ and $275 \mathrm{~Gy}$ ) suffered from local edema near the biliary ducts and received pharmacological treatment with dexamethasone. Late toxicity grade 3 was observed in 1 patient ( $\mathrm{BED}_{98 \%}: 79$ Gy and $\mathrm{BED}_{2 \%}: 123 \mathrm{~Gy}$ ) in the $\leq 100 \mathrm{~Gy}$ group who developed a cholecystitis caused by biliary fibrosis after which a cholecystectomy was performed. In both groups, grade 4 or 5 treatment-related toxicity was not observed.

\section{Overall surival}

Median overall survival was 32.3 months for all patients. The one and two-year overall survival rates in the $\leq 100$ Gy group were $70.8 \%$ and $48.1 \%$ (Cl: $56.2-85.3 \% ; 31.6-64.6 \%$ ), respectively. In the $>100$ Gy group the one and two-year overall survival rates were 
96.0\% and $85.0 \%$ (Cl: 90.5-100\%; 73.4-96.6\%). A significant difference was observed between the groups ( $p=0.007$ ) (Figure 1).

Univariable analyses showed that group dose, $\mathrm{BED}_{2 \%}$ and $\mathrm{BED}_{98 \%}$ had a significant impact on overall survival (Table 2$)(p=0.005, p<0.001$ and $p=0.012$ ). A larger tumor volume (GTV) was associated with a worse overall survival ( $p<0.0001)$. Infield recurrence significantly increased the chance of death (HR: 3.55; 95\% Cl: $1.77-7.13 ; p$ $<0.001)$. In multivariable analyses, dose group and GTV were significant predictors for overall survival $(p=0.019$ and $p<0.0001)$.

\section{Discussion}

This study shows that high dose image-guided SBRT provides significantly better local control when treating liver metastases than low dose SBRT. Overall survival was also significantly better in the $>100 \mathrm{~Gy}$ dose group compared to the $\leq 100 \mathrm{~Gy}$ dose group. Treatment was furthermore well tolerated with similar toxicity rates in both groups.

Previous studies investigated SBRT in the treatment of liver metastases and showed promising results $[17,23,24]$. A small phase I study of Rule et al. was one of the first that investigated dose escalation for liver metastases [14]. The authors included 27 patients who were divided into 3 cohorts who were treated with $30 \mathrm{~Gy}$ in 3 fractions, $50 \mathrm{~Gy}$ in 5 fractions and 60 Gy in 5 fractions. The two-year local control rates were $56 \%$, 89\%, and $100 \%$ respectively. Grade 3-5 toxicity was not observed. A significant difference in local control was only seen comparing the $30 \mathrm{~Gy}$ and $60 \mathrm{~Gy}$ group. More recently, a systematic review of liver SBRT has been published in which local control rates and the effect of dose regimen on local control was evaluated [25]. Thirteen articles - including 290 patients with liver metastases - were included. They showed that local control rates were significantly better for metastatic lesions treated with BED's >100 Gy compared to lesions treaded with BED's <100 Gy. In the current study, local control rates were concordant, and the difference in local control between the >100 Gy group and the $\leq 100$ Gy group was statistically significant. Consistent with these previous studies, toxicity rates remained similar regardless of the dose escalation.

In the current study, local control is correlated with overall survival. Conflicting results on the relation between local control and overall survival have been published. In some studies, local control of the liver metastases did not affect overall survival [23, 26, 27]. While other studies reported improved overall survival after adequate local control. The study of McPartlin et al. reported the long-term results of 60 patients treated with SBRT in previous phase I and II studies [28]. This study showed that infield progression after SBRT was significantly associated with overall survival (HR 2.46; $p<0.001)$. Similar results were published by Joo et al. who reported that local recurrence after SBRT is 
associated with death with a HR of $4.95(p<0.01)$ [29].

In the Netherlands Cancer Institute, liver metastases are treated with SBRT when other treatment options are not feasible. Patients can be medically not fit enough for surgery due to age or bad performance state or have inoperable metastases due to anatomic location. Thermal ablation is another common alternative to treat lesions in these patients but this technique is also limited in frail patients since it is an invasive treatment, requiring hospital admission and complete anesthesia. Despite the population treated with SBRT is expected to have worse baseline characteristics, the median overall survival of all patients was 32.3 months. This is remarkable as patients selected for SBRT were often old, frail or had recurrent disease. This study shows that SBRT is a good modality to treat liver metastases in a selected population, with the benefit of treating patients in an outpatient setting.

Whether thermal ablation or SBRT is superior for local treatment of liver metastases remains unclear. The ultimate way to compare these two treatments would be with prospective randomized trials. Unfortunately, some randomized controlled trials tried and were closed prematurely due to insufficient recruitment (NCT01233544). It is unlikely that they will occur in the near future. A recent study retrospectively compared the treatment of liver metastases with SBRT $(n=170)$ and radiofrequency ablation $($ RFA $)(n=112)[30,31]$. Both treatments provided excellent and similar local control rates for lesions $<20 \mathrm{~mm}$. For lesions $>20 \mathrm{~mm}$, SBRT was correlated with better local control. Therefore it was concluded that treatment with SBRT might be favorable over RFA in lesions larger than $20 \mathrm{~mm}$. A drawback of this study, besides the retrospective nature, was that $<25 \%$ of the patients treated with SBRT had lesions larger than $40 \mathrm{~mm}$. Therefore, SBRT local control rates of lesions larger than $40 \mathrm{~mm}$ remain unknown.

The present study had several limitations. These include selection bias, inclusion of multiple tumor types and development of treatments over time. Selection bias is inherently present in a retrospective study. The authors chose to include consecutive patients with metastases from various tumors. Tumor biology will influence local control and survival, and tumor biology certainly correlates to tumor origin [32]. Comparing two sequential historical cohorts does not correct for all other changes over time in oncology including indications for local treatment (i.e. surgical, thermal or radiation) and indication for systemic treatment and systemic treatment options. Over the past decade, some biologicals have become available for several tumors. Furthermore, imaging and radiotherapy technology has improved in the last decade but it remains unknown what the impact of these changes on local control and survival are.

This study shows that high dose SBRT provides significantly better local control rates in patients with liver metastases compared to low dose SBRT. Survival is associated 
with tumor volume and dose escalation. Toxicity rates are low and did not increase after dose escalation. When surgical resection is not feasible, high dose SBRT in patients with liver metastases is safe and effective. 


\section{References}

1. Doherty, G.M., CURRENT Diagnosis \& Treatment: Surgery. 14th ed. 2006.

2. van der Pool, A.E., R.A. Damhuis, J.N. ljzermans, et al., Trends in incidence, treatment and survival of patients with stage IV colorectal cancer: a population-based series. Colorectal Dis, 2012. 14(1): p. 56-61.

3. Zaydfudim, V.M., T.L. McMurry, A.M. Harrigan, et al., Improving treatment and survival: a populationbased study of current outcomes after a hepatic resection in patients with metastatic colorectal cancer. HPB : the official journal of the International Hepato Pancreato Biliary Association, 2015. 17(11): p. 1019-1024.

4. Gillams, A.R. and W.R. Lees, Five-year survival in 309 patients with colorectal liver metastases treated with radiofrequency ablation. Eur Radiol, 2009. 19(5): p. 1206-13.

5. Groeschl, R.T., C.H. Pilgrim, E.M. Hanna, et al., Microwave ablation for hepatic malignancies: a multiinstitutional analysis. Ann Surg, 2014. 259(6): p. 1195-200.

6. Beermann, M., J. Lindeberg, J. Engstrand, et al., 1000 consecutive ablation sessions in the era of computer assisted image guidance - Lessons learned. European journal of radiology open, 2018. 6: p. 1-8.

7. Rhim, H., S.N. Goldberg, G.D. Dodd, 3rd, et al., Essential techniques for successful radio-frequency thermal ablation of malignant hepatic tumors. Radiographics, 2001. 21 Spec No: p. S17-35; discussion S36-9.

8. Shady, W., E.N. Petre, M. Gonen, et al., Percutaneous Radiofrequency Ablation of Colorectal Cancer Liver Metastases: Factors Affecting Outcomes--A 10-year Experience at a Single Center. Radiology, 2016. 278(2): p. 601-11.

9. Gillams, A., N. Goldberg, M. Ahmed, et al., Thermal ablation of colorectal liver metastases: a position paper by an international panel of ablation experts. The interventional oncology sans frontières meeting 2013. 2015. 25(12): p. 3438-3454.

10. Lawrence, T.S., J.M. Robertson, M.S. Anscher, et al., Hepatic toxicity resulting from cancer treatment. Int J Radiat Oncol Biol Phys, 1995. 31(5): p. 1237-48.

11. Katz, A.W., M. Carey-Sampson, A.G. Muhs, et al., Hypofractionated stereotactic body radiation therapy (SBRT) for limited hepatic metastases. Int J Radiat Oncol Biol Phys, 2007. 67(3): p. 793-8.

12. Rusthoven, K.E., B.D. Kavanagh, H. Cardenes, et al., Multi-institutional phase II trial of stereotactic body radiation therapy for liver metastases. J Clin Oncol, 2009. 27(10): p. 1572-8.

13. Lee, M.T., J.J. Kim, R. Dinniwell, et al., Phase I study of individualized stereotactic body radiotherapy of liver metastases. J Clin Oncol, 2009. 27(10): p. 1585-91.

14. Rule, W., R. Timmerman, L. Tong, et al., Phase I dose-escalation study of stereotactic body radiotherapy in patients with hepatic metastases. Ann Surg Oncol, 2011. 18(4): p. 1081-7.

15. Palma, D.A., R. Olson, S. Harrow, et al., Stereotactic ablative radiotherapy versus standard of care palliative treatment in patients with oligometastatic cancers (SABR-COMET): a randomised, phase 2, open-label trial. Lancet, 2019. 393(10185): p. 2051-2058.

16. Scorsetti, M., S. Arcangeli, A. Tozzi, et al., Is stereotactic body radiation therapy an attractive option for unresectable liver metastases? A preliminary report from a phase 2 trial. Int J Radiat Oncol Biol Phys, 2013. 86(2): p. 336-42.

17. Dewas, S., J.E. Bibault, X. Mirabel, et al., Prognostic factors affecting local control of hepatic tumors treated by Stereotactic Body Radiation Therapy. Radiat Oncol, 2012. 7: p. 166.

18. Kruis, M.F., J.B. van de Kamer, J.J. Sonke, et al., Registration accuracy and image quality of time averaged mid-position CT scans for liver SBRT. Radiother Oncol, 2013. 109(3): p. 404-8.

19. Common terminology criteria for adverse events (CTCAE) v.4.0. [cited 2018 July 2018]; Available from: https://evs.nci.nih.gov/ftp1/CTCAE/CTCAE_4.03/CTCAE_4.03_2010-06-14_QuickReference_5x7. pdf.

20. Schemper, M. and T.L. Smith, A note on quantifying follow-up in studies of failure time. Control Clin Trials, 1996. 17(4): p. 343-6. 
21. Wilke, L., N. Andratschke, O. Blanck, et al., ICRU report 91 on prescribing, recording, and reporting of stereotactic treatments with small photon beams : Statement from the DEGRO/DGMP working group stereotactic radiotherapy and radiosurgery. Strahlenther Onkol, 2019. 195(3): p. 193-198.

22. Report 91. Journal of the International Commission on Radiation Units and Measurements, 2017. 14(2): p. 1-160.

23. Andratschke, N., H. Alheid, M. Allgäuer, et al., The SBRT database initiative of the German Society for Radiation Oncology (DEGRO): patterns of care and outcome analysis of stereotactic body radiotherapy (SBRT) for liver oligometastases in 474 patients with 623 metastases. BMC Cancer, 2018. 18.

24. Scorsetti, M., T. Comito, A. Tozzi, et al., Final results of a phase II trial for stereotactic body radiation therapy for patients with inoperable liver metastases from colorectal cancer. J Cancer Res Clin Oncol, 2015. 141(3): p. 543-53.

25. Ohri, N., W.A. Tome, A. Mendez Romero, et al., Local Control After Stereotactic Body Radiation Therapy for Liver Tumors. Int J Radiat Oncol Biol Phys, 2018.

26. Klement, R.J., N. Abbasi-Senger, S. Adebahr, et al., The impact of local control on overall survival after stereotactic body radiotherapy for liver and lung metastases from colorectal cancer: a combined analysis of 388 patients with 500 metastases. Radiother and Oncol 2019. 19(1): p. 173.

27. Fode, M.M. and M. Hoyer, Survival and prognostic factors in 321 patients treated with stereotactic body radiotherapy for oligo-metastases. Radiother Oncol, 2015. 114(2): p. 155-60.

28. McPartlin, A., A. Swaminath, R. Wang, et al., Long-Term Outcomes of Phase 1 and 2 Studies of SBRT for Hepatic Colorectal Metastases. Int J Radiat Oncol Biol Phys, 2017. 99(2): p. 388-395.

29. Joo, J.H., J.H. Park, J.C. Kim, et al., Local Control Outcomes Using Stereotactic Body Radiation Therapy for Liver Metastases From Colorectal Cancer. Int J Radiat Oncol Biol Phys, 2017. 99(4): p. 876-883.

30. Jackson, W.C., Y. Tao, M. Mendiratta-Lala, et al., Comparison of Stereotactic Body Radiation Therapy and Radiofrequency Ablation in the Treatment of Intrahepatic Metastases. International Journal of Radiation Oncology*Biology*Physics, 2018. 100(4): p. 950-958.

31. Franzese, C., T. Comito, E. Clerici, et al., Liver metastases from colorectal cancer: propensity scorebased comparison of stereotactic body radiation therapy vs. microwave ablation. J Cancer Res Clin Oncol, 2018.

32. Klement, R.J., M. Guckenberger, H. Alheid, et al., Stereotactic body radiotherapy for oligo-metastatic liver disease - Influence of pre-treatment chemotherapy and histology on local tumor control. Radiother Oncol, 2017. 123(2): p. 227-233. 
High versus low dose Stereotactic Body Radiation Therapy for hepatic metastases 



\section{Chapter 4}

\section{Accelerated growth of synchronous colorectal liver metastases after primary tumor resection: effects of neoadjuvant therapy}

Koert F.D. Kuhlmann

Esther N.D. Kok

Michiel Sinasappel Oleksandra Ivashchenko Joyce Sanders Ingrid Hofland Jaap D. van Buul Niels F.M. Kok Cecil Groothuizen Theo J.M. Ruers 


\section{Abstract}

Background: Discussion remains about the timing and indication of systemic therapy in patients with colorectal cancer. Currently, patients with node-positive colon cancer receive systemic therapy after the tumor is resected. Several studies have shown that removal of the tumor may induce accelerated growth of distant (micro-) metastases. The aim of this study is to evaluate whether neoadjuvant systemic therapy can inhibit the outgrowth of liver metastases after primary tumor resection.

Methods: This was a randomized controlled pilot study with a control group and three intervention groups. Patients with synchronous unresectable colorectal liver metastases with an asymptomatic primary tumor were included. The control group received only primary tumor resection and the 3 intervention groups received 2 courses of neoadjuvant systemic therapy (respectively bevacizumab, capecitabine/ oxaliplatin or all three (CAPOX-B)) before primary tumor resection. A CT scan, PET-CT scan, and blood measurements were performed prior to the neoadjuvant treatment (in the treatment groups), 1-3 days pre-operative and 4 weeks post-operative. Primary objectives were tumor volume and FDG-uptake of the liver metastases and CEA levels.

Results: In total, 48 patients were included in this study. The control group showed a significant increase of tumor volume $(p=0.028)$ and FDG-uptake $(p=0.022)$ of the liver metastases after primary tumor resection. In the three treatment groups, tumor volumes and FDG-uptake remained stable. A significant difference in tumor volume was observed between the control group and all individual treatment groups. CEA levels decreased significantly after systemic treatment with CAPOX-B and primary tumor resection $(p=0.003)$.

Conclusion: Systemic treatment prior to primary tumor resection can inhibit the outgrowth of liver metastases in patients with synchronous unresectable colorectal liver metastases. These results suggest that neoadjuvant systemic therapy should be considered before primary tumor resection in patients with unresectable disease and potentially in patients with localized colon cancer. 


\section{Introduction}

Three decades ago, cancer biologists and clinicians observed rapid outgrowth of metastases after resection of the primary tumor [1-3]. This phenomenon was first demonstrated in mice [4]. O'Reilly et al. found that the primary tumor produced a novel anti-angiogenic factor, which they named angiostatin, that inhibited the neovascularization and growth of metastases by blocking endothelial cell proliferation. In the years following, the influence of primary tumor resection on liver metastases in human colorectal cancer patients was investigated. An elevation of both intraand peritumoral vascular density was observed in synchronous colorectal liver metastases after primary tumor resection compared to synchronous metastases in patients with the primary tumor in situ [5]. The same was seen with metachronous metastases. Subsequently, the influence of these vascular effects on the growth of the liver metastases was investigated [6]. In accordance with the previous findings, results demonstrated that resection of the primary tumor increased the percentages of proliferating cells and decreased the percentage of apoptotic cells in the metastatic lesions. These preliminary data strongly suggest an inhibitory effect of the primary tumor on the outgrowth of its liver metastases. Resection of the primary tumor seems to result in abrogation of this inhibitory effect and subsequent enhanced tumor growth of the liver metastases. It may be evident that these preliminary data have direct implications for the timing of systemic therapy in colorectal cancer, as metachronous distant metastases develop in $20 \%$ of the patients during follow up [7,8].

In primary lymph node-positive colorectal cancer, adjuvant chemotherapy is generally indicated after resection of the primary tumor. The data presented above indicate that neoadjuvant chemotherapy may improve patient outcomes since it seems to affect accelerated tumor growth as observed after resection of the primary tumor. Recently, preliminary results of the international Foxtrot trial were presented. This trial randomized non-obstructing colon cancer patients between neoadjuvant and adjuvant systemic therapy or adjuvant systemic therapy alone and showed a trend towards improved 2-year cancer control. It significantly downstaged tumors and reduced incomplete resections $[9,10]$. Patients undergoing resection of the primary tumor while liver metastases remain in situ form an interesting group to study the influence of primary tumor resection and neoadjuvant therapy on metastatic growth patterns. The aim of the current pilot study was to investigate in detail the effect of preoperative treatment with the anti-angiogenic agent bevacizumab and/or chemotherapy on the metastatic tumor outgrowth in these patients after resection of the colorectal primary tumor. 


\section{Methods}

\section{Study design and patient inclusion}

This was a prospective, randomized controlled pilot study conducted in the Netherlands Cancer Institute. Patients with synchronous unresectable colorectal liver metastases were eligible for inclusion. Unresectable disease was defined as a too extensive hepatic involvement or widespread extrahepatic disease. Patients had to be 18 years or older with histological proven primary colorectal cancer. Exclusion criteria were any signs of bowel obstruction, perforation or bleeding from the primary tumor, prior systemic therapy and contra-indications for capecitabine, oxaliplatin or bevacizumab. After written informed consent was obtained, patients were randomized. The study was approved by the institutional review board (NL14650.091.07).

\section{Treatment and study procedures}

Patients were randomized in one of the four treatment groups. Patients in the control group (group A) received immediate resection of the primary tumor without any neoadjuvant treatment. Patients assigned to the experimental group B received 2 cycles of neoadjuvant treatment with bevacizumab: $7,5 \mathrm{mg} / \mathrm{kg}$ intravenous on day 1 and day 22. Those assigned to the experimental group $C$ received 2 cycles of neoadjuvant chemotherapy: oxaliplatin at $130 \mathrm{mg} / \mathrm{m}^{2}$ intravenous on day 1 and capecitabine, $1000 \mathrm{mg} / \mathrm{m}^{2}$ orally twice-daily on day $1-14$. Patients assigned to the experimental group $D$ received 2 cycles of neoadjuvant oxaliplatin $130 \mathrm{mg} / \mathrm{m}^{2}$ intravenous on day 1 and 22 , capecitabine $1000 \mathrm{mg} / \mathrm{m}^{2}$ oral twice-daily on day $1-14$ and bevacizumab 7,5 $\mathrm{mg} / \mathrm{kg}$ intravenous on day 1 and day 22. The drugs in all experimental groups were approved as first-line treatment of metastatic colorectal cancer [11, 12].

In all groups, an abdominal Computed Tomography (CT) scan was obtained 1-3 days before primary tumor resection (scan 1) and 4 weeks post-operative (scan 2). In the experimental groups ( $B, C$, and $D$ ) an additional $C T$ scan was obtained before starting chemotherapy and/or bevacizumab treatment (scan 0). Blood samples for serum and CEA analyses were collected. Additionally, biopsies of liver metastases were taken during primary tumor resection and 4 weeks after primary tumor resection (percutaneous; ultrasound-guided). A time schedule of the study procedures is shown in Figure 1.

\section{CT imaging and quantification}

Contrast-enhanced CT scans were used for quantitative assessment of the total liver and tumor volumes at each time point of the study. The scans were reconstructed on $1.0 \times 1.0 \times 1.0$ or $1.0 \times 1.0 \times 2.0 \mathrm{~mm}$ voxel grid. To extract and quantify the total liver 


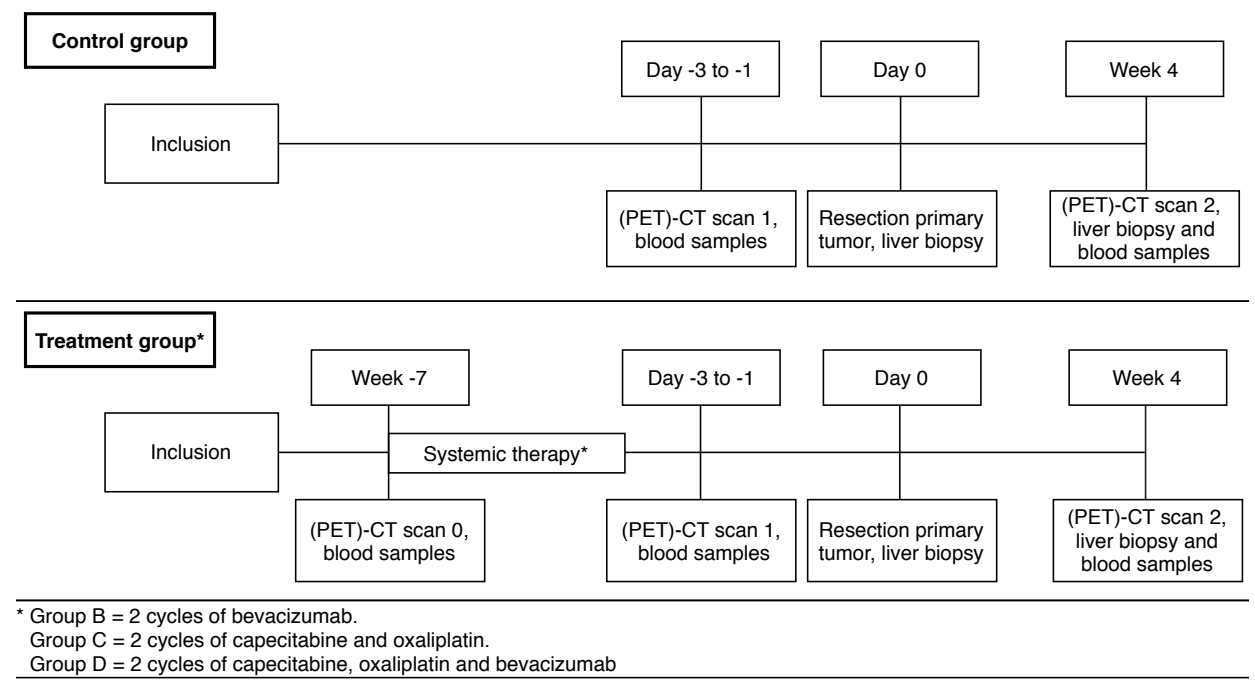

Figure 1. Time schedule of the study

and tumor volumes, each CT volume was semi-automatically segmented using the Liver Analysis module of the IntelliSpace Portal 8.0 (Philips, The Netherlands) [13]. Using the software, the liver and all tumors were carefully segmented by an experienced user with active contouring and region-growing methods.

\section{PET-CT imaging and processing of PET data}

A dedicated full-ring PET-CT-scanner (Philips Gemini ToF) was used. All patients fasted at least 6 hours prior to FDG-injection. Prior to the procedure, the patients were orally hydrated with $500 \mathrm{ml}$ of water and injected with 180-220 MBq FDG and $12 \mathrm{mg}$ furosemide (optional). One hour after intravenous injection, images of the area between the proximal femora and the base of the skull were acquired. Additionally, a low dose CT scan was performed for attenuation correction and anatomical correlation. All PET-CT acquisitions were evaluated by experienced nuclear medicine physicians. In general, liver lesions were considered tumor-positive if there is a focal increase in FDG-uptake that cannot be explained by either obstruction artifacts or a physiological process. Evaluation of the FDG-uptake was performed using the methods described previously [manuscript in preperation].

\section{Laboratory methods}

Vascular density of the liver metastases was assessed by immunohistochemical staining with anti-CD31 and anti-CD34, apoptotic cell death with caspase-3 and Ki67 was used to assess cell proliferation. Immunohistochemistry of the Formalin-Fixed Paraffin- 
Embedded (FFPE) tumor samples was performed on a BenchMark Ultra autostainer (Ventana Medical Systems (VMS)). Briefly, paraffin sections were cut at 3 um, heated at $75^{\circ} \mathrm{C}$ for 28 minutes and deparaffinized in the instrument with EZ prep solution (VMS). Heat-induced antigen retrieval was carried out using Cell Conditioning 1 (CC1, VMS) for 24 minutes at 950C (Caspase-3), 32 minutes at 950C (CD31 and CD34), or 64 minutes at 950C (Ki67). Caspase-3 was detected using clone Asp175 (1/100 dilution, 32 minutes at RT, Cell Signalling), CD31 clone JC/70A (ThermoScientific) using 1/300 dilution 32 minutes at 370C, CD34 using clone QBEnd/10 (1/4000 dilution 32 minutes at 370C, NeoMarkers / Thermo) and Ki67 using clone MIB1 (1/100 dilution, 1 hour at 370C, Agilent / DAKO). For CD34 signal amplification was applied using the Optiview Amplification Kit (4 minutes, VMS). Bound antibody was visualized using the OptiView DAB Detection Kit (VMS). Slides were counterstained with Hematoxylin and Bluing Reagent (VMS).

Caspase-3 and Ki-67 scores were determined in at least 6 randomly chosen, nonoverlapping fields (100x). Results were expressed as the percentage of nuclei that stain positive in the entire slide. Both CD31 and CD34 were scored with a semi-quantitative scoring on a scale ranging from low (1), median (2) and high (3). Furthermore, pro- and anti-angiogenetic profiles were determined by analysis of serum of patients in groups $A$ and $D$. Levels of angiostatin, endostatin, and VEGF were measured by sandwich enzyme-linked immunoassay (ELISA) according to the manufacturer's protocol. Briefly, the ELISAs are based on affinity tag-labeled capture antibody and a reporter conjugated detector antibody which immunocapture the sample analysis in the solution. To perform the assay, samples or standards were added to the wells, followed by antibody incubation and three extensive washing steps to remove unbound sample. Subsequently, TMB substrate and HRP incubation were added and yellow color is analyzed by fluorimeter at $450 \mathrm{~nm}$.

\section{Primary and secondary outcomes}

The primary objective was to evaluate differences in tumor volume of the liver metastases on CT scan and FDG uptake on PET-CT scan caused by resection of the primary tumor between the control and treatment groups. Secondary objectives were to measure any influence of the different treatment modalities on the liver metastases after primary tumor resection determined by histopathological scoring of vascular density, apoptosis, and cell proliferation, difference in tumor marker Carcinoembryonic antigen (CEA) measurements in blood and analysis of serum levels for VEGF-A, angiostatin and endostatin. Toxicity of the systemic treatment and surgical complications were analyzed. Treatment-related toxicity was registered using the 
National Cancer Institute CTCAE v4.0 [14]. The surgical complications were categorized according to the Clavien-Dindo Classification [15].

\section{Statistical analysis}

All analyses were performed using SPSS ${ }^{\circledR}$ version 24.0 (IBM Corporation; Armonk, NY, USA). Patients and treatment characteristics were presented as number (percentage) for demographic and clinical characteristics or median (range) for continuous variables. Categorical variables were compared using Fisher's test and continuous variables with the Mann-Whitney $U$ test. A Wilcoxon Signed Rank Test was used to compare differences within groups since the distribution of differences between the measurements was non-normally distributed. A Kruskal-Wallis test was used to compare differences between all groups. Tumor volumes of the liver metastases on CT scan were compared using the relative difference, expressed as a ratio between scan 1 (1 day pre-operative scan) and scan 2 (4 weeks post-operative scan) in which scan 1 was used as baseline. A similar ratio was used for CEA levels in the blood in which the pre-operative blood sample was used as the baseline. Differences on tumor volume, FDG-uptake and CEA levels are presented in boxplots. The boxplots represent the median, lower (25\%) and upper quartile (75\%) of the volumes per group. Outliers are plotted as individual points. A p-value of $\leq 0.05$ was considered statistically significant.

\section{Results}

\section{Patient inclusion and characteristics}

From August 2008 to October 2015, 48 patients with synchronous unresectable colorectal liver metastases were included. The study flowchart is shown in Figure 2. After exclusion of one patient who proved to have cholangiocarcinoma after liver biopsy, 47 patients were randomized in equal groups. Surgery in one patient in group $B$ was canceled because of progression of metastatic disease after systemic treatment and in group $C$ one patient had an unresectable primary tumor. In group $D$, one patient relocated to a different part of the Netherlands and withdrew from the study. There were no significant differences between the groups. Patient characteristics are shown in Table 1.

\section{Tumor volumes on CT scan}

Four patients were excluded from the boxplot because no contrast-enhanced CT scan was made pre-operatively (2 patients in group $B, 1$ in group $C$ and 1 in group $D$ ). Group A showed a significant increase in tumor volume of the liver metastases after primary tumor resection $(p=0.028$ ). In the treatment groups, no differences were 


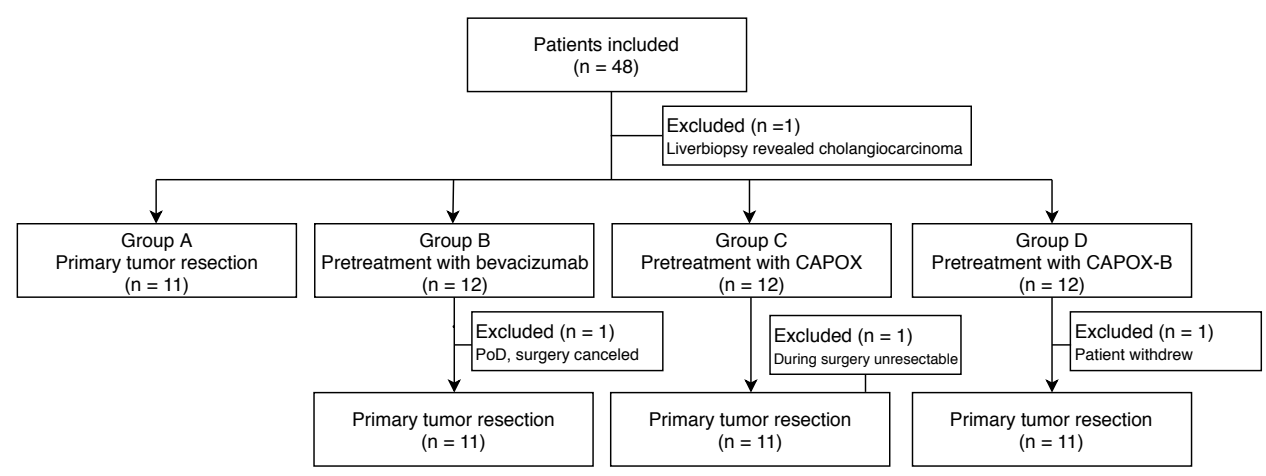

Figure 2. Flowchart of the study

Table 1. Patient characteristics

\begin{tabular}{|c|c|c|c|c|c|}
\hline Characteristics & $\begin{array}{c}\text { Group A } \\
n=11\end{array}$ & $\begin{array}{c}\text { Group B } \\
n=12\end{array}$ & $\begin{array}{c}\text { Group C } \\
n=12\end{array}$ & $\begin{array}{c}\text { Group D } \\
n=12\end{array}$ & $P$ \\
\hline $\begin{array}{l}\text { Sex } \\
\quad \text { Male } \\
\text { Female }\end{array}$ & $\begin{array}{l}4(36) \\
7(64)\end{array}$ & $\begin{array}{l}3(25) \\
9(75)\end{array}$ & $\begin{array}{l}9(75) \\
3(25)\end{array}$ & $\begin{array}{l}5(42) \\
7(58)\end{array}$ & 0.08 \\
\hline Age (years) & $56.0(40-76)$ & $60.5(33-75)$ & $65.0(52-70)$ & $63.0(48-75)$ & 0.27 \\
\hline $\begin{array}{l}\text { WHO-PS } \\
\text { WHO } 0 \\
\text { WHO } 1\end{array}$ & $\begin{array}{l}9(82) \\
2(18)\end{array}$ & $\begin{array}{c}10(83) \\
2(17)\end{array}$ & $\begin{array}{c}11(92) \\
1(8)\end{array}$ & $\begin{array}{l}8(67) \\
4(33)\end{array}$ & 0.29 \\
\hline $\begin{array}{l}\text { Primary tumor localization }{ }^{1} \\
\text { Ascending colon (incl. cecum) } \\
\text { Transverse colon } \\
\text { Descending colon } \\
\text { Recto-sigmoid }\end{array}$ & $\begin{array}{l}4(36) \\
2(18) \\
0(0) \\
6(55)\end{array}$ & $\begin{array}{l}5(42) \\
2(17) \\
1(8) \\
4(33)\end{array}$ & $\begin{array}{l}5(41) \\
1(8) \\
1(8) \\
5(41)\end{array}$ & $\begin{array}{l}6(50) \\
0(0) \\
1(8) \\
5(42)\end{array}$ & 0.83 \\
\hline No. liver metastases & $5(1-12)$ & $7(1-15)$ & $4(1-28)$ & $6(1-20)$ & 0.50 \\
\hline $\begin{array}{l}\text { Extrahepatic metastases } \\
\text { None } \\
\text { Lungs } \\
\text { Non-regional lymph nodes } \\
\text { Peritoneal } \\
\text { Bone } \\
\text { Multiple locations }\end{array}$ & $\begin{array}{c}7(64) \\
2(18) \\
1(9) \\
1(9) \\
0(0) \\
0(0)\end{array}$ & $\begin{array}{c}8(67) \\
0(0) \\
1(8) \\
1(8) \\
0(0) \\
2(17)\end{array}$ & $\begin{array}{c}9(75) \\
0(0) \\
1(8) \\
0(0) \\
1(8) \\
1(8)\end{array}$ & $\begin{array}{c}7(58) \\
1(8) \\
2(17) \\
2(17) \\
0(0) \\
0(0)\end{array}$ & 0.74 \\
\hline
\end{tabular}

Data are reported as number (percent) or median (range)

Abbreviations: WHO-PS = World health organization-performance status

'One patient in group A had 1 tumor in the ascending colon and 1 in the transverse colon

found in tumor volume between scans 1 and 2 . Figure 3 shows boxplots comparing the difference between the preoperative tumor volume and post-operative tumor volume. The median volume differences between scan 1 and 2 in the four patient groups showed a significant difference between group $A$ and $B(p=0.041)$, group $A$ and $C(p$ $=0.020)$ and group $A$ and $D(p=0.049)$. No volume differences were observed in the three treatment groups $B, C$ and $D$ between scan 0 and 1 . 


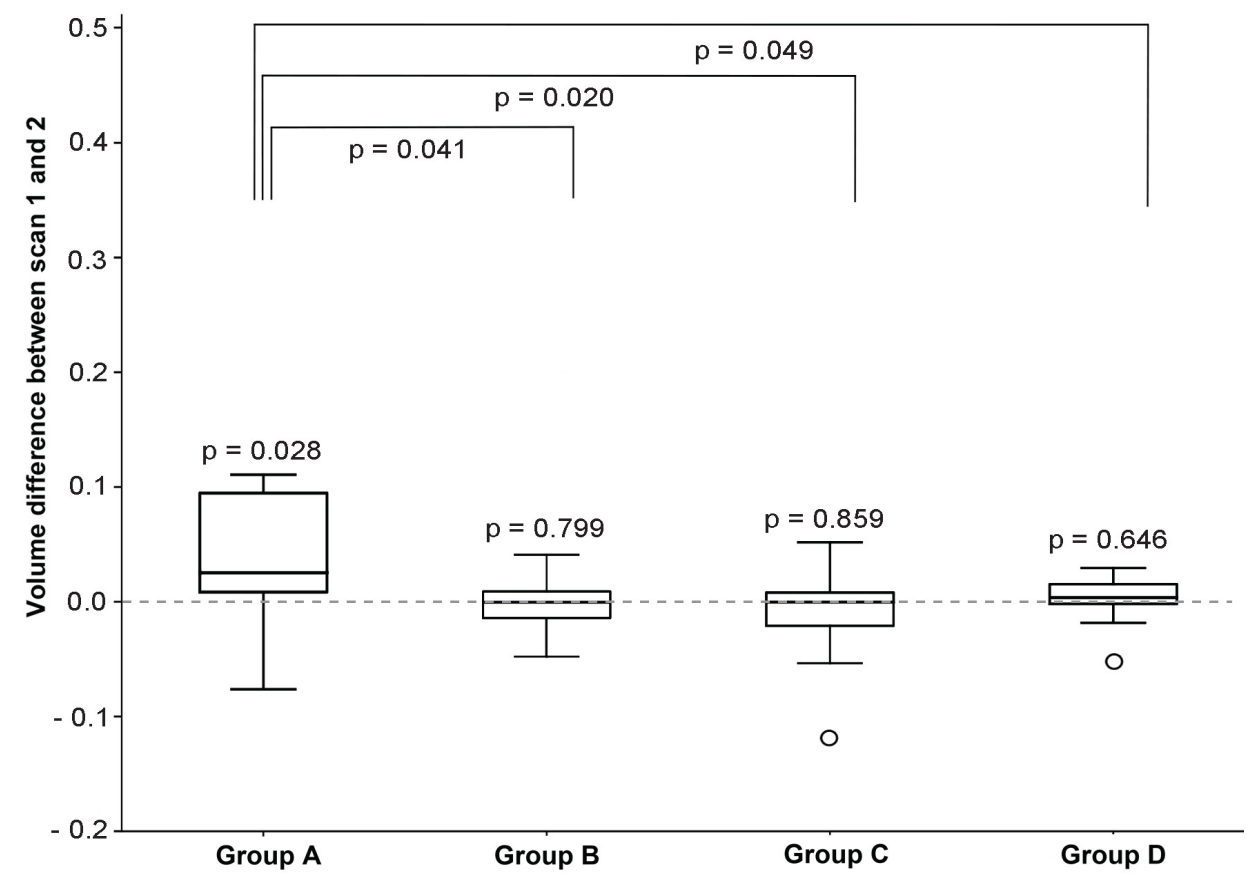

Figure 3. Boxplot of tumor volume differences between scan 1 and scan 2 in the control group (group A) and the three treatment groups (group B: bevacizumab, group C: CAPOX, group D: CAPOX-B). Scan 1 was used as the baseline.

\section{FDG-uptake on PET-CT scan}

Five patients were excluded from the boxplot because no PET-CT scan was made preoperatively ( 1 patient in group $A, 2$ patients in group $B, 1$ in group $C$ and 1 in group $D$ ). Group $A$ showed a significant increase in FDG-uptake after primary tumor resection ( $p$ $=0.022$ ) while in treatment groups $B, C$, and $D$, no significant differences were observed. Median FDG-uptake between scans 1 and 2 showed no significant difference between groups $A$ and $B(p=0.142)$, groups $A$ and $C(p=0.139)$ and group $A$ and $D(p=0.151)$. No significant differences were found comparing the other individual groups (Figure 4).

\section{CEA levels}

CEA levels of 5 patients were missing because no blood samples were taken (2 patients from group $A, 2$ from group $B$ and 1 from group $C$ ). In group $A$, an increase of CEA was seen between the pre-operative blood sample taken before the primary tumor resection (B1) and the post-operative blood sample taken 4 weeks after primary tumor resection (B2) but this was not significant $(p=0.068)$. In group $B$ and $C$, no differences were found in CEA levels between the pre- and post neoadjuvant treatment blood sample $\mathrm{B} 0$ and $\mathrm{B} 1$ or $\mathrm{B} 1$ and $\mathrm{B} 2$. In group $\mathrm{D}$, a non-significant change was observed 


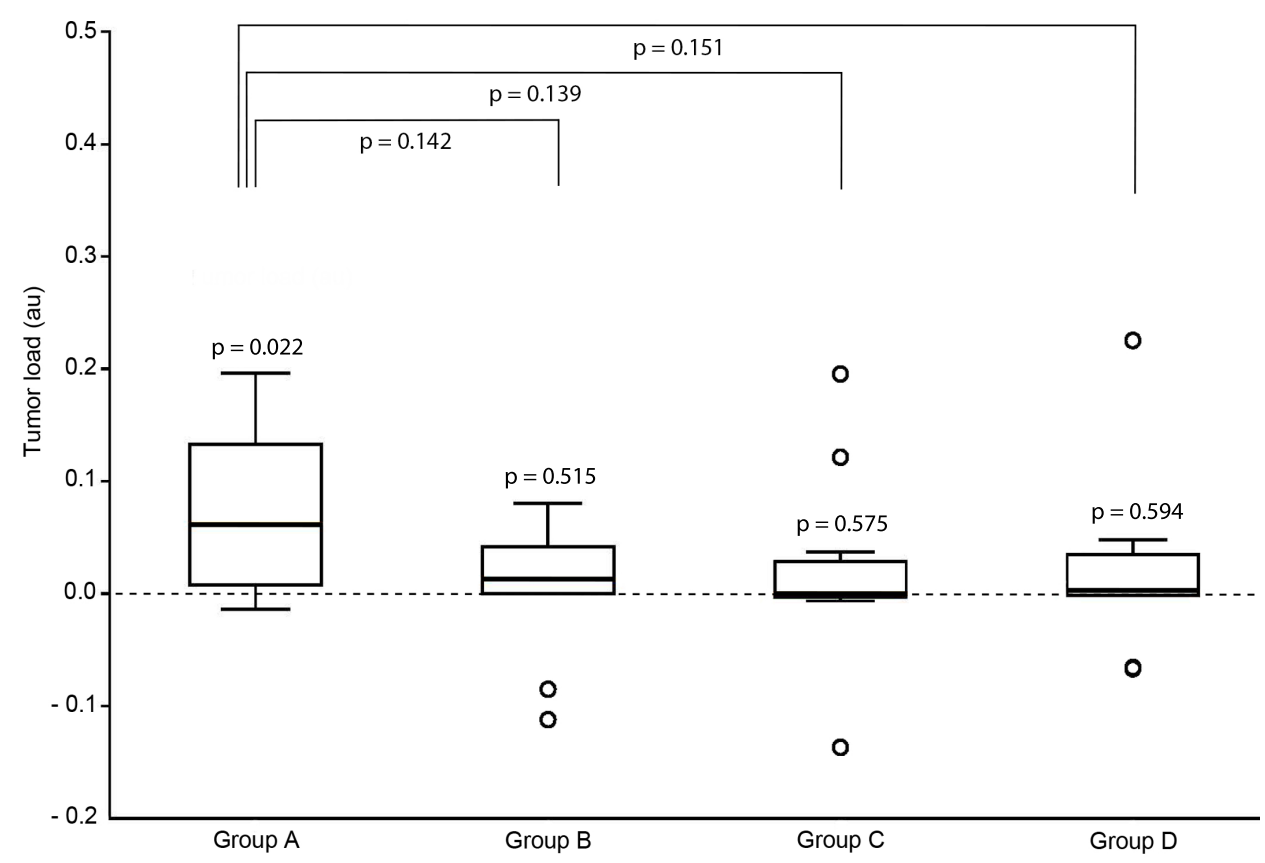

Figure 4. Boxplot of FDG-uptake differences between PET-CT scan 1 and PET-CT scan 2 in the control group (group A) and the three treatment groups (group B: bevacizumab, group C: CAPOX, group D: CAPOX-B). Scan 1 was used as the baseline.

between B0 and B1 $(p=0.075)$ and a significant decrease was found between $B 1$ and $B 2(p=0.003)$. Comparative analysis between B1 and B2 measurements in the different patient groups showed a significant difference between group $A$ and $D(p=0.003)$ but no differences between the other individual groups (Figure 5).

\section{Histopathological scoring and serum analyzes}

No differences in CD31 and CD34 staining counts, a measure for vascular density, were observed between the liver biopsies taken before and after primary tumor resection in either the control or treatment groups. The mean percentage of Ki-67 positive nuclei (cell proliferation) in group A before primary tumor resection was $73 \%$ and after primary tumor resection $77 \%(p=0.23)$ and in group $D 49 \%$ and $72 \%(p=0.67)$. In group $B$ and $C$, the mean percentage of $\mathrm{Ki} / 67$ positive nuclei before and after primary tumor resection were $70 \%$ and $60 \%(p=0.89)$ and $53 \%$ to $40 \%(p=0.59)$. No significant differences were found in the control and treatment groups in the mean percentage of Caspase-3 (apoptotic cell death) before and after primary tumor resection (Group A: $35 \%$ to $45 \%, p=0.29$; group B: $27 \%$ to $42 \%, p=0.29$; group C: $11 \%$ to $25 \%, p=0.28$ and group D: $18 \%$ to $33 \%, p=0.27$ ). 


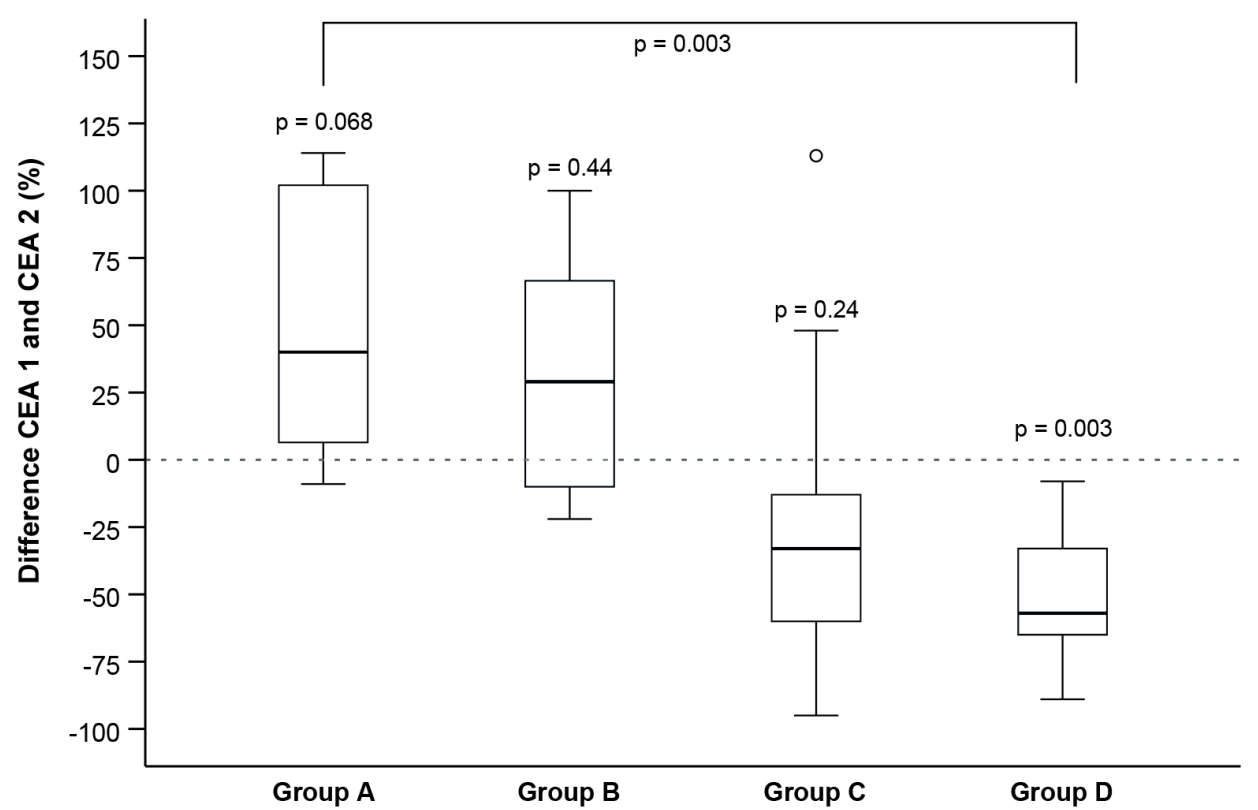

Figure 5. Boxplot of differences in preoperative and postoperative CEA levels of the control group (group A) and the three treatment groups (group B: bevacizumab, group C: CAPOX, group D: CAPOX-B). The preoperative blood sample was used as the baseline.

The median endostatin serum levels in group A before and after primary tumor resection were $1.67 \mathrm{ng} / \mathrm{ml}$ (range: $0.77-4.98 \mathrm{ng} / \mathrm{ml}$ ) and $1.86 \mathrm{ng} / \mathrm{ml}$ (range: 0.74-4.24 $\mathrm{ng} / \mathrm{ml}$ ), respectively. In group $D$, the median serum levels were $3.62 \mathrm{ng} / \mathrm{ml}$ and 3.45 $\mathrm{ng} / \mathrm{ml}$. The median angiostatin levels in group A preoperative and post-operative were $201 \mathrm{ng} / \mathrm{ml}$ and $192 \mathrm{ng} / \mathrm{ml}$. In group D, the angiostatin levels were $161 \mathrm{ng} / \mathrm{ml}$ and $197 \mathrm{ng} / \mathrm{ml}$. The median VEGF serum levels in group A before and after primary tumor resection were $10.56 \mathrm{ng} / \mathrm{ml}$ and $13.25 \mathrm{ng} / \mathrm{ml}$, respectively. In group $\mathrm{D}$, the serum levels were $15.29 \mathrm{ng} / \mathrm{ml}$ and $19.19 \mathrm{ng} / \mathrm{ml}$. No significant differences were found between the pre- and postoperative moments in the two groups.

\section{Neoadjuvant treatment tolerance and surgical characteristics}

In total, $91.7 \%$ of the patients completed the full systemic therapy. In group C, 2 patients received only 1 cycle of CAPOX because of bowel obstruction leading to early primary tumor resection. In group D, the second cycle of CAPOX-B was discontinued in 1 patient due to hypertension (grade 3). No significant differences in toxicity were found between the groups.

Midline laparotomy was performed in all procedures. In one patient in group B, surgery was canceled because of progressive liver and lung metastases after pretreatment 
with bevacizumab. One patient in group $C$ was found unresectable during surgery. No differences were seen between the groups regarding the surgical procedures or complications. Neoadjuvant treatment and surgical characteristics are shown in Table 2.

Table 2. Neoadjuvant treatment and surgical characteristics

\begin{tabular}{|c|c|c|c|c|c|}
\hline & Group A & Group B & Group C & Group D & $P$ \\
\hline Systemic treatment & & 12 & 12 & 12 & \\
\hline Completed 2 cycles & & $12(100)$ & $10(83)$ & $11(92)$ & 0.34 \\
\hline $\begin{array}{l}\text { Treatment toxicitya } \\
\qquad \begin{array}{l}\text { Grade } 1 \text { - } 2 \\
\text { Grade } 3 \\
\text { Grade } 4 \text { - } 5\end{array}\end{array}$ & & $\begin{array}{l}1(8) \\
0(0) \\
0(0)\end{array}$ & $\begin{array}{l}0(0) \\
1(8) \\
0(0)\end{array}$ & $\begin{array}{c}2(18) \\
1(9) \\
0(0)\end{array}$ & 0.41 \\
\hline $\begin{array}{l}\text { Toxicity } \\
\text { Gastrointestinal complications } \\
\text { Allergic reaction } \\
\text { Coronary spasm } \\
\text { Hypertension } \\
\end{array}$ & & $\begin{array}{l}0(0) \\
1(8) \\
0(0) \\
0(0)\end{array}$ & $\begin{array}{l}0(0) \\
0(0) \\
1(8) \\
0(0)\end{array}$ & $\begin{array}{l}2(18) \\
0(0) \\
0(0) \\
1(9) \\
\end{array}$ & \\
\hline Surgery $^{1}$ & 11 & 11 & 12 & 11 & \\
\hline $\begin{array}{l}\text { Surgical procedures } \\
\text { Right hemicolectomy } \\
\text { Left hemicolectomy } \\
\text { Low anterior resection } \\
\text { Hartmann's procedure } \\
\text { (Recto-)Sigmoid resection } \\
\text { Transversum resection } \\
\text { During surgery stopped }\end{array}$ & $\begin{array}{l}5(45) \\
0(0) \\
3(27) \\
1(9) \\
2(18) \\
0(0) \\
0(0)\end{array}$ & $\begin{array}{l}5(42) \\
1(8) \\
0(0) \\
0(0) \\
3(25) \\
2(17) \\
0(0)\end{array}$ & $\begin{array}{l}5(42) \\
1(8) \\
2(17) \\
2(17) \\
1(8) \\
0(0) \\
1(8)\end{array}$ & $\begin{array}{l}6(50) \\
0(0) \\
1(8) \\
2(17) \\
3(27) \\
0(0) \\
0(0)\end{array}$ & 0.70 \\
\hline Stoma at resection & $6(55)$ & $2(16)$ & $6(50)$ & $4(33)$ & 0.32 \\
\hline $\begin{array}{l}\text { Complications } \\
\text { None } \\
\text { Clavien Dindo I } \\
\text { Clavien Dindo II } \\
\text { Clavien Dindo III }\end{array}$ & $\begin{array}{l}8(73) \\
0(0) \\
2(18) \\
1(9)\end{array}$ & $\begin{array}{l}4(33) \\
2(17) \\
3(25) \\
2(17)\end{array}$ & $\begin{array}{l}7(58) \\
1(8) \\
2(17) \\
2(17)\end{array}$ & $\begin{array}{l}9(75) \\
1(8) \\
2(17) \\
0(0)\end{array}$ & 0.17 \\
\hline $\begin{array}{l}\text { Complications }{ }^{2} \\
\text { Wound/abdominal/thorax infection } \\
\text { Pulmonary embolism/thrombosis } \\
\text { Postoperative hypotension } \\
\text { Delirium } \\
\text { lleus } \\
\text { Anastomotic leakage } \\
\text { Platzbauch }\end{array}$ & $\begin{array}{l}1(9) \\
1(9) \\
0(0) \\
0(0) \\
1(9) \\
0(0) \\
0(0)\end{array}$ & $\begin{array}{l}4(33) \\
1(8) \\
0(0) \\
0(0) \\
2(17) \\
2(17) \\
0(0)\end{array}$ & $\begin{array}{c}2(16) \\
0(0) \\
1(8) \\
0(0) \\
1(8) \\
0(0) \\
1(8)\end{array}$ & $\begin{array}{l}1(8) \\
0(0) \\
0(0) \\
1(8) \\
1(8) \\
0(0) \\
0(0)\end{array}$ & \\
\hline Hospitalization days & $6(5-19)$ & $7(4-28)$ & $6(5-17)$ & $7(4-12)$ & 0.79 \\
\hline
\end{tabular}

'In group B, surgery was canceled due to progression of disease in 1 patient. In group D, 1 patient withdrew from the study before surgery

${ }^{2} 1$ patient in group B had anastomotic leakage, ileus, and thrombosis

\section{Discussion}

This randomized controlled pilot study demonstrates that outgrowth of metastatic disease in colorectal cancer, as observed after resection of the primary tumor, can 
be inhibited by neoadjuvant chemotherapy with or without targeted therapy before primary tumor resection. This approach was feasible with acceptable toxicity and surgical complication rates in all treatment groups. Furthermore, CEA levels of patients treated with neoadjuvant CAPOX-B decreased significantly after primary tumor resection compared to patients in the control group.

Several studies have demonstrated that primary tumor resection induces accelerated growth of distant metastases [16, 17]. In patients with colorectal liver metastases, progressive liver metastases are the most common cause of death [18]. Preventing outgrowth of metastases might improve the prognosis for these patients. The current study demonstrates that patients who received primary tumor resection without neoadjuvant treatment, show significant growth of the liver metastases and increased FDG-uptake, which is consistent with observations in earlier studies [5, 6, 19$]$. Volumes of the metastases in the treatment groups remained stable, suggesting that neoadjuvant therapy can inhibit outgrowth of (micro)metastases after primary tumor resection.

Neoadjuvant systemic therapy is not generally accepted for colorectal cancer patients. Patients with resectable colorectal cancer might show disease progression during the neoadjuvant treatment phase and emergency surgery in case of bowel obstruction, perforation or bleeding during neoadjuvant therapy is associated with adverse disease-free survival and overall survival [20]. In the current study, one patient in group B showed progression of disease with multiple new lung and lymph node metastases during treatment with bevacizumab, a treatment with a limited effect as monotherapy [21]. In one patient in group $C$, the primary tumor was found to be unresectable after neoadjuvant treatment with capecitabine - oxaliplatin during surgery, but there were no preoperative radiological signs of unresectable disease. Overall, toxicity and surgical complications of the different neoadjuvant treatment regimens were low and no emergency resections were performed. These results reflect the preliminary results of a recently performed phase III trial (FOxTROT) in 1052 patients with locally advanced colon carcinoma investigating the role of neoadjuvant therapy. $[9,10]$ Although this study could not show a significant decrease in 2-year recurrence rate $(p=0.08)$, tumors were downstaged and the positive resection margin rate was lower.

In our study, an increase of CEA was observed after primary tumor resection in the control group while a significant decrease of CEA was observed in the group who received neoadjuvant CAPOX-B. These results are in line with the tumor volume and FDG-uptake changes after resection of the primary tumor. Although CEA is widely used for the follow-up of colorectal cancer patients, CEA level changes must be 
interpreted with care to monitor chemotherapy response in the first weeks after the start of chemotherapy, because spurious early rises of CEA may occur especially after oxaliplatin [22].

The drop in metabolic activity of liver metastases observed with PET-CT in patients in the treatment groups is a well-known phenomenon. Sensitivity to detect liver metastases on PET-CT in patients after neoadjuvant therapy is significantly lower compared to patients who did not receive this treatment [23, 24]. This could be explained by the decrease in size of the metastases, but more likely due to the metabolic shutdown caused by neoadjuvant therapy.

Metastatic growth of the metastases was described to be related to the loss of angiogenesis inhibition of the primary tumor $[5,6]$. This caused an increase in vascular density and a shift in proliferation and apoptotic index in the liver metastases. These findings could not be confirmed by immunohistochemistry of the liver biopsies in the current study. Neither differences in angiostatin, endostatin or VEGF levels were seen. An explanation could be found in the small treatment groups and the tissue heterogeneity within the liver lesions $[25,26]$. Treatment response might be different in the various regions of the heterogeneous lesion, resulting in inconclusive results.

Although this pilot study shows interesting results, it is limited by the small sample size. In addition, some data were missing or could not be analyzed for various reasons. Five of the CT scans were not contrast-enhanced CT scans and could not be used for volume extraction of the tumor and in some patients immunohistochemical staining could not be performed because biopsies did not include tumor tissue or too little tumor tissue.

In summary, this randomized pilot study confirms increased metastatic growth after primary tumor surgery in patients without neoadjuvant therapy. In patients treated with neoadjuvant systemic therapy, growth of the metastases seems inhibited after primary tumor resection. Neoadjuvant treatment with capecitabine, oxaliplatin and bevacizumab appear to provide the strongest inhibition. These findings suggest that neoadjuvant systemic therapy should be considered before resection of the primary tumor. 


\section{References}

1. Gorelik E, Segal S, Feldman M. On the mechanism of tumor 'concomitant immunity'. Int J Cancer, 1981;27(6):847-856.

2. Sugarbaker E, Thornthwaite J, Ketcham AJPicr, therapy. Inhibitory effect of a primary tumor on metastasis. Prog Cancer Ther Res, 1977;5:227-240.

3. Fisher B, Gunduz N, Saffer EA. Influence of the interval between primary tumor removal and chemotherapy on kinetics and growth of metastases. Cancer research. 1983;43(4):1488-1492.

4. O'Reilly MS, Holmgren L, Shing Y, et al. Angiostatin: a novel angiogenesis inhibitor that mediates the suppression of metastases by a Lewis lung carcinoma. Cell. 1994;79(2):315-328.

5. Peeters CF, Westphal JR, de Waal RM, Ruiter DJ, Wobbes T, Ruers TJ. Vascular density in colorectal liver metastases increases after removal of the primary tumor in human cancer patients. International journal of cancer. 2004;112(4):554-559.

6. Peeters CF, de Waal RM, Wobbes T, Westphal JR, Ruers TJ. Outgrowth of human liver metastases after resection of the primary colorectal tumor: a shift in the balance between apoptosis and proliferation. International journal of cancer. 2006;119(6):1249-1253.

7. van der Pool AE, Damhuis RA, ljzermans JN, et al. Trends in incidence, treatment and survival of patients with stage IV colorectal cancer: a population-based series. Colorectal disease : the official journal of the Association of Coloproctology of Great Britain and Ireland. 2012;14(1):56-61.

8. Elferink MA, de Jong KP, Klaase JM, Siemerink EJ, de Wilt JH. Metachronous metastases from colorectal cancer: a population-based study in North-East Netherlands. International journal of colorectal disease. 2015;30(2):205-212.

9. Foxtrot Collaborative G. Feasibility of preoperative chemotherapy for locally advanced, operable colon cancer: the pilot phase of a randomised controlled trial. The Lancet Oncology. 2012;13(11):1152-1160.

10. Seymour MT, Morton D, Investigators obotIFT. FOxTROT: an international randomised controlled trial in 1052 patients (pts) evaluating neoadjuvant chemotherapy (NAC) for colon cancer. 2019;37(15_suppl):3504-3504.

11. Saltz LB, Clarke S, Diaz-Rubio E, et al. Bevacizumab in combination with oxaliplatin-based chemotherapy as first-line therapy in metastatic colorectal cancer: a randomized phase III study. Journal of clinical oncology : official journal of the American Society of Clinical Oncology. 2008;26(12):2013-2019.

12. Cassidy J, Tabernero J, Twelves C, et al. XELOX (capecitabine plus oxaliplatin): active first-line therapy for patients with metastatic colorectal cancer. Journal of clinical oncology : official journal of the American Society of Clinical Oncology. 2004;22(11):2084-2091.

13. Coulon P. BdF, Müller D., Steinberg A., Valette P.J. Clinical uses for CT Liver Analysis application. 2013; https://www.knowledgescape.philips.com/CBTSite/Media/HighlightsCTLiverAnalysis.pdf.

14. Institute NC. Common Terminology Criteria for Adverse Events (CTCAE). U.S. Department of Health and Human Services, National Institutes of Health, National Cancer Institute; 2010.

15. Dindo D, Demartines N, Clavien P-A. Classification of surgical complications: a new proposal with evaluation in a cohort of 6336 patients and results of a survey. Annals of surgery. 2004;240(2):205213.

16. van der Bij GJ, Oosterling SJ, Beelen RH, Meijer S, Coffey JC, van Egmond M. The perioperative period is an underutilized window of therapeutic opportunity in patients with colorectal cancer. Annals of surgery. 2009;249(5):727-734.

17. Demicheli R, Retsky MW, Hrushesky WJ, Baum M, Gukas ID. The effects of surgery on tumor growth: a century of investigations. Annals of oncology : official journal of the European Society for Medical Oncology. 2008;19(11):1821-1828.

18. Helling TS, Martin M. Cause of death from liver metastases in colorectal cancer. Annals of surgical oncology. 2014;21(2):501-506.

19. Scheer MG, Stollman TH, Vogel WV, Boerman OC, Oyen WJ, Ruers TJ. Increased metabolic activity of 
indolent liver metastases after resection of a primary colorectal tumor. Journal of nuclear medicine: official publication, Society of Nuclear Medicine. 2008;49(6):887-891.

20. Littlechild J, Junejo M, Simons A-M, Curran F, Subar D. Emergency resection surgery for colorectal cancer: Patterns of recurrent disease and survival. World J Gastrointest Pathophysiol. 2018;9(1):8-17.

21. Giantonio BJ, Catalano PJ, Meropol NJ, et al. Bevacizumab in combination with oxaliplatin, fluorouracil, and leucovorin (FOLFOX4) for previously treated metastatic colorectal cancer: results from the Eastern Cooperative Oncology Group Study E3200. J Clin Oncol, 2007;25(12):1539.

22. Locker GY, Hamilton S, Harris J, et al. ASCO 2006 update of recommendations for the use of tumor markers in gastrointestinal cancer. 2006;24(33):5313-5327.

23. van Kessel CS, Buckens CF, van den Bosch MA, van Leeuwen MS, van Hillegersberg R, Verkooijen HM. Preoperative imaging of colorectal liver metastases after neoadjuvant chemotherapy: a metaanalysis. Annals of surgical oncology. 2012;19(9):2805-2813.

24. Lubezky N, Metser U, Geva R, et al. The role and limitations of 18-fluoro-2-deoxy-D-glucose positron emission tomography (FDG-PET) scan and computerized tomography (CT) in restaging patients with hepatic colorectal metastases following neoadjuvant chemotherapy: comparison with operative and pathological findings. Journal of gastrointestinal surgery : official journal of the Society for Surgery of the Alimentary Tract. 2007;11(4):472-478.

25. Molinari C, Marisi G, Passardi A, Matteucci L, De Maio G, Ulivi P. Heterogeneity in Colorectal Cancer: A Challenge for Personalized Medicine? Int J Mol Sci. 2018;19(12):3733.

26. Goasguen N, de Chaisemartin C, Brouquet A, et al. Evidence of heterogeneity within colorectal liver metastases for allelic losses, mRNA level expression and in vitro response to chemotherapeutic agents. Int J Cancer, 2010;127(5):1028-1037. 
Effects of neoadjuvant therapy on primary tumor resection induced growth of colorectal liver metastases 



\section{Chapter 5}

\section{Hyperspectral imaging for tissue classification, a way toward smart laparoscopic colorectal surgery}

Esther N.D. Kok

Elisabeth J.M. Baltussen Susan G. Brouwer de Koning Joyce Sanders Arend G.J. Aalbers

Niels F.M. Kok Geerard L. Beets Claudie C. Flohil

Sjoerd C. Bruin Koert F.D. Kuhlmann Henricus J.C.M. Sterenborg Theo J.M. Ruers 


\section{Abstract}

In the last decades, laparoscopic surgery has become the gold standard in patients with colorectal cancer. To overcome the drawback of reduced tactile feedback, realtime tissue classification could be of great benefit. In this ex vivo study, hyperspectral imaging ( $\mathrm{HSI}$ ) was used to distinguish tumor tissue from healthy surrounding tissue. A sample of fat, healthy colorectal wall, and tumor tissue was collected per patient and imaged using two hyperspectral cameras, covering the wavelength range from 400 to $1700 \mathrm{~nm}$. The data were randomly divided into a training (75\%) and test (25\%) set. After feature reduction, a quadratic classifier and support vector machine were used to distinguish the three tissue types. Tissue samples of 32 patients were imaged using both hyperspectral cameras. The accuracy to distinguish the three tissue types using both hyperspectral cameras was 0.88 (STD: 0.13 ) on the test dataset. When the accuracy was determined per patient, a mean accuracy of 0.93 (STD: 0.12) was obtained on the test dataset. This study shows the potential of using $\mathrm{HSI}$ in colorectal cancer surgery for fast tissue classification, which could improve clinical outcome. Future research should be focused on imaging entire colon/rectum specimen and the translation of the technique to an intraoperative setting. 


\section{Background}

Colorectal cancer is the third most commonly diagnosed cancer worldwide and the fourth cause of death due to cancer [1, 2]. For patients with colorectal cancer, surgery is the cornerstone of the treatment. In the last decades, laparoscopic surgery for colorectal cancer has become common practice. Randomized controlled trials have proven similar clinical outcomes for laparoscopic surgery as for open surgery with a decrease in hospital stay [3]. One of the drawbacks of laparoscopic surgery is the reduced tactile feedback during surgery $[4,5]$. The lack of tactile feedback makes tissue recognition more cumbersome, especially in areas where radical resection margins are often compromised, like in locally advanced tumors and in rectal cancer. Hence, an alternative technique that would enable the surgeon to distinguish tumor from normal tissue during laparoscopic surgery in real-time would be of great benefit to secure radical resection in difficult areas, such as in rectal cancer. We will investigate the use of hyperspectral imaging (HSI) as a tool to ensure radical margins in these circumstances, distinguishing tumor from healthy colorectal tissue.

In $\mathrm{HSI}$, a broadband light source is used to illuminate an object, like, e.g., tissue. The light will interact with the tissue by reflection, scattering, and absorption of the photons. This interaction strongly depends on the tissue type and wavelength [6]. After several interactions within the tissue, part of the light will be reflected to the surface of the tissue and is detected by the hyperspectral camera. In the resulting hyperspectral image, the tissue specific spectral changes of the light can be analyzed. Ultimately, HSI result in $2 \mathrm{D}$ images of the object obtained over several wavelengths, resulting in a $3 \mathrm{D}$ datacube with two spatial dimensions and in the third dimension the wavelengths (Figure 1).
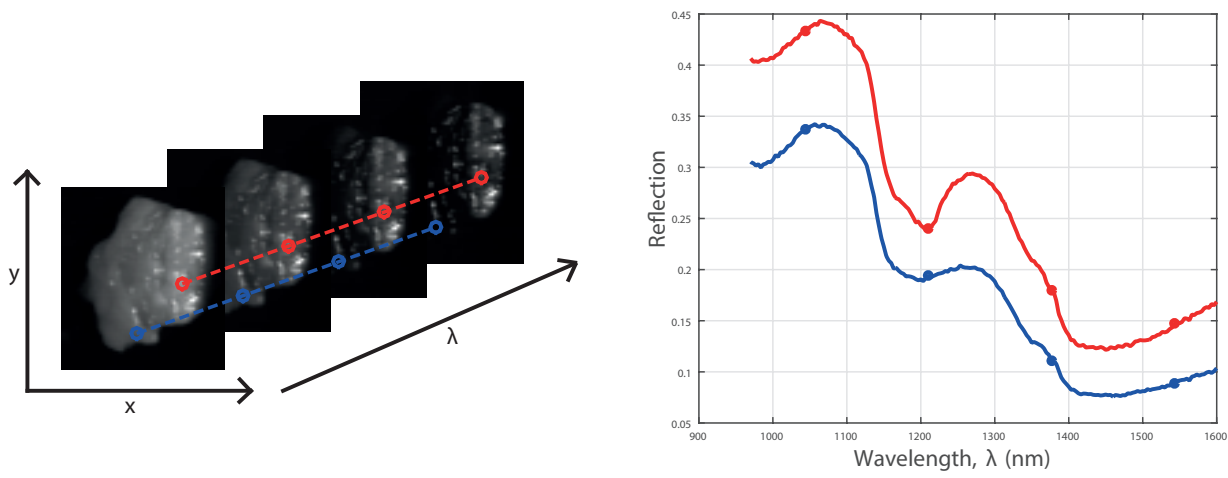

Figure 1. On the left side a hyperspectral image with two spatial dimensions $(x, y)$ and one spectral dimension $(\lambda)$. On the right side, the spectra of the two selected pixels are shown. 
Previous studies used HSI as a diagnostic tool in cancer of the cervix, breast, skin, tongue, head and neck, gastric, and colon and rectum [8-22]. In colorectal cancer, most studies focused on $\mathrm{HSI}$ of the hematoxylin-eosin (H\&E) pathology slides or tissue classification during endoscopy to distinguish tumor from healthy tissue [15-20, 21]. In the current ex vivo study, we investigated the use of HSI to differentiate normal colorectal tissue from tumor tissue in a surgical setting looking from the surface of the tissue instead of from the lumen of the colon. To this end, colorectal cancer samples obtained during surgery were imaged using $\mathrm{HSI}$ in the visible- to near-infrared region. The spectra obtained from these images were classified using a classification algorithm and were verified with histology. Finally, complete hyperspectral images were classified using the trained classifier. The ultimate goal is to develop a realtime technique for tissue identification in laparoscopic colorectal surgery.

\section{Materials and Methods}

\section{Hyperspectral Cameras}

Two hyperspectral cameras were used for the measurements, one in the visual wavelength range and one in the near-infrared wavelength range. The first was a SPECIM (Spectral Imaging Ltd., Finland) spectral camera (PFD-CL-65-V10E) with a wavelength range from 400 to $1000 \mathrm{~nm}$, a CMOS sensor of $1 \times 1312$ pixels, and a spectral resolution of $3.0 \mathrm{~nm}$, hereafter referred to as the visual camera. The second was also a SPECIM spectral camera (VLNIR CL-350-N17E) with a wavelength range from 900 to $1700 \mathrm{~nm}$, an InGaAs sensor of $1 \times 320$ pixels, and a spectral resolution of 5.0 $\mathrm{nm}$, hereafter referred to as the near-infrared camera. Both cameras were push broom cameras, meaning that they image a single line ( $x$-axis) only. To obtain a full 2-D image, the samples were placed on a translational stage and pushed underneath the camera (y-axis). All samples were illuminated using a halogen light source.

Both a dark and white reference image were taken before each measurement. The dark reference image was taken by closing the shutter of the cameras. The white reference image was taken on a Spectralon reflectance standard. The linear behavior of the visual camera allowed for a simple calibration of the camera using Equation 1. In Equation 1, $\mathrm{x}_{\text {cal }}$ is the calibrated spectrum, $\mathrm{x}$ is the original spectrum, $\mathrm{D}_{\text {ref }}$ is the dark reference, and $\mathrm{W}_{\text {ref }}$ is the white reference. The near-infrared camera, however, had a slight nonlinear behavior and was therefore calibrated using a fourth order polynomial, Equation 2, instead of the linear formula [23]. In Equation 2, $b_{i}$ are variables determined using a series of five reference samples. The values of $b_{i}$ differ per pixel and wavelength. Furthermore, $x$ is the original spectrum and $x_{\text {cal }}$ is the calibrated spectrum [24]: 
Equation 1. $\quad x_{\text {cal }}=\frac{x-D_{\text {ref }}}{W_{\text {ref }}-D_{\text {ref }}}$

Equation 2. $x_{\text {cal }}=b_{0}+b_{1} x+b_{2} x^{2}+b_{3} x^{3}$

\section{Study Protocol}

Patients who underwent surgery for colorectal cancer in the Antoni van Leeuwenhoek The Netherlands Cancer Institute (Amsterdam, the Netherlands) and the Slotervaart Medical Centre (Amsterdam, The Netherlands) were included in this ex vivo study. The study was performed under approval of the protocol by the institutional ethics review board.

Immediately after colorectal resection, the entire resected specimen was taken to the pathology department. Cross-sections were cut by the pathologist from the specimen and three tissue samples were obtained; tumor tissue, healthy colon or rectal wall, and (pericolorectal) fat. The cross-sections were placed in a pathology cassette, where they remained during the entire data acquisition. All measurements were performed within 1 hour after specimen resection. Before the hyperspectral measurements, an RGB image was taken of each tissue sample. Next, hyperspectral images were obtained from the tissue samples after which the sampleswere taken backto the pathology department.The samples were processed according to standard protocol in the same pathology cassette, to prevent large tissue deformations. The corresponding H\&E slides were examined by the pathologist, who annotated the various tissue types.

For further data analysis, the digitized annotated slides were registered to the RGB image and the RGB image to the HSI in MATLAB (version 8.5, MathWorks Inc., Natick, Massachusetts, United States), using a nonrigid transformation to overcome the effects of mechanical deformation of the tissue during the standard workflow of tissue processing and staining. Finally, the registered pathology slide was registered to the registered RGB image (Figure 2). Using these registrations, each pixel from the hyperspectral image could be given a histological classification. To create a database of hyperspectral pixels, pixels were manually selected within areas that were defined by the pathologist as absolute certain for a tissue type. About 30 pixels per tissue type per patient were selected when possible. When the surface area of a tissue type was too small to select 30 individual pixels, less pixels were selected. Pixels in the mucosal layer were not taken into account because the mucosa will not be visible during the ultimate surgical application of the technology. Hence, the pixels from the healthy colorectal wall were all in the muscular layer. 


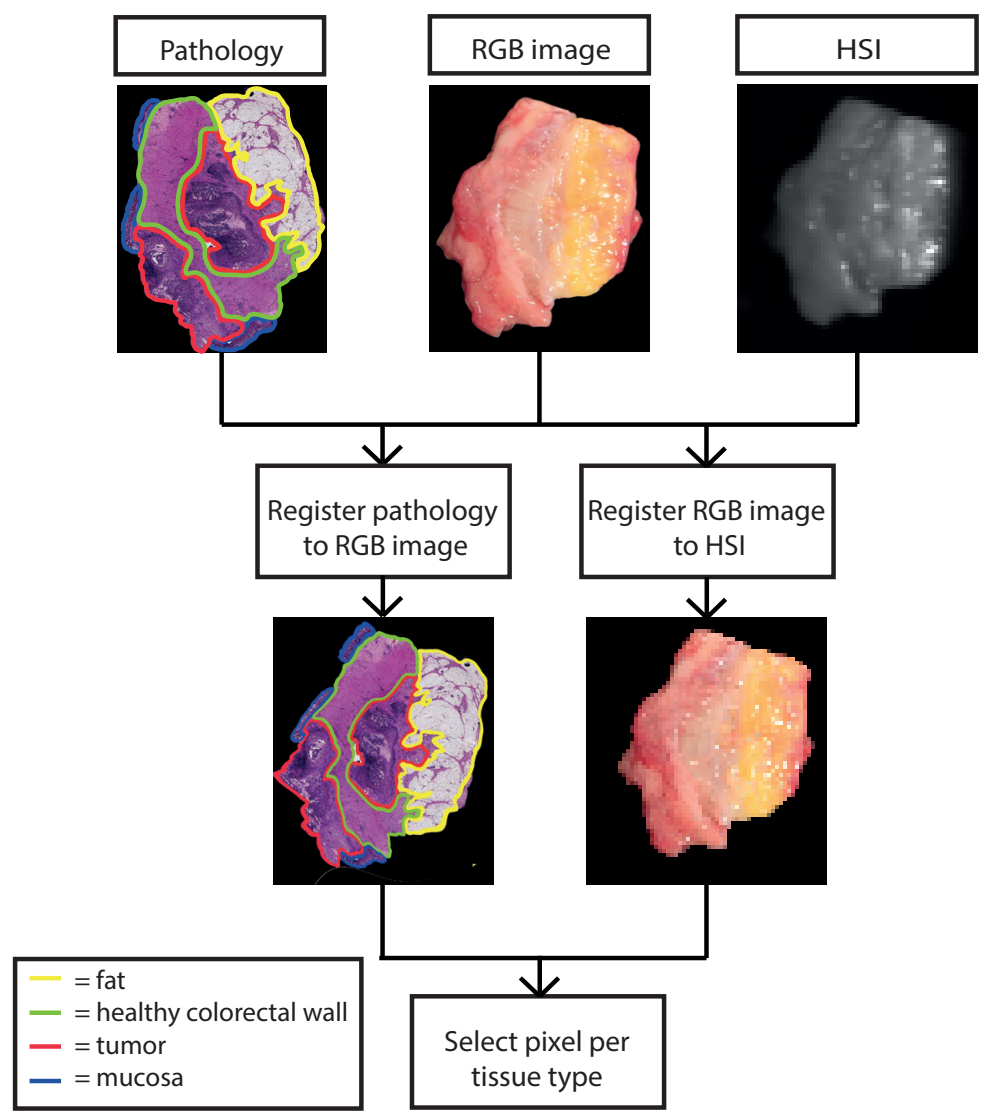

Figure 2. Registration of the $\mathrm{HSI}, \mathrm{RGB}$, and pathology images. In the upper row from left to right, annotated pathology image (yellow $=$ fat, green $=$ healthy colorectal wall, red = tumor, blue = mucosa), RGB image and HSI. The second row from left to right, the annotated pathology image regisered to the RGB image and the RGB image registered to the HSI.

\section{Data Preprocessing}

Preprocessing of the data was performed using a $3.40 \mathrm{GHz}$ Intel Xeon E3-1240 CPU processor and 16 GB RAM and consisted of two steps. First, the spectra were normalized using standard normal variate (SNV) normalization [25]. SNV normalization was performed for each individual spectrum. First, the mean was subtracted from the spectrum after which the spectrum was divided by the standard deviation of the spectrum, see Equation 3. Here, $x_{\text {cor }}$ is the normalized spectrum, $x_{\text {cal }}$ is the calibrated spectrum as given in Equation 1 or Equation 2, and $x_{\text {mean }}$ and $\mathrm{x}_{\text {std }}$ are the mean and standard deviation of $\mathrm{x}_{\text {cal }}$ respectively. This normalization created a zero baseline and a variance equal to one for all spectra:

Equation 3. $\mathrm{x}_{\text {cor }}=\frac{\mathrm{x}_{\text {cal }}-\mathrm{x}_{\text {mean }}}{\mathrm{x}_{\text {std }}}$ 
After combination of the visual and near-infrared images, all outliers caused by specular reflection were removed. Outliers were defined as spectra with an average distance from the mean spectrum of more than three standard deviations, determined over all wavelengths. Next, in order to combine the spectra of the two cameras, the visual images were downsampled. Downsampling was necessary because of the higher resolution of the visual camera compared to the near-infrared camera. A rigid spatial registration was performed obtaining a pixel-to-pixel correlation between the images of the two cameras.

\section{Data Analysis}

Data analysis was performed using the PerClass toolbox (Academic version 5.0, PR Sys design, Delft, The Netherlands) in MATLAB. The data were randomly divided into a training and test set. Per patient, all spectra were assigned to either the training or test set, indicating that spectra from one patient were not split between the training and test set. The training set contained $75 \%$ of the patients and the remaining $25 \%$ was used as a test set. The data contained a hyperspectral image from both the visual and near-infrared camera.

The development of the classification algorithm consisted of three steps. First, feature reduction was applied to the spectra to prevent overfitting of the classifier. For this purpose, k-means clustering was used to determine spectral bands. The clustering is based on the average intensity values of the spectra from the training dataset. For each cluster, if the wavelengths are not continued, the cluster will be divided into multiple spectral bands. The spectral bands were determined once on the training set and were also used on the test set. From these spectral bands, only the mean intensity was used as a feature in the classification algorithm. Second, fat was classified first using a quadratic classifier, which was optimized with a 10-fold cross-validation. The selected optimum in the ROC curve was the point with the lowest mean error. Third, a linear support vector machine (SVM) was used to distinguish the tumor spectra from the healthy colorectal wall spectra. The SVM was also optimized using a 10-fold cross-validation. Classification of the pixels was done based on the probability given by the classifiers. Pixels were assigned to the tissue type with the highest probability. The performance of the classifiers was compared using the area under the ROC curve (AUC), the accuracy, the sensitivity, specificity, and the Matthews correlation coefficient (MCC) [Equation 4]:

Equation 4. $\quad \mathrm{MCC}=\frac{\mathrm{TP} \times \mathrm{TN}-\mathrm{FP} \times \mathrm{FN}}{\sqrt{(\mathrm{TP}+\mathrm{FP})(\mathrm{TP}+\mathrm{FN})(\mathrm{TN}+\mathrm{FP})(\mathrm{TN}+\mathrm{FN})}}$

In Equation 4, TP, TN, FP, and FN are the number of true positives, true negatives, false 
positives, and false negatives, respectively. The MCC returns a value from -1 to +1 , where -1 indicates a total disagreement and +1 indicates a perfect prediction. The accuracy was determined in two different ways. The first accuracy was determined per tissue type and thereafter averaged. The second accuracy was determined per patient and averaged. Finally, to assess the contribution of each camera, the classification was also trained and tested on datasets containing data from only one of the two cameras. The performance of this classification was compared with the classification of the dataset containing data from both cameras, using the ROC curves and the performance measures.

\section{Results}

\section{Patients}

In total, 54 patients were included in this study: 27 men (50\%), 27 women (50\%), with a median age of 65.5 years (IQR: 60 - 73). The samples of 32 patients were imaged with both the near-infrared and the visual camera, 22 additional patients were imaged with only the near-infrared camera. Most of the tumors were located in the colon and sigmoid. One sample showed complete pathological response on preoperative treatment and thus, no tumor tissue could be taken from the sample. Patient and tumor characteristics are described in Table 1.

\section{Data Acquisition and Processing Time}

The obtained tissue samples were first placed under the near-infrared camera and subsequent under the visual camera. Data acquisition times were 20 and 30 seconds for the near-infrared and the visual camera, respectively. In total, data of one patient were acquired in $1 \mathrm{~min}$. The duration of image preprocessing for each tissue sample was 60 seconds in total for both cameras combined for all wavelengths. After training of the classifier, classification of the test data took 2 seconds per patient.

\section{Classification with the Combination of Visual and Near-Infrared Camera}

For the classification of the combination of the visual and near-infrared camera, only the tissue samples scanned with both cameras were included. The dataset of the combined visual and near-infrared camera images contained 2194 spectra, from 32 patients. After outlier removal, 2170 spectra were present in the combined dataset, of which 857 were taken from fat, 563 from muscle, and 750 from tumor. The training set consisted of 24 patients with a total of 1726 spectra. The test set consisted of the remaining 8 patients and 444 spectra. Due to the presence of noise in the lower and upper wavelength range of both cameras, the wavelength ranges of 450 to 950 and 970 to 1600 were selected to 
Table 1. Patient characteristics

\begin{tabular}{lcc}
\hline Characteristics & NIR camera & VIS camera \\
\hline Total no. of patients & 54 & 32 \\
Gender & & 14 \\
Male & 27 & 18 \\
Female & 27 & $66.5(60-71.5)$ \\
Age, median (IQR) & $65.5(60-73)$ & 7 \\
Tumor location & & 12 \\
Cecum & 7 & 11 \\
Colon & 23 & 2 \\
Sigmoid & 21 & 25 \\
Rectum & 3 & 6 \\
Tumor type & & 1 \\
Adenocarcinoma & 46 & \\
Mucinous adenocarcinoma & 7 & 1 \\
Unknown & 1 & 1 \\
Tumor stage & & 4 \\
pT0 & 1 & 16 \\
pT1 & 3 & 10 \\
pT2 & 9 & 26 \\
pT3 & 15 & \\
pT4 & 26 & \\
\hline All & & \\
\hline
\end{tabular}

All patients measured with the VIS camera are also included in the NIR camera measurements 'One patient had a complete response so no tumor type could be determined

analyze for the visual and near-infrared camera, respectively.

As shown in Figure 3, the reflection spectra obtained with the visual camera and the near-infrared camera are not connected after calibration. This is related to differences in optical geometry of the two camera setups. In Figure 3, the large difference in normalized intensity visible at $960 \mathrm{~nm}$ is caused by individual SNV normalization of both cameras before combining the datasets.

Based on the training set, 13 spectral bands were determined, as shown in Figure 3. On the training data, the quadratic classifier obtained an MCC, AUC, accuracy, sensitivity, and specificity of 1.00 to separate fat from healthy and tumor. The SVM applied on the training dataset, to distinguish tumor from muscle, provided an MCC of 0.83, an AUC of 0.98, an accuracy of 0.91 , and the sensitivity and specificity were 0.93 and 0.90 , respectively. The accuracy of combination of the quadratic classifier and the SVM on the training dataset was 0.94 (STD =0.04) when assessed on the tissue types. When determined per patient and averaged, a training accuracy of $0.94($ STD $=0.13$ ) was obtained.

The results of the combination of the quadratic classifier and the SVM on the test dataset are shown in Table 2. The accuracy determined per tissue type and averaged on the test dataset was $0.88(S T D=0.13)$. The accuracy calculated per patient and thereafter averaged was $0.93(\mathrm{STD}=0.12)$. 


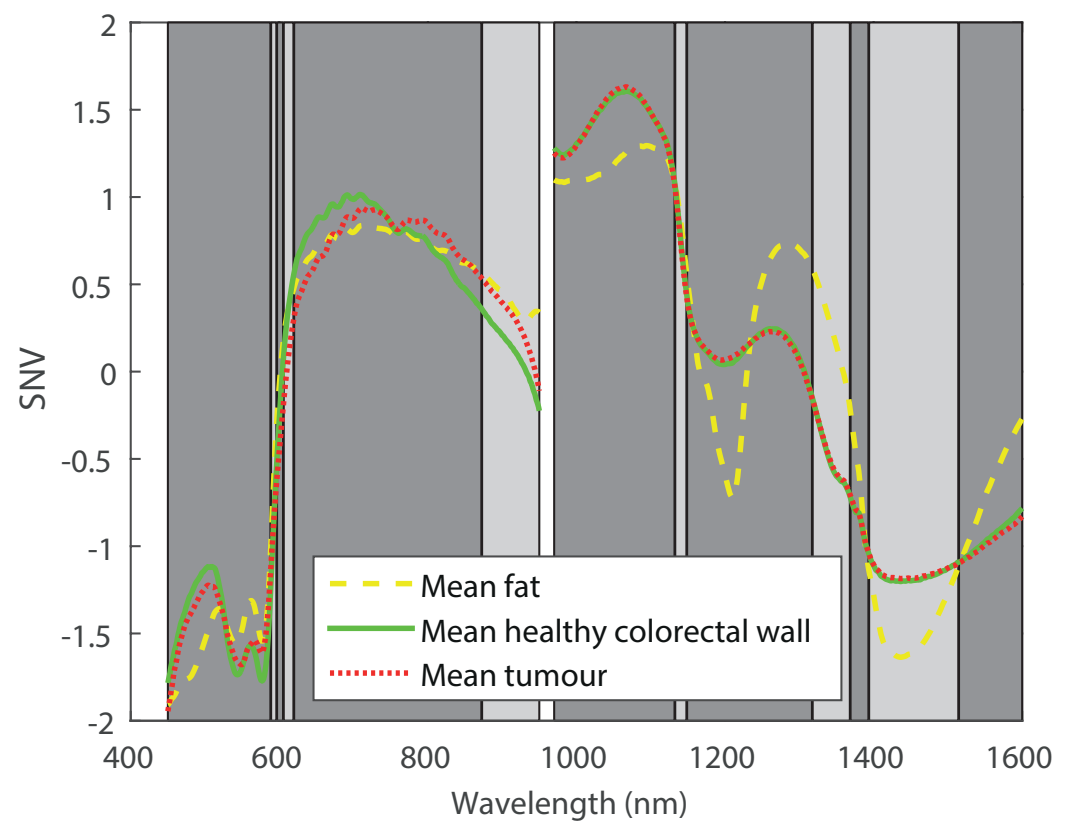

Figure 3. Spectral bands determined for the dataset with the combination of visual and near-infrared camera images are shown together with the mean spectra of fat (yellow), healthy colorectal wall (green), and tumor (red). The 13 spectral bands all have a different gray value and are separated by black vertical lines. Between 950 and $970 \mathrm{~nm}$, a gap is shown in the data. This region is not covered by the cameras.

In Figure 4, the results of the classification of all pixels in a hyperspectral image of one patient from the test set are shown. The different colors represent different tissue types. The certainty of the classification, based on the probability, is shown by the intensity of the color. The more intense the color is, the higher the certainty of the classifier is for this classification.

Table 2. Results of the combined classifiers on the test dataset with a combination of visual and nearinfrared camera images. The mean accuracy averaged over the tissue types was $0.88(S T D=0.13)$ and over the patients was $0.93(\mathrm{STD}=0.12)$.

\begin{tabular}{lcccccr} 
& & \multicolumn{2}{c}{ Decision based on hyperspectral data } & \multicolumn{2}{c}{ Total } \\
\cline { 3 - 5 } & & Fat & Muscle & Tumor & 17 \\
\hline Gold standard & Fat & 187 & 0 & 0 & 4 & $\mathbf{1 1 5}$ \\
& Muscle & 30 & 81 & 9 & 133 & $\mathbf{1 4 2}$ \\
& Tumor & 0 & $\mathbf{9 0}$ & $\mathbf{1 3 7}$ & $\mathbf{4 4 4}$
\end{tabular}




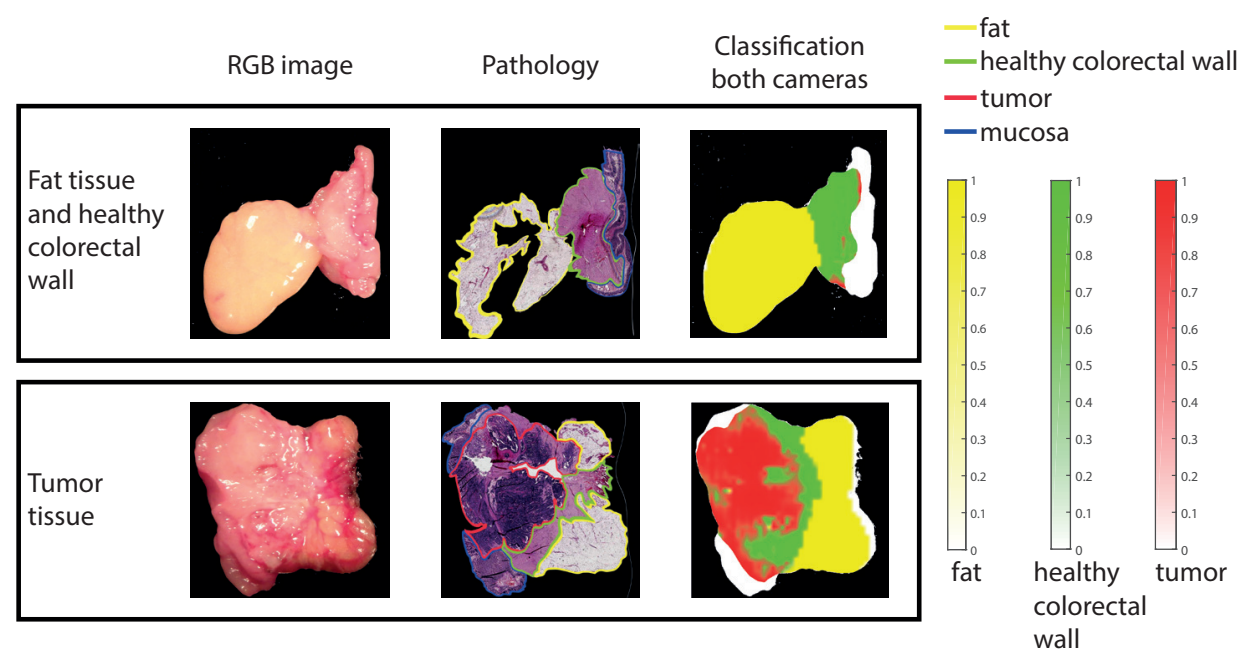

Figure 4. Classification of the tissue samples of one patient from the test set of the combined data set of the visual and near-infrared camera. In the first column, the RGB image of each tissue sample is shown. The second column shows the registered annotated pathology image (yellow $=$ fat, green $=$ muscle or healthy colorectal wall, red = tumor, blue = mucosa). In the third column, the classification based on the visual and near-infrared spectra is shown projected on the binary mask of the RGB image (yellow = fat, green $=$ muscle of healthy colorectal wall, red = tumor). The first row shows the healthy tissue including fat and healthy colorectal wall. The second row shows the tumor tissue sample. Tissue annotated by the pathologist as mucosa (blue) is not classified and is shown white in the third column.

\section{Classification with a Single Camera}

The classification of fat, healthy colon or rectal wall, and tumor was also performed on the datasets including only one of the two cameras. The dataset with only spectra from the near-infrared camera contained 54 patients and 4352 spectra of which 1690 were measured in fat, 1251 in the muscular layer of healthy colon or rectal wall, and 1411 in tumor. After removal of the outliers, 4309 spectra remained (1676 fat, 1232 muscle, and 1401 tumor). For the training set, 41 patients were randomly selected with a total of 3241 spectra. The test set contained 13 patients and 1068 spectra.

For the images of the visual camera, the same pixels were used as selected for the images of the near-infrared camera. A total of 2194 spectra, from 32 patients, were included in this dataset. From the spectra, 866 were measured in fat, 569 in muscle, and 759 in tumor. After removal of the outliers, 2164 spectra remained (854 fat, 560 muscle, and 750 tumor). The training set included 24 patients with 1723 spectra, and the remaining 8 patients were included in the test set, which contained 441 spectra. After spectral bands were extracted from the datasets, the quadratic classifiers were trained and ROC curves for both datasets were obtained. In Figure 5, the ROC curves of both classifiers are shown together with the ROC curve of the classifier created with the combined dataset. This shows a slightly worse performance for the dataset with 
only visual camera images compared to the dataset with only near-infrared images or the dataset including image of both cameras. This was also seen in the performance measures, with an MCC of 0.90 for the dataset with only visual camera images, and an MCC of 0.99 and 1.00 for the dataset with only near-infrared camera images and the combined dataset, respectively. All other performance measures showed the same trend.

In Figure 6, the ROC curves of the SVMs are shown for the three training datasets. Here, again, the dataset containing only visual camera images showed the worst performance. However, there is a clear difference between the dataset containing only near-infrared camera images and the dataset containing images of both cameras, where the latter outperformed the first. A summary of the performance measures of the SVM is shown in Table 3. The same trend is shown for all performance of the three datasets; the dataset including only visual camera images performed worst and the combined dataset performed best. The results of the test set of the two datasets containing data of only one of the two cameras resulted in an accuracy for determining the tissue types of 0.67 (STD $=0.19)$ and $0.83(S T D=0.12)$ for the visual camera data and near-infrared camera data, respectively. The accuracy calculated per patient and averaged was 0.71 (STD $=0.19$ ) for the visual camera data and 0.83 (STD =0.14) for the near-infrared camera data. The classifiers created were also used to classify the spectra from each pixel of entire hyperspectral images of one of the patients from the test set that was imaged by both cameras. In Figure 7, the result of this classification is shown for all three classifications.

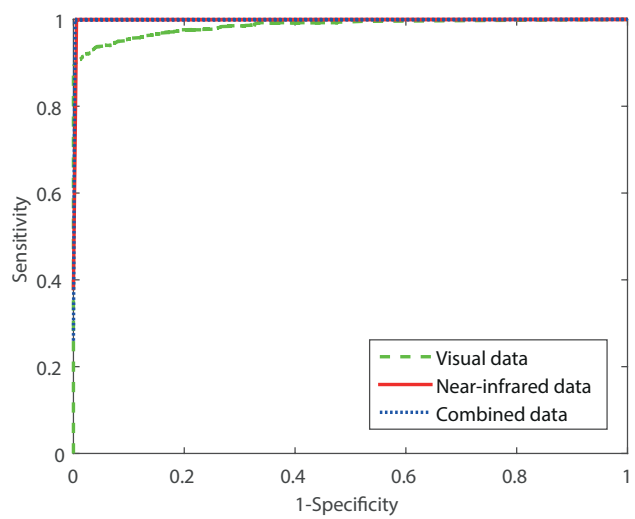

Figure 5. ROC curves of the training results of the quadratic classifier distinguishing fat from all other tissue types. The three datasets are shown as the visual camera (green), near-infrared camera (red), and the combination of the visual and near-infrared camera (blue).

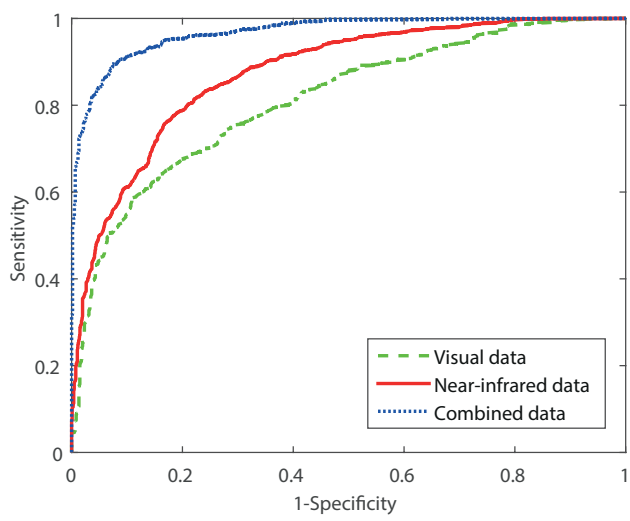

Figure 6. ROC curves of the training results of the SVMs distinguishing tumor from healthy colorectal tissue. The three datasets are shown as the visual camera (green), near-infrared camera (red), and the combination of the visual and near-infrared camera (blue). 
Table 3. Performance measures for the SVM from the three training datasets.

\begin{tabular}{|c|c|c|c|c|}
\hline Performance measure & Visual camera & \multicolumn{2}{|c|}{ Near-infrared camera } & Combined dataset \\
\hline MCC & 0.50 & \multicolumn{2}{|c|}{0.59} & 0.83 \\
\hline AUC & 0.81 & \multicolumn{2}{|c|}{0.87} & 0.98 \\
\hline Accuracy & 0.74 & \multicolumn{2}{|c|}{0.80} & 0.91 \\
\hline Sensitivity & 0.77 & \multicolumn{2}{|c|}{0.78} & 0.93 \\
\hline Specificity & 0.74 & \multicolumn{2}{|c|}{0.81} & 0.90 \\
\hline RGB image & Pathology & $\begin{array}{l}\text { Classification } \\
\text { visual camera }\end{array}$ & $\begin{array}{r}\text { Classific } \\
\text { near-in } \\
\text { cam } \\
\end{array}$ & $\begin{array}{l}\text { Classification } \\
\text { both cameras }\end{array}$ \\
\hline Fat tissue & & & & \\
\hline
\end{tabular}
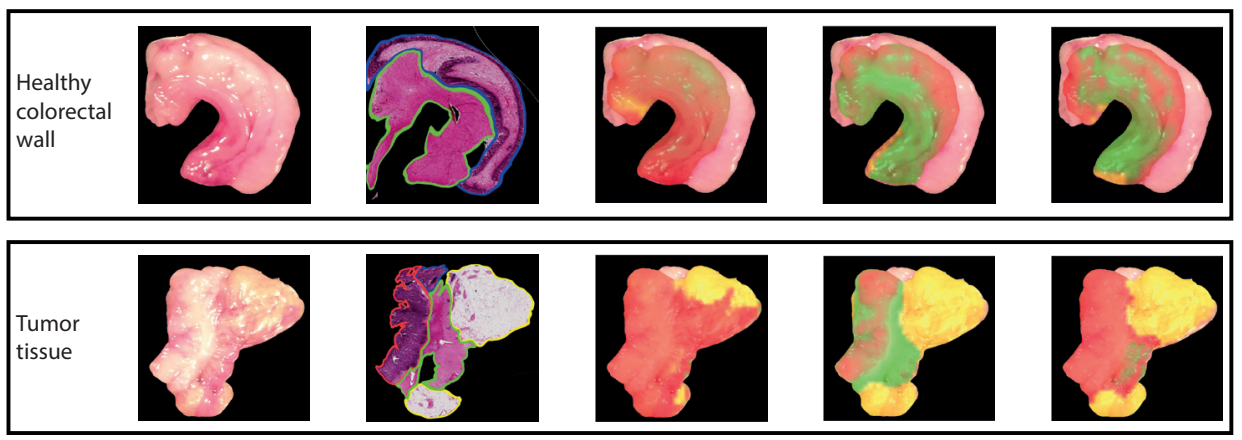

- fat, - healthy colorectal wall, — tumor, - mucosa

Figure 7. Classification of the tissue samples of one patient from the test dataset of the combined dataset. In the first column, the RGB image of each tissue sample is shown. The second column shows the registered annotated pathology H\&E image (yellow $=$ fat, green = muscle or healthy colorectal wall, red = tumor, blue = mucosa). In the third to fifth column from the classification based on the visual image only, the classification based on the near-infrared image only and the classification based on the combined visual and near-infrared image are shown, respectively, projected on the binary mask of the RGB image (yellow $=$ fat, green = muscle of healthy colorectal wall, red = tumor). From top to bottom, fat, healthy colorectal wall, and tumor tissue are shown. Tissue annotated as mucosa (blue) by the pathologist is not classified and shown as white in the third to fifth column.

\section{Discussion}

In this study, the potential added value of HSI for fast tissue classification during colorectal cancer surgery was examined. As a first step, an ex vivo study was designed in which tissue samples from colorectal cancer surgery were imaged with two hyperspectral cameras. One camera obtained images in the visual wavelength range (400 to $1000 \mathrm{~nm})$, and the second camera obtained images in the near-infrared wavelength range (900 to $1700 \mathrm{~nm}$ ). HSI allowed accurate discrimination of fat, healthy colon or rectal wall, and tumor tissue, with an accuracy of $0.88(S T D=0.13)$ for the combination 
of the visual and near-infrared camera images.

Current literature of HSI in colorectal cancer was mainly focused on the classification of H\&E pathology slides or classification of tissue during endoscopy [15-20, 22]. For the first application, hyperspectral images were made of H\&E pathology slides to obtain objective classification into healthy or malignant tissue of colon biopsies [15-19]. This application is far from the goal of the current study as the current study is focused on near realtime imaging during surgery. For the second application, a hyperspectral camera was combined with an endoscope, to obtain hyperspectral images during endoscopy. The study by Kumashiro et al. obtained in vivo hyperspectral data during colonoscopy and was able to distinguish tumor from healthy mucosa with a sensitivity of 0.73 and a specificity of 0.82 , using Pearson correlation analysis [22]. The study by Han et al. obtained better results with an accuracy of 0.94 and a sensitivity and specificity of 0.97 and 0.91 , respectively [21]. This study used hyperspectral images in the spectral range from 405 to $665 \mathrm{~nm}$, so only the visual wavelength range. These results are better than the results shown in the current study for the dataset including only the visual camera images. The main difference between the two studies and the current study is the location of the measurements. Han et al. and Kumashiro et al. performed measurements of the lumen of the colon during endoscopy, whereas the current study focused on the surgical application and performed measurements from the surface of the colon [21-22].

In a previous study, we showed the possibility to distinguish the three tissue types - fat, healthy colon or rectal wall, and tumor - using fiberoptic diffuse reflectance spectroscopy (DRS) in a surgical setting [26]. The information obtained in fiberoptic DRS is very similar to the information obtained with a hyperspectral camera system. Therefore, it is not surprising that the results obtained in the current study are comparable to the results obtained in the previous study using DRS. However, the accuracy of the current study is slightly less compared to the accuracy obtained with the DRS measurements (accuracy $=0.95, \mathrm{STD}=0.03$ ). An explanation for this difference might be due to the correlation with the gold standard, histology, which is less challenging for the fiberoptic point measurements performed in the previous study. In the testing of the combination of the two cameras, 30 muscle spectra of one particular patient were classified as fat. These misclassifications are most likely due to a fault in the registration between the hyperspectral images and the histology in this specific patient. The influence of this one patient can be seen in the accuracy determined per patient, which is 0.93 and similar to the accuracy obtained in the DRS study.

For the evaluation of the performance of the classifiers, six different performance measures were used. Of these parameters, the MCC and AUC are most accurate in the 
current study, because these parameters are relatively insensitive to the effect of an imbalanced dataset [27]. However, for the combination of the quadratic classifier and the SVM, the MCC and AUC cannot be used, because both measures only account for a two class problem. For the quadratic classifier, no large differences are shown between the performance measures for the three datasets. However, the performance measures of the SVM did show a difference. The combination of the two cameras clearly outperforms the datasets using only one of the two cameras (Table 3). For the combination of the quadratic classifier and the SVM, the accuracies can be compared. Here, the combination of the two cameras outperforms the datasets with data from only one of the two cameras.

Comparing the two cameras, the near-infrared camera slightly outperforms the visual camera, with a MCC of 0.59 and 0.50, respectively. In Figure 3, only the average curves are shown per tissue type. Although the difference between the average spectra of colon and tumor tissue is bigger in the visual part of the spectrum, also the standard deviation (not shown) is higher in the visual part compared to the near-infrared part. Furthermore, the SVM used for the classification of healthy colon and tumor does not take into account each feature individually but uses the combination of features to find the optimal hyperplane, which differentiates the two classes. In accordance, the combination of the features from the near-infrared part of the spectrum might give a better result than the combination of the features from the visual part of the spectrum. It is hard to visualize these distinctive differences in the near-infrared part of the spectrum. In line with the explanation above, as mentioned before, the MCC value for the discrimination between healthy and tumor was slightly higher in the nearinfrared part of the spectrum, the combination of the near-infrared and visual part of the spectrum gives the best results because of the combination of the features that can be made. This increases the accuracy with 0.21 to 0.88 .

For the translation of the technique to an in vivo setting, where it will be used during surgery, the large dependence on the near-infrared wavelength ranges is favorable. The main difference between an ex vivo setting and an in vivo setting is the presence of blood. For oxygenated and deoxygenated blood, the main absorption bands are located in the visual wavelength range. Therefore, blood absorption will have no influence in the near-infrared wavelength range [28]. The influence of blood in the translation to an in vivo setting using a classification method based mostly on the near-infrared wavelength range will thus be small [29].

Previous work in our group showed good results in combining a quadratic classifier and a linear SVM in tissue identification in colorectal cancer using fiberoptic DRS [26]. In the current study also, two different classifiers to distinguish between fat, healthy 
colorectal wall, and tumor are used. First, a quadratic classifier was used to classify fat; second, a linear SVM was used to distinguish healthy colorectal wall from tumor. Because fat was first classified with the quadratic classifier, a binary task was left for the SVM. Therefore, a simple linear SVM could be used to distinguish healthy colorectal wall from tumor tissue. To perform a classification of three tissue types using only SVMs, a one-against-one or one-against-all classification should be performed. This will result in a combination of at least three SVMs, which will be a more complex classifier compared to the combination currently used. The more complex the classifier is, the more prone the classifier is to overfitting. Because the classification of fat was easy to perform, a more simple approach could be used in the form of a two-step classification, combing a quadratic classifier with a single SVM.

The classifiers created in this study were only based on the spectral features. However, because 2-D images were obtained with the hyperspectral cameras, there is also an option to use spatial and textural properties of the images to classify the pixels. In the current study, this option was not used because the spatial and textural properties during surgery will be very different compared to the properties, which would have been obtained in this study. However, for future studies, textural properties may be taken into account and could be used to further improve the current classification results.

In this ex-vivo study, the pathologist cut cross-section slices of the tumor and colorectal wall provide a large surface area of tumor and healthy tissue. This method was chosen to obtain a sufficient amount of data to create a reliable classification. In a surgical setting, these large surfaces of tumor will not be seen. Rectal tumors start developing in the mucosa and grow through the muscle layer into the surrounding mesorectal fat when becoming more advanced. In contrast to the large volume of tumor present in the lumen and wall of the rectum, smaller volumes of the tumor will be present in the mesorectal fat and possibly in the resection surface created by the surgeon. So, the main question for future research should be whether the current classification will still be able to detect an area of tumor tissue, which is much smaller compared to the cross-section slices and mainly surrounded by healthy tissue. So, as a next step toward in vivo use, the entire resected specimens should be imaged with HSI to validate the current accuracy in a more realistic setting.

To be able to perform HSI during surgery, some technical changes need to be made. The currently used set-up is a push-broom camera, where the samples are scanned by moving through the imaging line of the camera. In an in vivo setting, especially during laparoscopic surgery, this will not be possible. Therefore, a snapshot multispectral camera should be used, which can be attached to a laparoscopic 
system. In a multispectral camera, a limited number of wavelengths can be measured. These wavelengths should be chosen based on previous research. Therefore, further research should be performed on the selection of the most important wavelengths to distinguish tumor from healthy surrounding tissue. Using a snapshot camera will reduce the data acquisition time compared to the current setup, from $1 \mathrm{~min}$ to $1 \mathrm{~s}$. Moreover, the preprocessing time of the data will decrease because of the limited number of wavelengths acquired. Therefore, when the current set-up is transformed into a set-up that can be used in vivo, real-time tissue classification will be possible. Furthermore, in the in vivo setting, the measurements will be less controlled compared to the current study. For example, the illumination of the tissue will be variable during surgery. Moreover, specular reflection and glare will be present. These issues should be taken into consideration before starting an in vivo study. Finally, when performing measurements in vivo, a real-time classification should be available. The current classification method would allow such real-time use.

\section{Conclusion}

In this ex vivo study, fat, healthy colorectal wall, and tumor tissue could be distinguished using $\mathrm{HSI}$ with an accuracy of $0.88(\mathrm{STD}=0.13)$. When the accuracy is determined per patient, a mean accuracy of $0.93($ STD $=0.12$ ) was obtained. Two hyperspectral cameras were used, one in the visual wavelength range and one in the near-infrared wavelength range. Using only one of the two cameras decreased the accuracy for the visual and near-infrared camera. The results of this study show the potential of using HSI during colorectal surgery to increase the number of radical resections. Future research should be focused on imaging of entire specimen and the translation of the technique to an intraoperative setting. This should result in a technique that provides accurate realtime tissue classification during laparoscopic colorectal cancer surgery. 


\section{References}

1. Ferlay J, Soerjomataram I, Dikshit R, et al. Cancer incidence and mortality worldwide: Sources, methods and major patterns in GLOBOCAN 2012. Int J Cancer. 2015;136(5):E359-E386. doi:10.1002/ ijc.29210.

2. Rabeneck L, Horton S, Zauber AG, Earle C. Cancer: Disease Control Priorities. In: Disease Control Priorities 3rd Edition. ; 2015:101-119. doi:10.1017/CBO9781107415324.004.

3. Pascual M, Salvans S, Pera M. Laparoscopic colorectal surgery: Current status and implementation of the latest technological innovations. World J Gastroenterol. 2016;22(2):704-717. doi:10.3748/wjg. v22.i2.704.

4. Ottermo M V, Øvstedal M, Langø T, et al. The Role of Tactile Feedback in Laparoscopic Surgery. Surg Laparosc Endosc Percutaneous Tech. 2006;16(6). https://journals.Iww.com/surgical-laparoscopy/ Fulltext/2006/12000/The_Role_of_Tactile_Feedback_in_Laparoscopic.4.aspx.

5. Schostek S, Schurr MO, Buess GF. Review on aspects of artificial tactile feedback in laparoscopic surgery. Med Eng Phys. 2009;31(8):887-898. doi:10.1016/J.MEDENGPHY.2009.06.003.

6. Lu G, Fei B. Medical hyperspectral imaging: a review. J Biomed Opt. 2014;19(1):10901. doi:10.1117/1 JBO.19.1.010901.

7. Benavides JM, Chang S, Park SY, et al. Multispectral digital colposcopy for in vivo detection of cervical cancer. Opt Express. 2003;11(10):1223-1236. doi:10.1364/OE.11.001223.

8. Boucheron LE, Bi Z, Harvey NR, Manjunath BS, Rimm DL. Utility of multispectral imaging for nuclear classification of routine clinical histopathology imagery. BMC Cell Biol. 2007;8(Suppl 1):S8-S8. doi:10.1186/1471-2121-8-S1-S8.

9. Hattery D, Hassan M, Demos S, Gandjbakhche A. Hyperspectral imaging of Kaposi's Sarcoma for disease assessment and treatment monitoring. In: Applied Imagery Pattern Recognition Workshop, 2002. Proceedings. ; 2002:124-130. doi:10.1109/AIPR.2002.1182265.

10. Liu Z, Wang H, Li Q. Tongue Tumor Detection in Medical Hyperspectral Images. Sensors (Basel). 2012;12(1):162-174. doi:10.3390/s120100162.

11. Fei B, Lu G, Wang X, et al. Label-free reflectance hyperspectral imaging for tumor margin assessment: a pilot study on surgical specimens of cancer patients. J Biomed Opt. 2017;22(8):1. doi:10.1117/1. JBO.22.8.086009.

12. Lu G, Little J V, Wang X, et al. Detection of Head and Neck Cancer in Surgical Specimens Using Quantitative Hyperspectral Imaging. Clin Cancer Res. 2017;23(18):5426 LP-5436. doi:10.1158/10780432.CCR-17-0906.

13. Akbari H, Uto K, Kosugi Y, Kojima K, Tanaka N. Cancer detection using infrared hyperspectral imaging. Cancer Sci. 2011;102(4):852-857. doi:10.1111/j.1349-7006.2011.01849.x.

14. Kiyotoki S, Nishikawa J, Okamoto T, et al. New method for detection of gastric cancer by hyperspectral imaging: a pilot study. 2013;18:26010-26017. https://doi.org/10.1117/1.JBO.18.2.026010.

15. Masood K, Rajpoot NM (Nasir M. Spatial analysis for colon biopsy classification from hyperspectral imagery. 2008. http://wrap.warwick.ac.uk/37082/\#.WmdDFQM7u8w.mendeley. Accessed January 23, 2018.

16. Masood K, Rajpoot NM (Nasir M. Classification of colon biopsy samples by spatial analysis of a single spectral band from its hyperspectral cube. 2007. http://wrap.warwick.ac.uk/61638/\#. WmdFQdBrhPY.mendeley. Accessed January 23, 2018.

17. Rajpoot K, Rajpoot N. SVM Optimization for Hyperspectral Colon Tissue Cell Classification. In: Barillot C, Haynor DR, Hellier P, eds. Medical Image Computing and Computer-Assisted Intervention -- MICCAI 2004. Berlin, Heidelberg: Springer Berlin Heidelberg; 2004:829-837.

18. Rathore S, Hussain M, Ali A, Khan A. A Recent Survey on Colon Cancer Detection Techniques. IEEE/ ACM Trans Comput Biol Bioinforma. 2013;10(3):545-563. doi:10.1109/TCBB.2013.84.

19. Maggioni M, Davis GL, Warner FJ, et al. Hyperspectral microscopic analysis of normal, benign and carcinoma microarray tissue sections. In: Alfano RR, Katz A, eds. Vol 6091. International Society for Optics and Photonics; 2006:60910l. doi:10.1117/12.646078. 
20. Claridge E, Hidović-Rowe D. Model Based Inversion for Deriving Maps of Histological Parameters Characteristic of Cancer From Ex-Vivo Multispectral Images of the Colon. IEEE Trans Med Imaging. 2014;33(4):822-835. doi:10.1109/TMI.2013.2290697.

21. Han Z, Zhang A, Wang X, Sun Z, Wang MD, Xie T. In vivo use of hyperspectral imaging to develop a noncontact endoscopic diagnosis support system for malignant colorectal tumors. 2016;21:1600116008. http://dx.doi.org/10.1117/1.JBO.21.1.016001.

22. Kumashiro R, Konishi K, Chiba T, et al. Integrated Endoscopic System Based on Optical Imaging and Hyperspectral Data Analysis for Colorectal Cancer Detection. Anticancer Res. 2016;36(8):3925-3932. http://ar.iiarjournals.org/content/36/8/3925.abstract.

23. OOINLCorrect Loading Non-Linearity Correction Coefficients Instructions. Dunedin; 2012. https:// oceanoptics.com/wp-content/uploads/OOINLCorrect-Linearity-Coeff-Proc.pdf.

24. Burger J, Geladi P. Hyperspectral NIR image regression part I: calibration and correction. J Chemom. 2005;19(5-7):355-363. doi:10.1002/cem.938.

25. Rinnan $\AA$, Berg F van den, Engelsen SB. Review of the most common pre-processing techniques for near-infrared spectra. TrAC Trends Anal Chem. 2009;28(10):1201-1222. doi:10.1016/J. TRAC.2009.07.007.

26. Baltussen EJM, Snæbjörnsson P, Koning SGB de, et al. Diffuse reflectance spectroscopy as a tool for real-time tissue assessment during colorectal cancer surgery. 2017;22:106014-106016. https://doi. org/10.1117/1.JBO.22.10.106014.

27. Boughorbel S, Jarray F, El-Anbari M. Optimal classifier for imbalanced data using Matthews Correlation Coefficient metric. Zou Q, ed. PLoS One. 2017;12(6):e0177678. doi:10.1371/journal. pone.0177678.

28. Bydlon TM, Nachabé R, Ramanujam N, Sterenborg HJCM, Hendriks BHW. Chromophore based analyses of steady-state diffuse reflectance spectroscopy: current status and perspectives for clinical adoption. J Biophotonics. 2015;8(1-2):9-24. doi:10.1002/jbio.201300198.

29. Spliethoff JW, Prevoo W, Meier MAJ, et al. Real-time in vivo tissue characterization with diffuse reflectance spectroscopy during transthoracic lung biopsy: a clinical feasibility study. Clin Cancer Res. 2015;22(2):357-365. doi:10.1158/1078-0432.ccr-15-0807. 



\section{Chapter 6}

\section{Association of image-guided navigation with complete resection in patients with locally advanced primary and recurrent rectal cancer}

Esther N.D. Kok Ruben van Veen

Harald C. Groen Wouter J. Heerink Nikie J. Hoetjes Erik van Werkhoven Geerard L. Beets Arend G.J. Aalbers Koert F.D. Kuhlmann Jasper Nijkamp Theo J.M. Ruers 


\section{Abstract}

Importance: The percentage of tumor-positive surgical resection margins in patients treated for locally advanced primary or recurrent rectal cancer is high. Image-guided navigation may improve complete resection rates.

Objectives: To ascertain whether image-guided navigation during rectal cancer surgery improves complete resection rates compared with rectal cancer surgery without navigation.

Design, Setting, and Participants: This prospective single-center nonrandomized controlled trial included patients with locally advanced primary or recurrent rectal cancer operated with image-guided navigation between February 2016 and September 2019, at a tertiary referral hospital. The clinical results were compared with results of patients from a historical cohort, who received rectal cancer surgery without imageguided navigation between Januari 2009 and December 2015.

Main Outcomes and Measurements: The primary endpoint of this study was the complete resection rate, measured by the amount of tumor negative resection margins. Secondary outcomes were safety and usability of the system.

Results: In this prospective study, 33 patients with advanced rectal cancer were included (23 men (69.7\%); median age: 61 years (IQR: 55.0 - 69.0)). After resection of locally advanced tumors, a radical resection (R0) was achieved in 93\% (13/14 patients) and after rectal resection of recurrent rectal cancer in $79 \%$ (15/19 patients) of the patients. No navigation-related complications occurred before or during surgery. In the historical cohort, 142 patients who underwent resection without image-guided navigation were included (95 men (66.9\%); median age: 64 years (IQR: 55.0 - 70.0)). In these patients, an R0 resection was accomplished in 84\% (85/101 patients) for locally advanced rectal cancer and in 49\% (20/41 patients) for recurrent rectal cancer. A significant difference was found between the navigation and historical cohort after recurrent rectal cancer surgery $(p=0.047)$. Surgeons stated that the navigation system improved decisiveness and helped with tumor localization.

Conclusion and Relevance: This study suggest that image-guided navigation used during rectal cancer surgery is safe and intuitive, and may improve tumor-free resection margin rates in recurrent rectal cancer surgery. 


\section{Introduction}

Surgery is the main treatment for patients with rectal cancer. Surgical resection can be challenging, especially in patients with primary advanced disease where the mesorectal fascia is threatened and tissue planes are disrupted by tumor ingrowth, fibrosis or radiation effects. Despite improved neoadjuvant treatment and refinement of surgical techniques, the proportion of tumor positive resection margins remains 10$15 \%[1,2]$. Previous studies have shown that a positive resection margin is associated with high local recurrence rates and poorer survival [3, 4]. Surgery in patients with local recurrence is even more challenging because of fibrotic scar tissue of the previous operation. Rectal recurrence is often accompanied by tumor ingrowth in surrounding tissue and organs, requiring extensive surgical procedures like pelvic exenteration. These procedures are associated with high tumor positive resection margins ranging from $38 \%$ to $62 \%$, major complications rates from $32 \%$ to $60 \%$ and 30 -day mortality rates from $0 \%$ to $16 \%$ [5-11].

A possible means to improve the safety and effectiveness of surgery for locally advanced and recurrent rectal cancer could be image-guided navigation. This technique provides surgeons with real-time intraoperative feedback on the position of surgical instrumentation relative to the intraoperative anatomy, including tumor borders. It could improve complete removal of tumors without damaging the surrounding healthy structures [12].

In an investigation of whether image-guided navigation was possible in more advanced rectal surgery, a novel electromagnetic (EM) surgical navigation system was developed for pelvic malignancies. This in-house developed navigation system has shown to be safe, feasible and accurate [13]. In the current study, we evauated the clinical benefit of this image-guided navigation system. We hypothesized that surgical navigation during surgery for advanced and recurrent rectal cancer would allow for full use of pre-operative imaging during the surgical procedure and would improve surgical outcome by a higher complete resection rate compared with results in a historical cohort. Moreover, we examined the safety and usability of this intraoperative technique.

\section{Methods}

\section{Study design and patient selection}

We conducted a prospective, single-center nonrandomized controlled trial to evaluate the use of an electromagnetic (EM) navigation system during abdominal surgery. The analysis presented here consists of the cohort between February 2016 and September 
2019. Inclusion criteria were: patients diagnosed with locally advanced primary or recurrent rectal cancer in which surgeons judged radical resection challenging, $\geq 18$ years old, suitable for contrast-enhanced CT scanning and scheduled for abdominal surgery in our hospital. Patients with metal pelvic implants or with a pacemaker were excluded because of the negative influence on the preoperative imaging. Locally advanced rectal cancer was defined as T3 or T4 tumors extending close to $(<2 \mathrm{~mm})$ or invading the mesorectal fascia, based on rectal MRI. Recurrent rectal cancer was defined as cancer that recurred in the pelvic area after earlier treatment. This study was approved by the institutional review board (NL43553.031.13) of the Netherlands Cancer Institute - Antoni van Leeuwenhoek, Amsterdam, The Netherlands. All patients provided written informed consent. The study is registered at www.trialregister.nl (NTR7184).

\section{Navigation system}

Full details of the navigation setup have been published elsewhere [13]. Briefly, a patient-specific 3D model was created based on preoperative imaging (CT, MRI). In the operating room, a cone-beam $\mathrm{CT}$ (CBCT) scan was acquired of the patient in surgical position. The intraoperative images were registered to the preoperative images based on the bony structures. An EM tracking system constantly linked the preoperative images and 3D model to the intraoperative position of the patient by EM sensors affixed on the skin. During surgery, an EM tracked pointer was available to validate

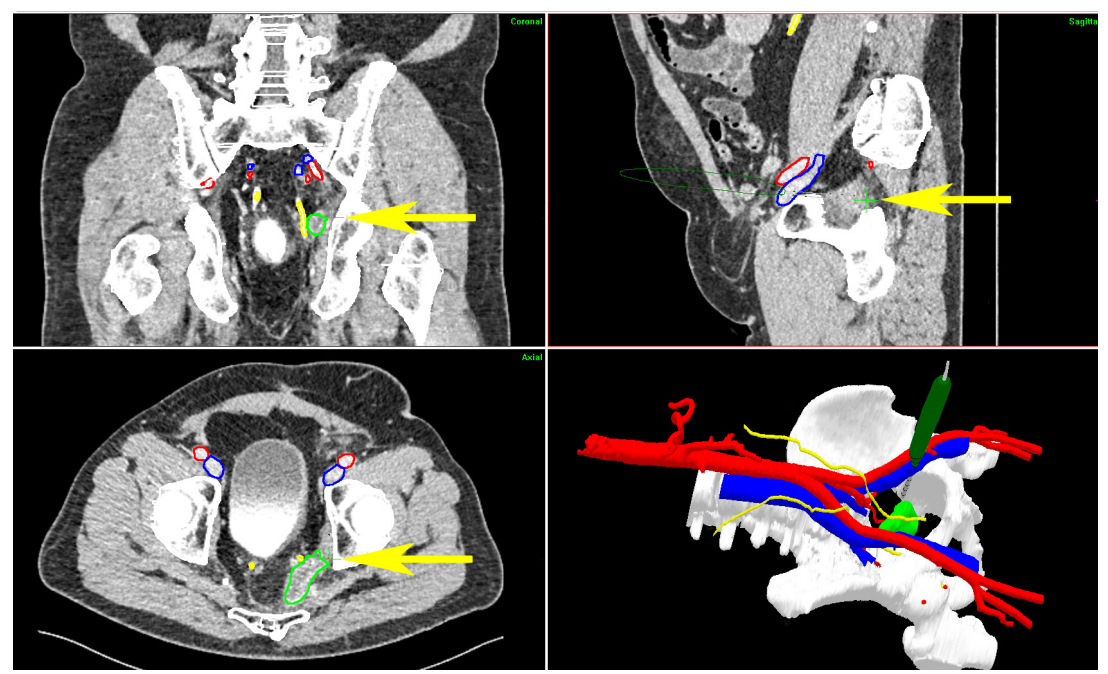

Figure 1. Typical example of the surgical navigation user interface during recurrent rectal cancer surgery (tumor segmented in green, ureters in yellow, vessels in blue/red). The planning CT scan with segmentations (in coronal, sagittal and axial plane) and the 3D model are shown. The surgical pointer is shown in the 3D model and orthogonal views (highlighted with arrows). 
the registration accuracy and to assess the anatomy and tumor location (Figure 1).

\section{Historical controls}

For the historical cohort, patients with locally advanced primary or recurrent rectal cancer, age $\geq 18$ years old, who received rectal surgery without the navigation system were eligible. Clinical data were collected retrospectively from medical records and anonymized. In total, 142 patients met the inclusion criteria and received surgery between January 1,2009, and December 31,2015. The historical group was well balanced with the prospective group, no significant differences in baseline characteristics were found. Permission to collect the retrospective data was granted by the Institutional Review Board (IRBd19193).

\section{Study endpoints}

The primary endpoint of this study was the complete resection rate, measured by the number of tumor negative resection margins. After primary rectal resection, tumor $>1$ $\mathrm{mm}$ of the circumferential resection margin was defined as radical (R0). After recurrent rectal resection, the resection margin was defined as radical (R0) when no tumor cells reached the border of the inked resection specimen.

The secondary endpoints were safety and usability of the system. Safety of the system was evaluated by the number of navigation-related surgical adverse events. Intraoperative surgical complications not related to the navigation system were also reported. Usability of the system was evaluated with questionnaires filled in by the participating surgeons after each procedure. Questionnaires consisted of 2 parts. First, the surgical usability was tested using the System Usability Scale (SUS). The SUS includes 10 questions with a 5-point scale ranging from 'strongly disagree' to 'strongly agree'. These questions result in a 0 to 100 score on satisfaction of the novel technique, from highly unusable to highly usable [14]. A SUS score $\geq 70$ is considered good with a high chance of acceptance of the technology by the users [15]. For each odd-numbered question: subtract 1 from the score. For each of the even-numbered questions: subtract their value from 5. Multiply the sum of the scores by 2.5 to obtain the overall score. In our questionnaires, the total score was 75 . Second, questions to compare the conventional setting to the innovative navigation setting were included. With these questions, the effectiveness (survival, complications and resection margins), efficiency (extra time, tumor localization) and decisiveness during surgery were reviewed. A 5-point Likert scale was used in which a score above 3 was in favor of the innovative navigation setting. Additionally, the required time to set up the system - including positioning of the patient trackers and acquiring the intraoperative $\mathrm{CBCT}$ imaging - was reported. 


\section{Statistical analysis}

All analyses were performed in SPSS v $25.0^{\circ}$ (IBM Corporation; Armonk, NY, USA). Patient characteristics were presented as number (percentage) or median (range). Categorical variables were compared with a Fisher's exact test and continuous variables with the Mann-Whitney $U$ test. The $95 \%$ confidence intervals of percentages were calculated using the Clopper Pearson method. A logistic regression model was used to evaluate whether the association of the navigation system with surgical resection margins remained significant after adjusting for neoadjuvant therapy. A two-sided $p$-value of 0.05 or less was considered statistically significant.

\section{Results}

Between February 2016 and September 2019, 64 patients were included in the prospective abdominal navigation study. From these patients, 33 patients (10 (30.2\%) female; median age: 60 years (IQR: 55.0 - 68.50)) met the inclusion criteria and were included in the current analysis. Fourteen patients with locally advanced primary cancer and 19 patients with recurrent rectal cancer (Table 1). In all 33 patients, the image-guided navigation system was used without technical failures. In the OR, the required time to set up the navigation system was on average 16 minutes (SD: 6.0).

Between February 2016 and September 2019, a total of 85 patients with locally advanced primary and recurrent rectal cancer were operated in our hospital. In 52 patients, no navigation was performed mainly because surgeons considered radical resection less challenging or in some patients because of logistic reasons (no availability of the navigation system or hybrid OR).

\section{Surgical resection margins}

With image-guided navigation, a radical resection (R0) was accomplished in 13/14 patients (92.9\%) (95\% Cl: 66.1 - 99.8) with primary locally advanced rectal cancer and in $15 / 19$ patients (78.9\%) (95\% Cl: 54.4 - 94.0) with locally recurrent rectal cancer.

\section{Safety}

No navigation-related complications occurred before or during rectal surgery in the locally advanced primary or recurrent rectal cancer cohorts. In the locally advanced primary cohort, also no intraoperative complications occurred. In the recurrent rectal cancer cohort, intraoperative complications occurred in 4 patients: 1 patient with ureter injury and 3 patients with intra-abdominal bleeding during surgery. None of these complications could be attributed to using the image-guided navigation system. 
Table 1. Baseline characteristics

\begin{tabular}{|c|c|c|c|}
\hline & & & \\
\hline & Navigation group & Control group & P-value \\
\hline Locally advanced primary rectal cancer & $n=14$ & $n=101$ & \\
\hline Sex & & & 0.351 \\
\hline Male & $12(85.7)$ & $73(72.3)$ & \\
\hline Female & $2(14.3)$ & $28(27.7)$ & \\
\hline Age at start treatment (median, years) & $58.0(35-71)$ & $61.0(25-82)$ & 0.486 \\
\hline Clinical tumor and nodal stage & & & 0.562 \\
\hline T3N0-2 MRF+ & $7(50)$ & $62(61.4)$ & \\
\hline T4N0-2 MRF+ & $7(50)$ & $39(38.6)$ & \\
\hline Distant metastases present & $3(21.4)$ & $24(23.8)$ & 1.00 \\
\hline Primary tumors location from anorectal verge & & & 0.461 \\
\hline Low $(0-5 \mathrm{~cm})$ & $10(71.4)$ & $52(51.5)$ & \\
\hline Middle $(5-10 \mathrm{~cm})$ & $3(21.4)$ & $31(30.7)$ & \\
\hline High $(10-15 \mathrm{~cm})$ & $1(7.1)$ & $18(17.8)$ & \\
\hline Neoadjuvant treatment & & & 0.130 \\
\hline None & $0(0)$ & $1(1.0)$ & \\
\hline Short course radiotherapy ( 5 x 5 Gy) & $0(0)$ & $3(3.0)$ & \\
\hline Chemoradiation & $8(57.2)$ & $80(79.2)$ & \\
\hline $5 \times 5$ Gy + chemotherapy & $5(35.7)$ & $16(15.8)$ & \\
\hline Chemoradiation + chemotherapy & $1(7.1)$ & $1(1.0)$ & \\
\hline Type of surgery & & & 0.103 \\
\hline Open APR & $2(14.3)$ & $38(37.6)$ & \\
\hline Lap. APR & $0(0)$ & $3(3.0)$ & \\
\hline Open LAR & $12(78.6)$ & $35(34.7)$ & \\
\hline Lap. LAR & $1(7.1)$ & $10(9.9)$ & \\
\hline Exenteration & $0(0)$ & $15(14.9)$ & \\
\hline Recurrent rectal cancer & $n=9$ & $n=41$ & \\
\hline Sex & & & 0.788 \\
\hline Male & $11(57.9)$ & $22(53.7)$ & \\
\hline Female & $8(42.1)$ & $19(46.3)$ & \\
\hline Age at start treatment (median, years) & $61.5(52-78)$ & $67.0(41-82)$ & 0.079 \\
\hline Tumor location & & & 0.560 \\
\hline Pelvic wall / presacral & $14(73.7)$ & $26(63.9)$ & \\
\hline Staple line recurrence & $5(26.3)$ & $15(36.6)$ & \\
\hline Neoadjuvant treatment & & & 0.061 \\
\hline None & $0(0)$ & $5(12.2)$ & \\
\hline Short course radiotherapy ( 5 x 5 Gy) & $0(0)$ & $2(4.9)$ & \\
\hline Chemotherapy & $1(5.3)$ & $2(4.9)$ & \\
\hline Chemoradiation & $11(57.9)$ & $29(70.7)$ & \\
\hline Chemoradiation + chemotherapy & $7(36.8)$ & $3(7.3)$ & \\
\hline Type of surgery & & & 0.100 \\
\hline Open APR & $3(15.8)$ & $19(46.3)$ & \\
\hline Open LAR & $4(21.1)$ & $6(14.6)$ & \\
\hline Exenteration & $8(42.1)$ & $14(34.2)$ & \\
\hline Local resection & $4(20.0)$ & $2(4.9)$ & \\
\hline
\end{tabular}

\section{Comparison with historical controls}

From January 2009 to December 2015, 142 patients (47 (33.1\% females; median age: 62 years (IQR: 55.0 - 72.0)) with similar inclusion criteria received rectal surgery without image-guided navigation. In total, 101 patients with locally advanced primary rectal 


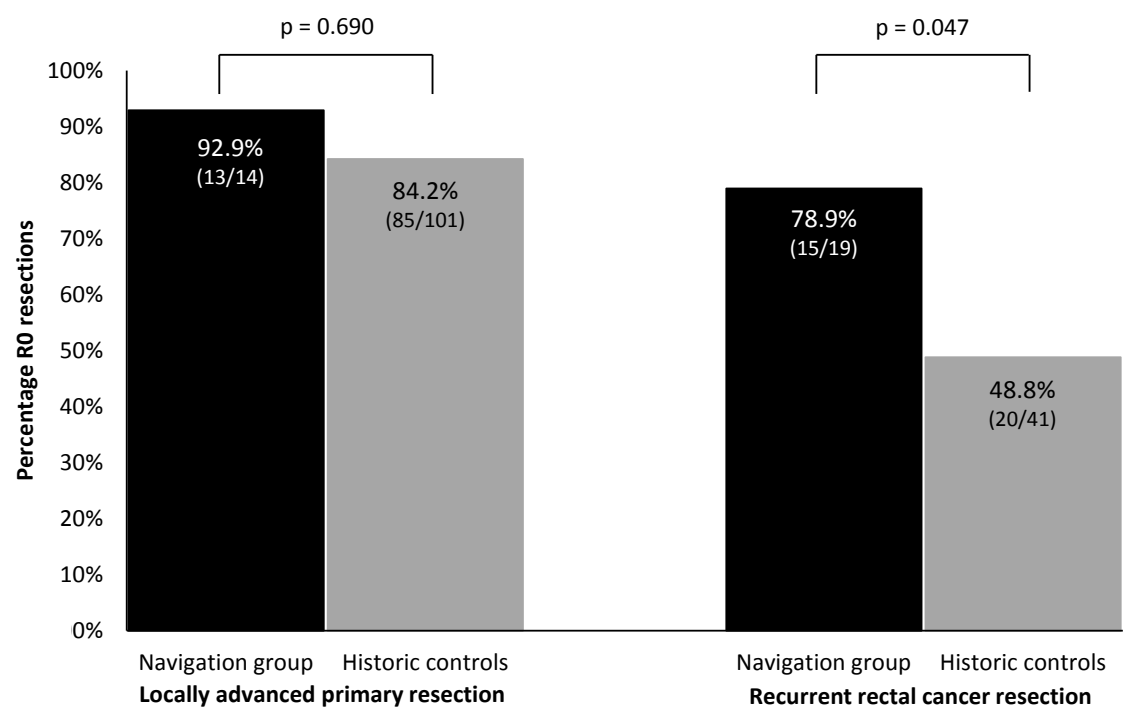

Figure 2. Comparison of complete resection rates between the navigation and historic cohort for patients with locally advanced primary and recurrent rectal cancer resection

cancer and 41 patients with recurrent rectal cancer were included in the control cohort (Table 1).

After primary tumor resection for locally advanced rectal cancer, a complete resection (R0) was accomplished in 85/101 patients (84.2\%) (95\% Cl: 75.6 - 90.7). After resection of recurrent rectal cancer, an $\mathrm{R} 0$ resection was achieved in 20/41 patients (48.8\%) (95\% $\mathrm{Cl}: 32.9$ - 64.9). Patients in the navigation cohort showed significantly lower positive margins rates after recurrent rectal cancer surgery than patients in the historical cohort ( $p=0.047$ ). For the locally advanced primary tumor resections, the difference was not significant ( $p=0.690$ ) (Figure 2). In the historical cohort, intraoperative complications were observed in five patients after primary tumor resection: ureter injury $(n=2)$, intra-abdominal bleeding $(n=2)$ and spleen injury $(n=1)$. After recurrent rectal cancer resection, intraoperative complications occurred in six patients: abdominal bleeding $(n=2)$ and ureter injury $(n=4)$. There were no significant differences in intraoperative complications between the navigation group and historic controls.

When comparing the prospective cohort to the historical control cohort, no significant difference was observed (Table 1). Despite this, we analyzed the potential influence of the less balanced characteristic, neoadjuvant chemotherapy. After controlling for neoadjuvant therapy in a logistic regression model for patients with recurrent rectal cancer, the use of image-guided navigation was significantly associated with complete resection margins (OR: 4.10; 95\% Cl 1.07 - 15.78; $\mathrm{p}=0.040$ ). After locally advanced primary tumor resection, the use of image-guided navigation was not significant 
association with complete resection margins after controlling for neoadjuvant therapy (OR: 2.46; 95\% Cl $0.29-20.72 ; \mathrm{p}=0.41$ ).

Because of a possible selection bias due to the restrictive inclusion by the surgeons, no analysis was performed on the patient cohort that did not undergo navigation in the period between February 2016 and September 2019. Baseline characteristics between patients with and without navigation differed considerably with regard to e.g cTNM and ypTNM (all in favor of the non-navigation group) which would make any comparison invalid.

\section{Usability of the system}

Nine individual surgeons performed surgery with the navigation system and completed 21 questionnaires in total. Eighty-six percent of all procedures were classified by the surgeons as 'complex'. In all complex cases, surgeons stated that the navigation system simplified the procedure. The navigation technique was scored with a median SUS score of 75.0 (range $42-87$ ) which indicates a high level of usability with a great chance of acceptance of the technique (Figure 3). In $84 \%$ of the questionnaires, surgeons reported that they wanted to use the technology during future procedures. The questions comparing the conventional setting to the innovative navigation setting showed a mean score of 3.9 (SD = 0.4), indicating surgeons preferred the navigation technique over the conventional setting. Especially questions about tumor localization,

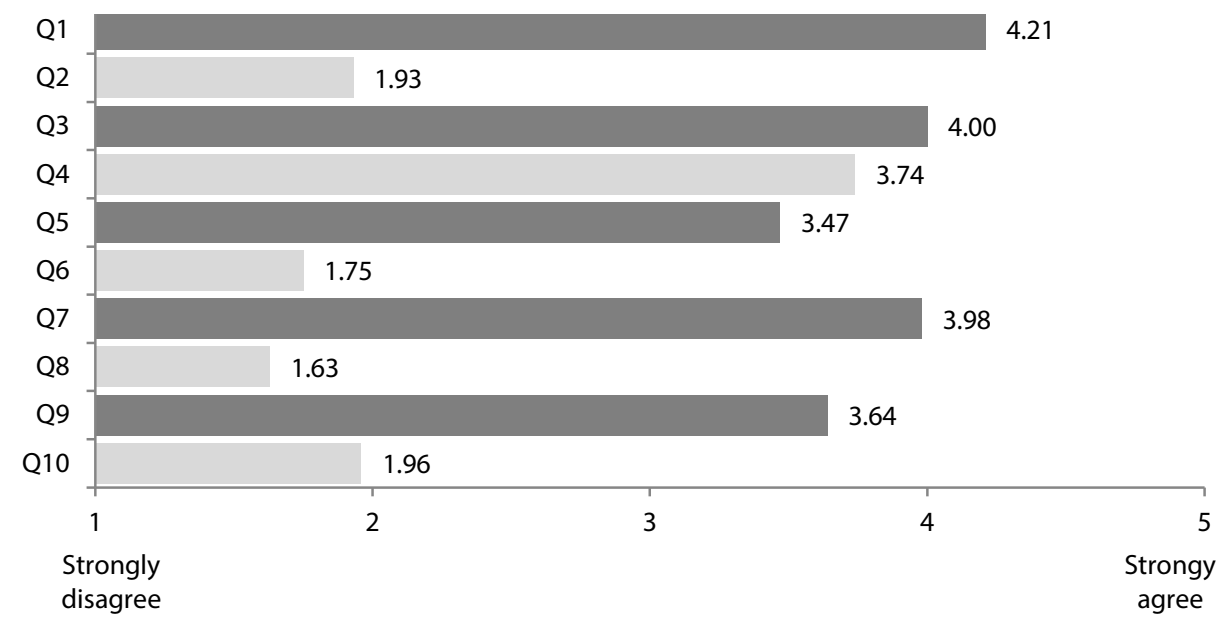

Figure 3. Mean item scores of the System Usability Scale. Questions in dark gray should be scored positively and questions in light gray should be scored negatively for a high usability score.

Q1: I think that I would like to use the system frequently. Q2: I found the system unnecessarily complex. Q3: I thought the system was easy to use. Q4: I think that I would need the support of a technical person to be able to use it. Q5: I found the various functions in the system were well integrated. Q6: I thought there was too much inconsistency in the system. Q7: I would imagine that most people would learn to use the system very quickly. Q8: I found the system very cumbersome to use. Q9: I felt very confident using the system. Q10: I need to learn a lot before I could get going with the system 


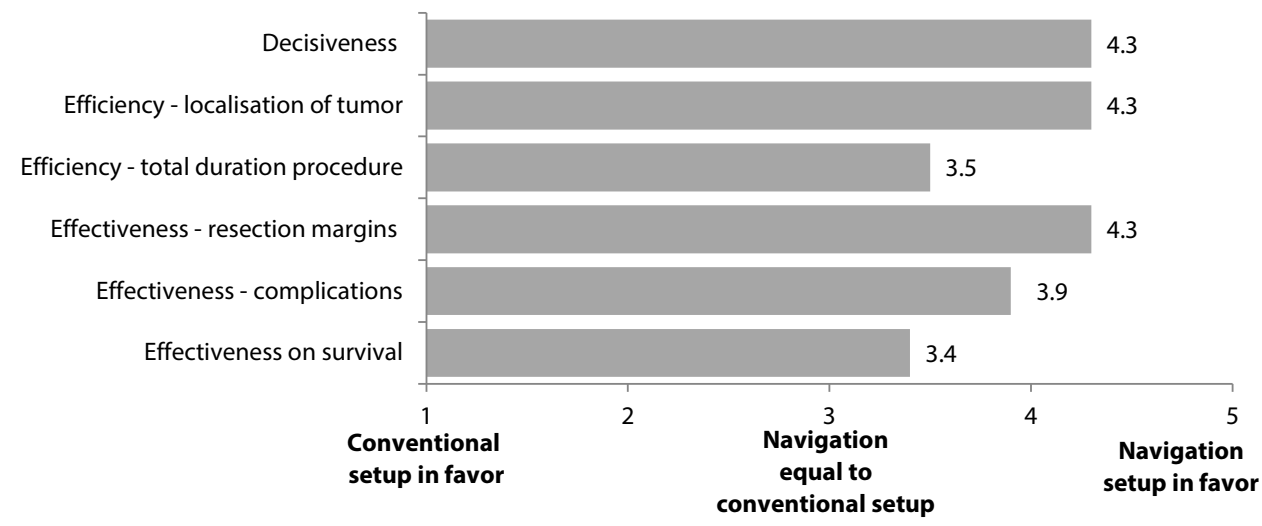

Figure 4. Results of questionnaires comparing the innovative navigation setting to the conventional setting without navigation. Numbers are presented as the mean scores.

securing resection margins and decisiveness scored high in favor of the innovative navigation setting (Figure 4).

\section{Discussion}

Patients with locally advanced primary and recurrent rectal cancer who underwent an image-guided surgical procedure showed high complete resection rates. The system demonstrated to be safe and no navigation-related complications were observed. In patients with recurrent rectal cancer, image-guided surgery was associated with increased resection margin rates, even after adjustment for neoadjuvant therapy. Surgeons rated the navigation system favorable over the conventional setting and stated that the technology improved decisiveness and simplified complex procedures.

Image-guided navigation in pelvic surgery has been described previously. Two individual groups investigated the feasibility of navigation in pilot studies for TransAnal Minimal Invasive Surgery - Total Mesorectal Excision (TAMIS-TME) [16-19]. Both concluded that navigation was feasible and safe, with satisfactory accuracy for clinical use. Although promising results were shown, only small patient cohorts of respectively 1 to 3 patients were evaluated. The first study in which image-guided navigation was tested in a large group, was performed by our group [13]. Outcomes of this pilot study were encouraging, showing the navigation system was safe, feasible and accurate (mean target registration error: $4.0 \mathrm{~mm}$ ) during pelvic surgery. However, the study included a wide variety of pelvic tumors and mainly focused on the feasibility and accuracy of the navigation system. The current article aimed at evaluating clinical outcomes of surgical navigation specifically in rectal cancer patients. This is the first 
study comparing resection margin rates of patients receiving rectal surgery with or without image-guided navigation.

In our study, a significant difference was found in surgical resection margins between the navigation and historical cohort in patients with locally recurrent rectal cancer. The positive resection margin rate in the historic control cohort was in line with other studies, varying between $38 \%$ and $62 \%[9,10,20]$. With the navigation, the percentage of positive resection margins was $21 \%$. In patients with locally advanced primary rectal cancer, no significant difference was found between the navigation and the historical cohort. This can mainly be explained by the generally much lower positive resection margin rates in primary rectal cancer surgery and by the small sample size of the navigation cohort. Literature on positive resection margins in rectal cancer often considers all different stages of rectal cancer. Data on the positive resection margin rate for locally advanced primary rectal cancer in the literature are scares, and numbers vary between the $9 \%-34 \%[1,9,10,21]$. The study of Rickles et al. included 8044 patients with cT3 rectal tumors and 773 patients with cT4 rectal tumors and showed tumor positive resection margins in $17.0 \%$ and $35.2 \%$ of the patients, respectively [1]. The results of the historical cohort in the current study, with $15.8 \%$ positive resection margins, are in line with these results. The $7.1 \%$ positive resection margins in the navigation cohort, although not statistically different from the historical cohort, compares favorably with this.

Integration of new surgical techniques requires evaluation of safety and usability. In this study, no navigation-related complications were observed, which is consistent with previous results [13]. The usability of the system was scored with a SUS score of 75 and surgeons preferred the navigation setting over the conventional setting in all questions. Moreover, the additional time for setting up the navigation system in our study was limited compared to other navigation studies, in which setting up required 25 to 47 minutes $[17,19]$. All these results suggest that our navigation system is safe and intuitive with a high chance of clinical acceptance outside the research setting.

Although promising results have been shown, some limitations must be acknowledged. This study had a non-randomized design, comparing a prospective cohort with a historical cohort, with its inherent methodological shortcomings. However, the historical control group we selected was well balanced with the navigation cohort with no differences in baseline characteristics. Nonetheless, there could be relevant differences in the characteristics of the two groups despite the fact that none of them were statistically significant. Therefore, even though the analysis plan did not specify any adjusted analysis, it was decided to adjust for neoadjuvant therapy. Second, this 
was a single-center study with small sample sizes in the navigation groups. Third, a dedicated clinical implementation team giving technical support was highly involved and it remains unclear if the system would have the same results when transferred to other hospitals. Last, the resection margin rates could have been influenced by the performance of the individual surgeons and the resulting quality of the total mesorectal excision specimen. Due to the retrospective set up of the historical cohort and the small sample size, these variables could not be taken into account. Nevertheless, this influence may be limited since in our institution we normally perform these kinds of procedures by a combined team of two experienced surgeons in rectal cancer, and results of the mesorectal resection specimen are discussed on a weekly basis in a multidisciplinary meeting.

In conclusion, image-guided surgery showed to be safe and effective, and was associated with an increase in radical resection margin rates in advanced rectal cancer surgery. This study is the first to compare resection margin rates in rectal cancer patients receiving surgery with or without image-guided navigation. Participating surgeons were excited about the improved decisiveness regarding resection margins and tumor localization. 


\section{References}

1. Rickles, A.S., D.W. Dietz, G.J. Chang, et al., High Rate of Positive Circumferential Resection Margins Following Rectal Cancer Surgery: A Call to Action. Ann Surg, 2015. 262(6): p. 891-8.

2. Bonjer, H.J., C.L. Deijen, G.A. Abis, et al., A Randomized Trial of Laparoscopic versus Open Surgery for Rectal Cancer. N Engl J Med, 2015. 372(14): p. 1324-1332.

3. Park, J.S., J.W. Huh, Y.A. Park, et al., A circumferential resection margin of $1 \mathrm{~mm}$ is a negative prognostic factor in rectal cancer patients with and without neoadjuvant chemoradiotherapy. Dis Colon Rectum, 2014. 57(8): p. 933-40.

4. Nagtegaal, I.D. and P. Quirke, What is the role for the circumferential margin in the modern treatment of rectal cancer? J Clin Oncol, 2008. 26(2): p. 303-12.

5. Heriot, A.G., P.P. Tekkis, A. Darzi, et al., Surgery for local recurrence of rectal cancer. Colorectal Dis, 2006. 8(9): p. 733-47.

6. Vermaas, M., F.T. Ferenschild, C. Verhoef, et al., Total pelvic exenteration for primary locally advanced and locally recurrent rectal cancer. Eur J Surg Oncol, 2007. 33(4): p. 452-8.

7. Harris, C.A., M.J. Solomon, A.G. Heriot, et al., The outcomes and patterns of treatment failure after surgery for locally recurrent rectal cancer. 2016. 264(2): p. 323-329.

8. Bhangu, A., S.M. Ali, A. Darzi, et al., Meta-analysis of survival based on resection margin status following surgery for recurrent rectal cancer. Colorectal Dis, 2012. 14(12): p. 1457-66.

9. Bhangu, A., S.M. Ali, G. Brown, et al., Indications and outcome of pelvic exenteration for locally advanced primary and recurrent rectal cancer. Ann Surg, 2014. 259(2): p. 315-22.

10. Nielsen, M.B., P.C. Rasmussen, J.C. Lindegaard, et al., A 10-year experience of total pelvic exenteration for primary advanced and locally recurrent rectal cancer based on a prospective database. Colorectal Dis, 2012. 14(9): p. 1076-83.

11. Hahnloser, D., H. Nelson, L.L. Gunderson, et al., Curative potential of multimodality therapy for locally recurrent rectal cancer. Ann Surg, 2003. 237(4): p. 502-8.

12. Senft, C., A. Bink, K. Franz, et al., Intraoperative MRI guidance and extent of resection in glioma surgery: a randomised, controlled trial. The Lancet Oncology, 2011. 12(11): p. 997-1003.

13. Nijkamp, J., K.F.D. Kuhlmann, O. Ivashchenko, et al., Prospective study on image-guided navigation surgery for pelvic malignancies. J Surg Oncol, 2019. 119(4): p. 510-517.

14. Brooke, J., SUS-A quick and dirty usability scale. 1996. 189(194): p. 4-7.

15. Bangor, A., P.T. Kortum and J.T. Miller, An Empirical Evaluation of the System Usability Scale. International Journal of Human-Computer Interaction, 2008. 24(6): p. 574-594.

16. Atallah, S., S.W. Larach, J.R.J.M.I.T. Monson, et al., Stereotactic navigation for TAMIS-TME. 2016. 25(5): p. 271-277.

17. Atallah, S., B. Martin-Perez and S.J.T.i.c. Larach, Image-guided real-time navigation for transanal total mesorectal excision: a pilot study. Techniques in Coloproctology, 2015. 19(11): p. 679-684.

18. Atallah, S., G. Nassif and S.J.S.E. Larach, Stereotactic navigation for TAMIS-TME: opening the gateway to frameless, image-guided abdominal and pelvic surgery. Surgical Endoscopy 2015. 29(1): p. 207-211.

19. Kwak, J.M., L. Romagnolo, A. Wijsmuller, et al., Stereotactic Pelvic Navigation With Augmented Reality for Transanal Total Mesorectal Excision. Dis Colon Rectum, 2019. 62(1): p. 123-129.

20. Heriot, A.G., C.M. Byrne, P. Lee, et al., Extended Radical Resection: The Choice for Locally Recurrent Rectal Cancer. 2008. 51(3): p. 284-291.

21. Yang TX, Morris DL, Chua TC. Pelvic Exenteration for Rectal Cancer: A Systematic Review. Dis Colon Rectum. 2013;56(4):519-531. 



\section{Chapter 7}

\section{Accurate surgical navigation with real-time tumor tracking \\ in cancer surgery}

Esther N.D. Kok

Roeland Eppenga Koert F.D. Kuhlmann

Harald C. Groen

Ruben van Veen

Jolanda M. van Dieren Thomas R. de Wijkerslooth Monique van Leerdam Doenja M.J. Lambregts Wouter J. Heerink

Nikie J. Hoetjes Oleksandra Ivashchenko Geerard L. Beets Arend G.J. Aalbers Jasper Nijkamp Theo J.M. Ruers

npj Precision Oncology (2020) 


\section{Abstract}

In the past decades, image-guided surgery has evolved rapidly. In procedures with a relatively fixed target area, like neurosurgery and orthopedics, this has led to improved patient outcomes. In cancer surgery, intra-operative guidance could be of great benefit to secure radical resection margins since residual disease is associated with local recurrence and poor survival. However, most tumor lesions are mobile with a constantly changing position. Here, we present an innovative technique for real-time tumor tracking in cancer surgery. In this study, we evaluated the feasibility of real-time tumor tracking during rectal cancer surgery. The application of real-time tumor tracking using an intraoperative navigation system is feasible and safe with a high median target registration accuracy of $3 \mathrm{~mm}$. This technique allows oncological surgeons to obtain real-time accurate information on tumor location, as well as critical anatomical information. This study demonstrates that real-time tumor tracking is feasible and could potentially decrease positive resection margins and improve patient outcome. 


\section{Introduction}

In the past two decades, image-guided surgery has evolved rapidly. The technique enables real-time visualization of surgical instrumentation with respect to intraoperative anatomy based on preoperative imaging. A prerequisite of the current surgical navigation systems is a fixed target area since registration of the pre-operative images with the intra-operative anatomy is based on this rigid, fixed position.

In view of this condition, the technique was initially developed in neurosurgery, in which the soft tissue component has a fixed position with respect to the bone. The main goal was to improve precise targeting of the tumor margins and improve the safety of the surgical procedures. Image-guided neurosurgery evolved from framebased stereotaxic to frameless navigation [1]. Nowadays, multiple studies showed that image-guided neurosurgery is feasible and improves the extent of resection of malignant brain tumors [2,3]. Additionally, the technique expanded to orthopedic and head and neck surgery where it showed to be of added value with improved accurate placement of surgical implants [4, 5].

Besides these applications, intra-operative guidance could be of great benefit to many other surgical oncology procedures not confined to a rigid surrounding. During such procedures, surgeons rely on palpation and visual inspection to distinguish tumorous from healthy tissue. This can be challenging and may result in incomplete removal of the tumor tissue, associated with local recurrence and poor overall survival [6-9]. To make navigation useful under such circumstances and improve surgical outcomes, the technique has to account for the changing position of the tumor by real-time tumor tracking during surgery.

In this study, we present a new technique that brings surgical navigation to the next stage of development and make it suitable for a wide range of applications in surgical oncology. To this end, we developed an innovative technology that allows for real-time tumor tracking during surgery. Here, we report on the feasibility and accuracy of this innovative technique in patients undergoing rectal surgery. In rectal cancer surgery, total mesorectal excision (TME) is the golden standard. TME can be challenging with limited visualization and surgical access due to the anatomical location of the tumor. Because of this, the amount of tumor positive resection margins still remains $10-15 \%$ $[10,11]$. Therefore, better intra-operative guidance would be of great benefit to secure radical resection margins.

The study was performed in 2 phases. In the first phase (feasibility phase), feasibility and safety of the study workflow were investigated, the possibility of real-time tumor tracking with the navigation system was assessed and possible improvements of 
the clinical workflow were identified. In the second phase (test phase), the ultimate accuracy of the real-time tracking was measured.

\section{Methods}

\section{Clinical trial design and patient inclusion}

This prospective feasibility study was conducted in The Netherlands Cancer Institute from November 2016 to August 2019. The study was approved by the institutional review board in August 2016 (NL57251.031.16/N16TRS). The study was conducted according to the principles of the Declaration of Helsinki and in accordance with the Medical Research Involving Human Subjects Act (WMO). Inclusion criteria were: patients with rectal cancer scheduled for open or laparoscopic low anterior resection (LAR) or abdominoperineal resection (APR), patient age $\geq 18$ years, tumor distance from anal verge $<10 \mathrm{~cm}$ (based on pre-operative imaging). Exclusion criteria were: contraindications for intravenous contrast administration (allergy or severely impaired kidney function), cardiac pacemaker and metal implants in the pelvic area causing artifacts on the preoperative imaging. All participating patients gave written informed consent. The study was registered at www.trialregister.nl (identifier: NL7666).

The study was performed in 2 phases. In the first phase (feasibility phase), 15 patients were included. The feasibility and safety of the study workflow were investigated, the possibility of real-time tumor tracking with the navigation system was assessed and possible improvements of the study protocol were identified. After optimization of the study protocol, the second phase (test phase) was initiated. In this test phase, 16 patients were included and the accuracy of the real-time tracking was determined.

\section{Navigation system}

An NDI Aurora V2 electromagnetic (EM) tracking system (Northern Digital Inc, Waterloo, Ontario, Canada) was used to link preoperative imaging data to the intraoperative patient setup. Patients were positioned on an operating table including a tabletop field generator (TTFG) with an oval EM field of $42 \times 60 \times 60 \mathrm{~cm}$. Patient trackers (Philips Traxtal/percunav, Philips, Best, The Netherlands) with EM sensors were used to determine the position of the patient during surgery and a tracking sensor was placed against the tumor to adjust for real-time tumor movements. Within a hybrid operating room, a cone-beam computed tomography (CBCT) scan (Philips Allura FD20 XperCT; Philips) was performed after sensor placement but before the start of surgery. Acquired images were registered to the pre-operative diagnostic planning CT-scan using the inhouse developed navigation software. During surgery, an EM-tracked surgical pointer (NDI) was available to validate the accuracy of the registration. 


\section{Workflow in feasibility phase}

A preoperative diagnostic multiphase contrast-enhanced abdominal CT scan (planning CT with early arterial and excretion phase) - acquired for the purpose of this study one day before surgery - and diagnostic magnetic resonance imaging (MRI) scans were used to create a patient-specific 3D model. The 3D model consisting of bones, arteries, veins, ureters, rectum, and tumor was created using semi-automated inhouse developed segmentation software. Tumor delineation was supervised by an experienced radiologist or study investigator with extensive experience in rectal cancer MRI. Resulting 3D models were evaluated by the operating surgeon before surgery. In the operation room (OR), 3 patients trackers (PercuNav Patient Tracker, Philips) were taped to the skin of the patient. One patient tracker was positioned at the level of the anterior superior iliac spine and the remaining two left and right of the spine in the lumbar curvature. Under anesthesia, a rectal examination was performed by the surgeon. With a proctoscope, a wired 6 degree of freedom EM-sensor (Flextube, Northern Digital Inc., Waterloo, Ontario, Canada) was fixated on the tumor using surgical glue. The patient was placed in surgical position and a CBCT image of the pelvic area including the patient trackers was acquired.

Because of the high mobility of the rectum, accurate registration of the intraoperative $\mathrm{CBCT}$ scan to the planning CT consisted of two steps: (I) rigid registration of the (semi) rigid parts of the model (i.e., bones and main vessels) and (II) deformable registration of the tumor. First, the bony anatomy on the intraoperative CBCT scan was linked to the 3D-model by rigid registration. Subsequently, maintaining the registration of the bones and semi-rigid anatomy unchanged, a separate deformable tumor registration was performed based on grey-values. The resulting navigation information consisted of the rigid anatomy of the pelvis plus a non-rigid representation of the tumor. The tumor sensor and pointer were automatically real-time tracked by the system and visible on a computer screen in the OR [24]. On this screen, the 3D model and 3 orthogonal views (axial, sagittal and coronal) of the obtained images were shown (Figure 1).

At the start of surgery, the actual position of the pointer was visually compared to the pointer position in the navigation system. In case of a visual inaccuracy of $\geq 2 \mathrm{~mm}$, the registration was adjusted accordingly. During further surgery, visual evaluation of the registration accuracy was performed by placing the tip of the pointer at anatomic landmarks like the sacral promontory, ureters or the common iliac artery bifurcation. If necessary, manual correction was performed on both the rigid anatomy as well as the tumor. 


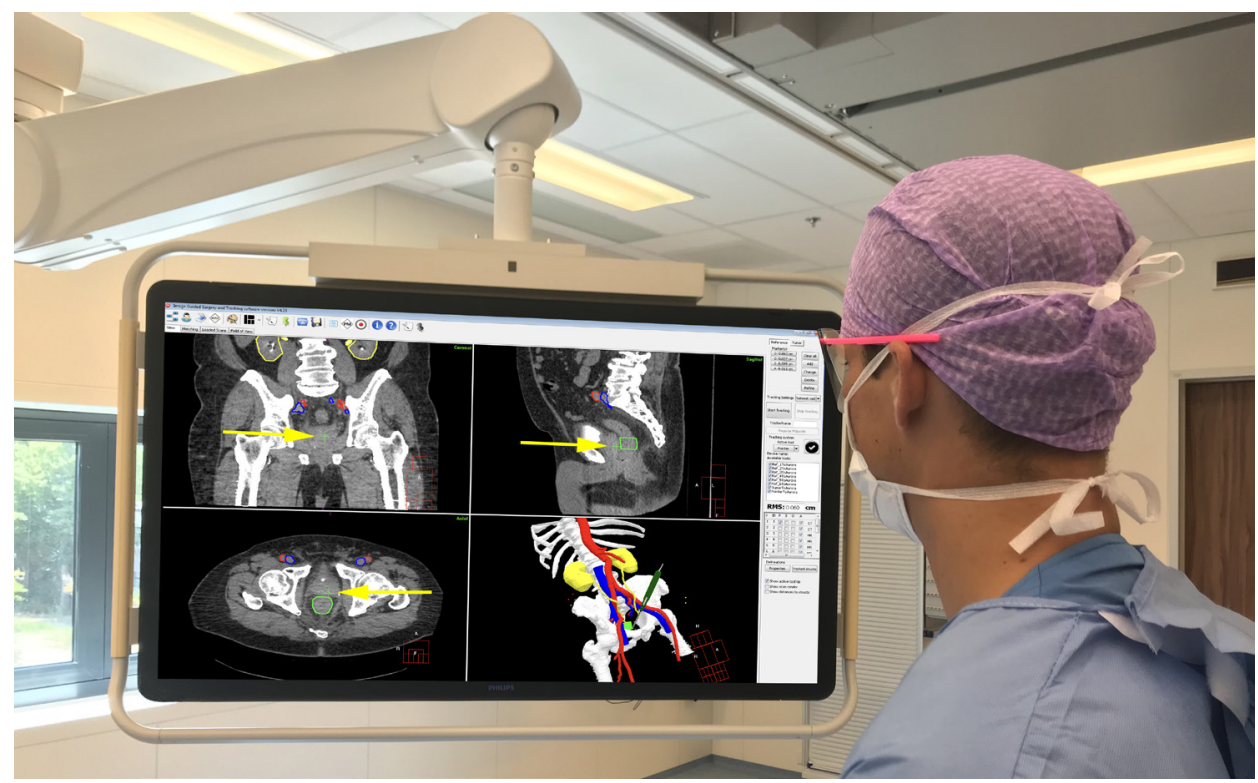

Figure 1. Surgical navigation user interface during surgery showing the planning CT with segmentations (top-left corner: coronal view; top-right corner: sagittal view; lower-left corner: axial view) and 3D model (lower-right corner). Visible segmentations: bones (white), arteries (red), veins (blue), ureters/kidneys (yellow), and tumor (green). The visible slice of the CT scans is based on the location of the tip of the surgical pointer which is highlighted with yellow arrows.

\section{Workflow in test phase}

For the test phase, the clinical workflow was changed with regard to tumor sensor placement and registration of the tumor. To secure protection for breakage and guarantee a more stable tumor sensor position, the sensor was inserted in a round silicone surgical wound drain $(3.3 \mathrm{~mm})$ before placement into the rectum. After positioning the tip of the sensor against the rectal tumor, the silicone wound drain was fixated to the skin in the perineal area with 2 sutures.

In the feasibility phase, tumor registration was challenging due to the low soft-tissue contrast on the $\mathrm{CBCT}$. In the test phase, to improve tumor registration, patients received fiducial markers close to the rectal tumor before the diagnostic CT scan, at least one week before surgery. These markers were used to facilitate tumor registration between the low soft-tissue contrast CBCT images and the high soft-tissue contrast of the planning CT. Fiducial placement in the rectum is often used for tumor localization during radiotherapy and has been reported as safe with excellent visibility on CT [25-27]. Patients received an enema before the procedure. Using flexible sigmoidoscopy in combination with endoscopic ultrasonography (EUS), an experienced gastroenterologist inserted 3 fiducials through the bowel wall in the mesorectal fat (one on the proximal and distal tumor border and one halfway the tumor). The locations of the fiducials were assumed 
to be rigid with respect to the tumor. Two types of fiducial markers were used: Gold Anchor GA150-20 0.28 mm x 20 mm unfolded length (Naslund Medical AB, Huddinge, Sweden) and Visicoil $0.5 \mathrm{~mm}$ x $5 \mathrm{~mm}$ (Core Oncology, Santa Barbara, California). The Gold Anchors were folded when inserted. Pre-operative fiducial placement by the gastroenterologist took 20 minutes on average. In the OR, after the bone to bone registration, registration of the tumor was performed based on the fiducial markers. This allowed for a more accurate intraoperative tumor registration and tracking.

\section{Accuracy measurements}

In the test phase, an axial plane was added to the 3D model to accentuate the proximal tumor border. During surgery, the surgeon used the navigation interface to place 2 titanium surgical clips on the surface of the rectum in the plane of the proximal tumor border as given by the 3D model. After the specimen was resected, it was taken to the pathology department and processed according to standard protocol [28]. The distance between the surgical clips and the proximal tumor border was macroscopically reported and verified with histopathology.

If the surgeon was not able to reach the proximal tumor border (as indicated by the navigation system) with the surgical instruments due to narrow pelvic space, surgical clips were placed as close as possible cranial of the axial plane. The difference between the distance of the clips relative to the proximal tumor border measured by the navigation system and the pathology measurements was used as accuracy value.

\section{Outcome parameters}

The main goal of the feasibility phase was to assess if real-time tracking of the rectal tumor using the in-house developed EM navigation system was feasible and safe. Feasibility was defined as successful completion of the whole investigational workflow resulting in continuous delivery of interpretable navigation data for rectal surgery. In addition, the amount of extra time needed to use the system during surgery was evaluated.

The main goal of the test phase was to evaluate the accuracy of the system, which was validated by the distance (in $\mathrm{mm}$ ) between the intraoperatively placed surgical clips, illustrating the proximal tumor border according to the 3D model, and the proximal tumor border as determined by pathology. In this phase, the amount of extra time was also reported. All analyses were performed in SPSS version $24.0^{\circledR}$ (IBM Corporation, Armonk, NY, USA). 


\section{Results}

\section{Feasibility phase}

Between November 2016 and March 2018, 15 patients with histological proven rectal cancer were included (Table 1). In all patients, a patient-specific 3D model was created based on preoperative imaging. The time to segment the preoperative images took around 2 hours. A schematic overview of the position of the tumor sensor that adjusted for real-time tumor movements is shown in Figure 2 and an example of the tracked tumor movement in Figure 3.

Table 1. Patient characteristics

\begin{tabular}{lcc}
\hline Characteristics & $\begin{array}{c}\text { Feasibility phase } \\
\mathbf{n = 1 5}\end{array}$ & $\begin{array}{c}\text { Test phase } \\
\mathbf{n}=\mathbf{1 6}\end{array}$ \\
\hline Sex & & $11(68.7)$ \\
$\quad$ Male & $10(66.7)$ & $5(31.3)$ \\
$\quad$ Female & $5(33.3)$ & $58(37-85)$ \\
Median age (years) (range) & $62(39-78)$ & $1(6.3)$ \\
Clinical tumor and nodal stage & & $2(12.5)$ \\
CT2N0 & $0(0.0)$ & $8(50.0)$ \\
CT2N+ & $2(13.3)$ & $5(31.3)$ \\
CT3N0 & $6(40.0)$ & $0(0.0)$ \\
cT3N+ & $5(33.3)$ & $0(0.0)$ \\
CT4N0 & $1(6.7)$ & $3.0(0-6)$ \\
CT4N+ & $1(6.7)$ & $1(6.3)$ \\
Median distance between anorectal verge and tumor (cm) & $3.0(0-9)$ & $4(25.0)$ \\
Neoadjuvant treatment & & $9(56.3)$ \\
Radiotherapy (5 x 5 Gy) & $3(20.0)$ & $1(6.3)$ \\
Radiotherapy (5 x 5 Gy) + chemotherapy & $5(33.3)$ & $1(6.3)$ \\
Chemoradiation (25 2 Gy + capecitabine) & $7(46.7)$ & $0(0)$ \\
Chemoradiation + chemotherapy & $0(0)$ & \\
Chemoradiation and contact radiotherapy & &
\end{tabular}

${ }^{1}$ Clinical tumor and nodal stage at diagnosis

In the feasibility phase, 11 patients (73.3\%) successfully completed the entire investigational workflow and received rectal surgery with the navigation technique. In 4 patients (26.7\%) the tumor sensor was damaged during placement and discontinued working. Therefore, the sensor was inserted in a silicone round surgical wound drain $(3.3 \mathrm{~mm})$ for protection before placement into the rectum in the subsequent patients (starting at patient 9). During the feasibility phase, tumor registration was challenging due to the low soft-tissue contrast on the CBCT. Therefore, patients (starting at patient 7) received fiducial markers close to the rectal tumor before the planning $C T$ scan, at least one week before surgery. In the $\mathrm{OR}$, registration of the tumor was performed based on the fiducial markers which allowed for a more accurate intraoperative tumor registration and tracking. Visual evaluation of the registration during surgery, when 


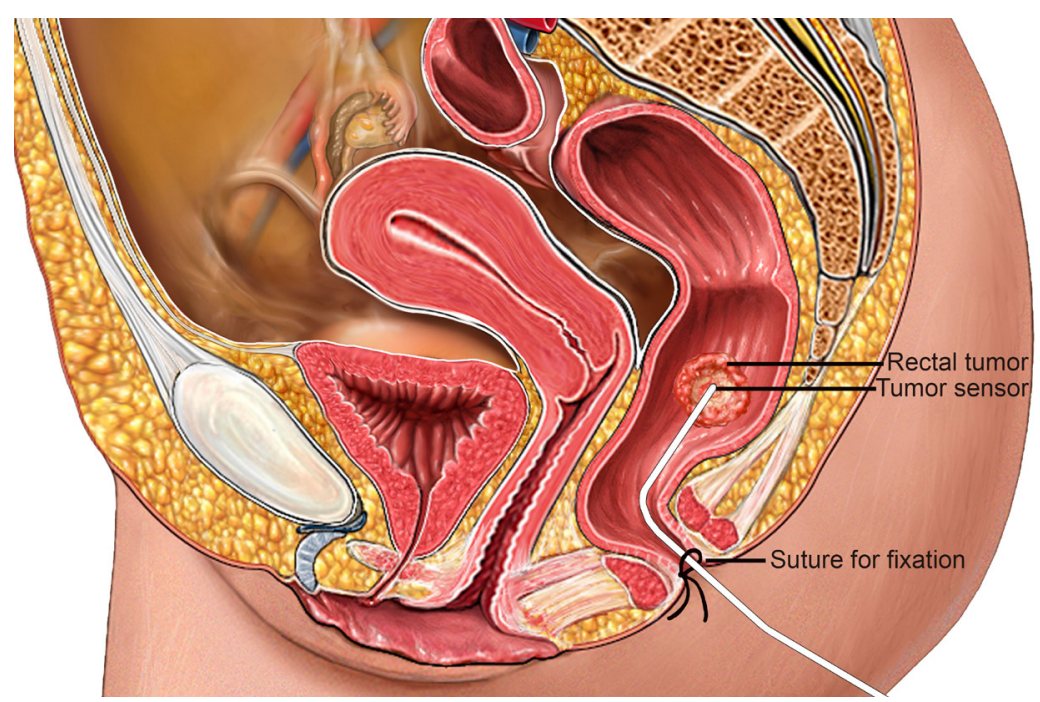

Figure 2. Schematic overview of in vivo tumor sensor with the tip positioned by the surgeon at the rectal tumor. The sensor is fixated with sutures in the perineal area. (Alamy Stock Photo (https://www.alamy. com/search.html?qt=Sagittal\%20View\%20of\%20a\%20Female\%20Pelvis\&imgt=0), used and edited with permission).

the patient was in Trendelenburg position, showed that manual correction of the system was necessary in 4 patients with an average of $3.8 \mathrm{~mm}$ in the left-right direction, $9.4 \mathrm{~mm}$ caudal-cranial direction and $5.5 \mathrm{~mm}$ in anterior-posterior direction. In one patient, the intratumoral fiducial was lost after placement. Registration was based on the remaining 2 fiducials in this patient. No navigation-related complications were observed. Treatment-related characteristics are shown in Table 2. Placement of the patient trackers, positioning the tumor sensor and obtaining the intraoperative $C B C T$ scan added on average 31 minutes (range: 21 - 46) to the total surgical procedure time.

\section{Test phase}

During the test phase, the definite protocol was evaluated in 16 patients (Table 1). Of these patients, 14 patients received rectal surgery with the navigation system. In two patients, navigation was not used during surgery. In the first patient, rectal surgery was canceled due to unexpected progression of metastatic disease. The second patient suffered from an intra-abdominal infection possibly related to the fiducial placement and underwent early resection without navigation. Treatment-related characteristics are shown in Table 2. During surgery with the patient in Trendelenburg position, adjustment of the registration was performed in 6 patients. The average correction was $2.3 \mathrm{~mm}$ in the left-right direction, $13.0 \mathrm{~mm}$ caudal-cranial direction and $3.8 \mathrm{~mm}$ in anterior-posterior direction. In one patient, the intratumoral fiducial was lost after 


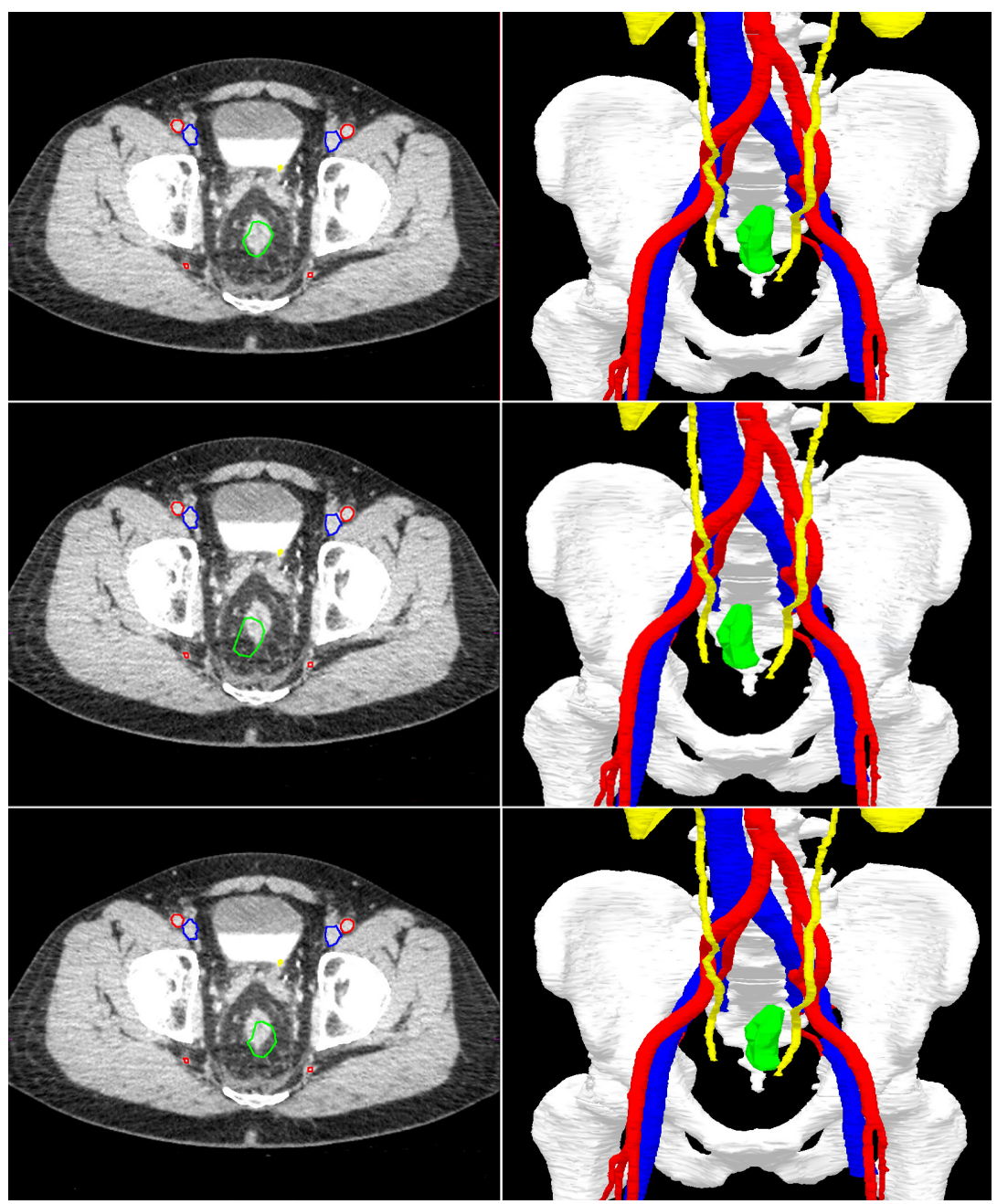

Figure 3. An example to illustrate the real-time tumor tracking by the navigation system. The top 2 images show the position of the rectal tumor in rest. In the lower 4 images, the rectum is moved to the right and left. Note that the green segmentation area follows the real-time tumor position in the navigation images on the right, while the CT scan on the left (still) shows the original position of the tumor. Visible segmentation: bones (white), arteries (red), veins blue), ureters/kidneys (yellow), tumor (green).

placement, resulting in a tumor registration based on the remaining 2 fiducials in this patient.

In the test phase, placement of the patient trackers, positioning of the tumor sensor and obtaining the intraoperative CBCT scan added on average 21 minutes (range: 15 - 30) to the total surgical procedure time. Real-time tracking of the rectal tumor was feasible in 13 of the 14 patients (92.9\%). In one patient, despite manual correction during the visual evaluation, no accurate registration could be achieved and the registration 
error remained $>2 \mathrm{~cm}$. Therefore, no interpretable navigation data could be collected. No navigation-related complications were observed during surgery.

Accuracy of the navigation set up was determined by correlation of the position of the proximal tumor margin, as determined during navigation and marked by surgical clips, with the position of the proximal tumor margin as determined on the pathology specimen. Accuracy measurements could be achieved in 11 patients (84.6\%) of the 13 patients with navigation data receiving rectal surgery. In one patient pathological correlation of tumor margins was not possible due to a complete pathological response (TON0) after neoadjuvant treatment and in one other patient because the surgical clips to mark the proximal tumor margin during navigation fell off the rectal resection specimen during surgical removal. The median absolute distance between the surgical clips and the proximal tumor border at histopathology was $3 \mathrm{~mm}$ (IQR: 2 - $10 \mathrm{~mm}$ ).

Table 2. Treatment characteristics

\begin{tabular}{lcc}
\hline Characteristics & $\begin{array}{c}\text { Feasibility phase } \\
\mathbf{n = 1 5}\end{array}$ & $\begin{array}{c}\text { Test phase } \\
\mathbf{n = 1 6}\end{array}$ \\
\hline Fiducial markers & $8(53.3)$ & $16(100.0)$ \\
Type of surgery & & $4(25.0)$ \\
$\quad$ Open APR & $4(26.7)$ & $4(25.0)$ \\
$\quad$ Lap. APR & $3(20.0)$ & $3(18.8)$ \\
$\quad$ Open LAR & $5(33.3)$ & $3(18.8)$ \\
$\quad$ Lap. LAR & $3(20.0)$ & $2(12.5)$ \\
$\quad$ No navigated surgery performed & $0(0)$ & $14(87.5)$ \\
Surgery with navigation & $11(73.3)$ & $0(0)$ \\
Technical navigation errors & & $1(6.3)$ \\
$\quad$ Tumor sensor-related & $4(26.7)$ & $1(6.3)$ \\
$\quad$ Registration-related & $0(0)$ & \\
Study-related severe adverse events ${ }^{1}$ & $0(0)$ & $0(0)$ \\
Intraoperative complications & & $1(6.3)$ \\
$\quad$ Navigation related & $0(0)$ & $21(15-30)$ \\
$\quad$ Non-navigation related & $0(0)$ & \\
Median extra time needed (min - max) & $31(21-46)$ & \\
\hline
\end{tabular}

'One patient suffered from an intra-abdominal infection possibly related to the fiducial placement

${ }^{2}$ One patient experienced iatrogenic injury of the ureter, not caused or related to the navigation system, and required reimplantation of the ureter in the bladder.

\section{Discussion}

Image-guided surgical navigation has become widespread in head and neck surgery, neurosurgery and orthopedics. In these fields, the technique is feasible due to the most rigid, bone-defined target area. To make surgical navigation a useful technique for cancer surgery in general, where tumor lesions often shift during surgery, real-time tumor tracking is necessary. In this article, we present a navigation system that fulfills this need and performs real-time tumor tracking during the course of surgery. The 
system was tested in rectal cancer surgery in both open and laparoscopic setting.

This prospective study shows that real-time tumor tracking with surgical navigation is feasible, safe and accurate. During the feasibility phase, improvements in the workflow were made to enhance the practicality, speed, and accuracy of the tumor registration. After optimization of the workflow, a high median accuracy of $3 \mathrm{~mm}$ was obtained for surgical navigation in the test phase of the study. No navigation-related complications were observed during surgery and the total extra time decreased with one-third towards the end of the study to 21 minutes. The accuracy obtained is clinically relevant and warrants further clinical implementation.

In the past years, some limited case series evaluated the feasibility of surgical navigation in pelvic surgery. The group of Atallah et al. published three articles, including 5 patients in total, on stereotactic navigation using an optical tracking system for TransAnal Minimal Invasive Surgery - Total Mesorectal Excision (TAMIS-TME) [1214]. In all three studies, they concluded that stereotactic navigation for TAMIS-TME was feasible and safe with an accuracy of around $4 \mathrm{~mm}$. However, the accuracy of the system did not assume tumor or patient movement during the procedure as the trackers were mounted on to the bedrail. Kwak et al. also performed navigation in pelvic surgery in 2 patients, stating that surgical navigation was feasible with acceptable accuracy of the target [15]. Although both groups showed encouraging results, no real-time tracking of the rectal tumor was performed and the rectal tumor was assumed to be fixed in the same position during the procedure.

The main challenge of the current study was to track tumor movement in realtime in every possible direction. This could be accomplished by introducing a 6 degree of freedom sensor close to the tumor lesion. The current results confirm earlier results from an ex vivo rectum study by Wagner et al [16]. They developed a realistic rectum phantom to test motion and deformation using a tracking sensor. Multiple models were created to translate sensor movement to rectum wall deformations. In the most complex model, 33 parameters were taken into account which resulted in a target registration error of $2.9 \pm 1.4 \mathrm{~mm}$. Our results are in accordance with the results obtained in these phantom experiments.

A limiting factor for the accuracy measurements in the current in vivo application for rectal cancer may be that all patients in the current study were operated after neoadjuvant radiotherapy with a long interval to surgery. This makes interpretation of the preoperative imaging more challenging. MRI is the most advanced staging technique in rectal cancer and routinely used for preoperative staging and restaging $[17,18]$. Unfortunately, interpretation of MRI is known to be less reliable when patients are pretreated with chemoradiation or radiotherapy. Multiple studies evaluated MRI for 
restaging and showed overall a low sensitivity, which is mainly caused by difficulties to differentiate areas of vital residual tumor within radiation-induced fibrosis [19, 20]. In this study, all segmentations of the tumor and other critical structures were validated by an experienced radiologist or a rectal cancer MRI expert and the operating surgeon. Despite this challenging task, a median navigation accuracy of $3 \mathrm{~mm}$ was established.

Besides the interpretation of the MRI, some other factors may have influenced the accuracy. For example, the obtained accuracy may be dependent on the positioning and registration of the fiducials. Fiducial placement was performed using a sigmoidoscopy in combination with endoscopic ultrasonography but in smaller limited-volume tumors, adequate positioning of the fiducials on the proximal and distal tumor was challenging. Furthermore, it remains uncertain if the position of the fiducials was indeed rigid with respect to the tumor. Another factor that might have influenced the accuracy is the pathological processing of the rectal specimen. The distance between the surgical clips and the proximal tumor border was measured manually by the pathologist and reproduction of the axial tumor plane was in some patients complex.

Manual correction of the registration was performed in 10 out of 29 patients. When corrections were applied, these were almost exclusively related to the caudal-cranial axis (9 out of 10). A possible explanation for the inaccuracy in this direction may be found in the way patients were tracked during surgery. Patient trackers were taped to the skin close to the bone to correct for patient movement with respect to the table and field generator. However, some movement of the skin and the patient trackers with respect to the pelvis may be inevitable, especially when the patient is moved to the Trendelenburg position during surgery. Obtaining the CBCT scan after the patient is positioned to Trendelenburg is unfortunately not possible since a security lock in the CBCT scanner prevents scanning when the bed is not positioned straight. However, this slight movement could easily be corrected for by visual correction of the registration after the patient was positioned in Trendelenburg.

During the study, adjustments were performed improving the practicality and the accuracy of the tumor registration. However, positioning and securing the tumor sensor against the rectal tumor remains inconvenient and adds additional time to the anesthesia. A solution may be found in the use of wireless EM tracked transponders [21-23]. These wireless transponders could be implanted near the tumor the day before surgery when the patient would be admitted to the hospital, and their position could be visualized by CT the same day. During the OR no additional imaging would be necessary, and any shift of tumor location could simply be tracked in real-time by tracking the responder position.

In conclusion, this study shows that real-time tumor tracking for cancer surgery 
is feasible and safe. The accuracy of the system is high, which justifies broader clinical implementation. Further studies will be initiated to improve the workflow and prove clinical benefit. 


\section{References}

1. Schulz, C., S. Waldeck, and U.M. Mauer, Intraoperative image guidance in neurosurgery: development, current indications, and future trends. Radiology research and practice, 2012. 2012: p. 197364-197364.

2. Senft, C., et al., Intraoperative MRI guidance and extent of resection in glioma surgery: a randomised, controlled trial. The Lancet Oncology, 2011. 12(11): p. 997-1003.

3. Claus, E.B., et al., Survival rates in patients with low-grade glioma after intraoperative magnetic resonance image guidance. Cancer, 2005. 103(6): p. 1227-33.

4. Tormenti, M.J., et al., Intraoperative computed tomography image-guided navigation for posterior thoracolumbar spinal instrumentation in spinal deformity surgery. Neurosurgical focus, 2010. 28(3): p. E11.

5. Aschendorff, A., et al., Radiologically assisted navigation in cochlear implantation for X-linked deafness malformation. Cochlear Implants Int, 2009. 10 Suppl 1: p. 14-8.

6. Shindoh, J., et al., Complete removal of the tumor-bearing portal territory decreases local tumor recurrence and improves disease-specific survival of patients with hepatocellular carcinoma. Journal of Hepatology, 2016. 64(3): p. 594-600.

7. Houssami, N., et al., The association of surgical margins and local recurrence in women with earlystage invasive breast cancer treated with breast-conserving therapy: a meta-analysis. Ann Surg Oncol, 2014. 21(3): p. 717-30.

8. El-Sherif, A., et al., Margin and Local Recurrence After Sublobar Resection of Non-Small Cell Lung Cancer. Annals of Surgical Oncology, 2007. 14(8): p. 2400-2405.

9. Adam, I.J., et al., Role of circumferential margin involvement in the local recurrence of rectal cancer. The Lancet, 1994. 344(8924): p. 707-711.

10. Rickles, A.S., et al., High Rate of Positive Circumferential Resection Margins Following Rectal Cancer Surgery: A Call to Action. Ann Surg, 2015. 262(6): p. 891-8.

11. Bonjer, H.J., et al., A Randomized Trial of Laparoscopic versus Open Surgery for Rectal Cancer. N Engl J Med, 2015. 372(14): p. 1324-1332.

12. Atallah, S., B. Martin-Perez, and S.J.T.i.c. Larach, Image-guided real-time navigation for transanal total mesorectal excision: a pilot study. Techniques in Coloproctology, 2015. 19(11): p. 679-684.

13. Atallah, S., G. Nassif, and S.J.S.E. Larach, Stereotactic navigation for TAMIS-TME: opening the gateway to frameless, image-guided abdominal and pelvic surgery. Surgical Endoscopy 2015. 29(1): p. 207211.

14. Atallah, S., et al., Robotic-assisted stereotactic real-time navigation: initial clinical experience and feasibility for rectal cancer surgery. Techniques in Coloproctology, 2019. 23(1): p. 53-63.

15. Kwak, J.M., et al., Stereotactic Pelvic Navigation With Augmented Reality for Transanal Total Mesorectal Excision. Dis Colon Rectum, 2019. 62(1): p. 123-129.

16. Wagner, M., et al., Electromagnetic organ tracking allows for real-time compensation of tissue shift in image-guided laparoscopic rectal surgery: results of a phantom study. Surg Endosc, 2016. 30(2): p. 495-503.

17. Jhaveri, K.S. and H. Hosseini-Nik, MRI of Rectal Cancer: An Overview and Update on Recent Advances. American Journal of Roentgenology, 2015. 205(1): p. W42-W55.

18. Li, X.-T., et al., Evaluating rectal tumor staging with magnetic resonance imaging, computed tomography, and endoluminal ultrasound: A meta-analysis. Medicine, 2016. 95(44): p. e5333-e5333.

19. van der Paardt, M.P., et al., Patients who undergo preoperative chemoradiotherapy for locally advanced rectal cancer restaged by using diagnostic MR imaging: a systematic review and metaanalysis. Radiology, 2013. 269(1): p. 101-12.

20. Kim, S.H., et al., Apparent diffusion coefficient for evaluating tumour response to neoadjuvant chemoradiation therapy for locally advanced rectal cancer. European Radiology, 2011. 21(5): p. 987995.

21. Franz, A.M., et al., Electromagnetic tracking in medicine-a review of technology, validation, and 
applications. IEEE Transactions on Medical Imaging, 2014. 33(8): p. 1702-1725.

22. Willoughby, T.R., et al., Target localization and real-time tracking using the Calypso 4D localization system in patients with localized prostate cancer. International Journal of Radiation Oncology* Biology* Physics, 2006. 65(2): p. 528-534.

23. Janssen, N., et al., Real-time wireless tumor tracking during breast conserving surgery. International Journal of Computer Assisted Radiology and Surgery 2018. 13(4): p. 531-539.

24. Nijkamp, J., et al., Comparing position and orientation accuracy of different electromagnetic sensors for tracking during interventions. Int J Comput Assist Radiol Surg, 2016. 11(8): p. 1487-98.

25. Moningi, S., et al., Analysis of fiducials implanted during EUS for patients with localized rectal cancer receiving high-dose rate endorectal brachytherapy. Gastrointestinal Endoscopy, 2015. 81(3): p. 765769.e1.

26. Vorwerk, H., et al., Gold markers for tumor localization and target volume delineation in radiotherapy for rectal cancer. Strahlentherapie und Onkologie, 2009. 185(2): p. 127-133.

27. van den Ende, R.P.J., et al., MRI visibility of gold fiducial markers for image-guided radiotherapy of rectal cancer. Radiotherapy and Oncology, 2019. 132: p. 93-99.

28. Campa-Thompson, M., et al., Pathologic processing of the total mesorectal excision. Clinics in colon and rectal surgery, 2015. 28(1): p. 43-52. 



\section{Chapter 8}

\section{General discussion}


In this thesis, the results of various treatment modalities for patients with colorectal cancer are presented. The focus of the first part was on new applications of existing treatment modalities.

In Chapter 2, the effectiveness of short-course radiotherapy, followed by systemic therapy and local treatment, also known as the M1 schedule, in (potentially) curable stage IV rectal cancer patients was investigated. In total, 169 patients from eight centers in The Netherlands received this treatment schedule in daily practice and were included in the study. The multimodal treatment showed adequate relief of symptoms in most patients with good survival rates in patients who completed the treatment schedule. The number of liver metastases $(\geq 4)$ at diagnosis was the only predictor of treatment completion and survival.

The optimal multimodality treatment schedule for patients with synchronous stage IV colorectal cancer remains controversial. The choice between neoadjuvant shortcourse radiotherapy plus chemotherapy or chemoradiation to treat the primary tumor is dependent on the local tumor stage, although randomized phase III study compared these treatment strategies in patients with locally advanced rectal tumors and found no difference in local efficacy [1]. Lower acute toxicity and improved overall survival was observed in patients treated with short-course radiotherapy and subsequent chemotherapy. The results in Chapter 2 are in line with these findings, showing that short-course radiotherapy followed by systemic chemotherapy (the M1 schedule) is an effective treatment with adequate (symptomatic and pathological) disease control of the primary tumor. According to the latest Dutch guidelines, short-course radiotherapy with delayed surgery should only be considered for locally advanced rectal tumors in case of high age or comorbidity [2]. Given the results mentioned above, short-course radiotherapy followed by systemic therapy should be considered more frequently as it shows to be as a good alternative in metastasized locally advanced rectal cancer patients.

An alternative treatment schedule that is often used in stage IV rectal cancer patients is known as the liver-first approach. Here, patients receive neoadjuvant chemotherapy followed by liver resection and subsequent (chemo-)radiotherapy before primary tumor resection. Comparing the results from a recently published Dutch article on the liver-first approach with our study shows similar results on local control and survival [3]. The main difference with the M1 schedule is that only patients with asymptomatic primary tumors are suitable for the liver first- 
approach [4, 5]. These findings emphasize that evaluating patients individually is crucial for optimal treatment of stage IV colorectal cancer patients.

The question remains, whether surgery will be necessary in all colorectal cancer patients in the future. Due to the improved neoadjuvant therapy schedules, approximately $20 \%$ of the rectal patients achieve a complete response $[6,7]$. In patients with a clinical complete response after neoadjuvant therapy, a Watch and Wait strategy can be considered [8-10]. A large multicenter study showed that the 2-year cumulative incidence of local regrowth in these patients was merely $25 \%$, of which $97 \%$ was located in the bowel wall [8]. The 5-year survival rate of the entire cohort was $85 \%$. The authors concluded that this new strategy avoids major resection in selected patients with little oncological risk. Despite these excellent results, only a small proportion of all colorectal patients are eligible for this strategy currently.

Although neoadjuvant therapies and surgical techniques improved largely over time, only a proportion of the patients with liver metastases are candidates for surgery. Research showed that systemic therapy may convert unresectable metastases into resectable lesions but in many stage IV patients, systemic treatment remains palliative because resection remains unfeasible $[11,12]$. Therefore, alternative local therapies are being investigated to still offer these patients with unresectable liver metastases a (potentially) curative treatment. In Chapter 3, the efficacy and safety of stereotactic body radiation therapy (SBRT) for unresectable liver metastases was evaluated. In total, 90 patients were included and divided into a low dose group of $\leq 100 \mathrm{~Gy}$ and a high dose group of $>100$ Gy near-minimum Biological Effective Doses $\left(\mathrm{BED}_{98 \%}\right)$. The two-year local control rate in the high dose group was excellent (85\%) and significantly better than in the low dose group (48\%). Furthermore, improved overall survival with similar toxicity rates were observed in patients who received high dose SBRT.

To determine whether SBRT is superior to other local treatments e.g. thermal ablation remains difficult. A Danish center initiated a phase III trial, comparing radiofrequency ablation (RFA) with SBRT, but was closed preliminary due to insufficient recruitment (NCT01233544). Unfortunately, there are no other randomized controlled trials comparing radiotherapy to other local therapies. The best available evidence comes from two retrospective studies, one comparing SBRT with RFA and the other comparing SBRT with microwave ablation (MWA) $[13,14]$. Both studies concluded that SBRT and RFA/MWA provided excellent and similar local control rates for small lesions. However, for larger lesions (for RFA $\geq 20 \mathrm{~mm}$ and for MWA $\geq 30 \mathrm{~mm}$ ) treatment with SBRT was 
associated with improved local control and therefore the preferable treatment.

Currently, SBRT is mainly used in patients with lesions not suitable for surgery or other local treatments. A recently published randomized phase II trial compared palliative systemic treatment to palliative systemic treatment plus stereotactic ablative radiotherapy (SABR) in patients with unresectable colorectal metastases [15]. They found a 13-month improvement in overall survival and a doubling of progression-free survival in the SABR group compared to the control group, with a small increase of early death in the SABR group. A prerequisite of this treatment strategy is that local treatment should be performed on all metastatic lesions. In our study, we also found that adequate local control is correlated with improved overall survival. These data strongly suggest that systemic treatment should be combined with local treatment of all tumor lesions to improve survival rates in patients with unresectable liver metastases. The survival benefit of adding local treatment to systemic treatment in patients with unresectable colorectal liver metastases has been described before with RFA or RFA in combination with resection as local therapy [16]. An advantage of thermal ablation over SBRT is that it can be used in patients with many hepatic lesions where radiation is limited to the amount of lesions. Despite that, SBRT is non-invasive and requires only brief outpatient clinic visits. Therefore, this should be preferred over invasive ablative treatment modalities in patients with a limited amount of lesions.

Positive results on SBRT for liver metastases have been published in both retrospective and phase I and II studies, with 2-year local control rates ranging from $66 \%-100 \%$ [17-20]. A disadvantage of the current SBRT setup is the lack of real-time anatomical information during treatment. A solution to mitigate this problem could be to integrate magnetic resonance imaging (MRI) in the radiation process. $M R I$ is capable of providing high-quality images with excellent soft-tissue contrast [21]. Therefore, an MR-Linac system allows real-time soft tissue imaging during radiotherapy and will enable adaptation of the treatment based on MR-imaging. Moreover, it makes fiducials that mark the tumor lesions, now implanted before treatment, superfluous. Our study showed that high dose SBRT provides excellent local control. Delivery of high dose SBRT using an MR-Linac seems promising to further improve these outcomes, and may even outpace surgical resection in certain cases, such as frail elderly patients.

In Chapter 4 we examined if neoadjuvant chemotherapy and/or targeted therapy could inhibit the growth of liver metastases induced by primary tumor resection. In this study, 47 colorectal cancer patients with synchronous unresectable liver metastases 
were included and randomized in either the control group or one of the three treatment groups. The control group received immediate resection of the primary tumor without any neoadjuvant treatment. Patients in the three treatment groups received neoadjuvant bevacizumab or capecitabine-oxaliplatin (CAPOX) or CAPOX with bevacizumab (CAPOX-B) with subsequent primary tumor resection, respectively. A significant increase in tumor volume and FDG uptake of the liver metastases on CT and PET-CT, respectively, was observed after primary tumor resection in the control group. In the treatment groups, no difference in tumor volume or FDG uptake was found. The study demonstrated that outgrowth of metastatic disease in colorectal cancer, as observed in the control group after resection of the primary tumor, can be inhibited by systemic therapy before primary tumor resection. Furthermore, CEA levels of patients treated with neoadjuvant CAPOX-B decreased significantly after primary tumor resection.

It remains unclear whether primary tumor resection is necessary in patients with unresectable colorectal liver metastases with an asymptomatic primary tumor. In the past, the majority of the patients received resection of the primary tumor but this number decreased over time [22]. With the advent of modern systemic therapies, primary chemotherapy has been widely accepted. A recently published systematic review and meta-analysis compared patients with the primary tumor still present to patients who received primary tumor resection [23]. In total, 159.991 patients with incurable metastatic colorectal disease were included. They concluded that resection of the primary tumor improved overall survival with a mean difference of 7.27 months $(p<0.0001)$ without a significant increase of morbidity. Although the presented review is in favor of primary tumor resection, some limitations must be acknowledged. No randomized controlled trials were included and the non-randomization of the included studies may have led to selection bias since patients who underwent primary tumor resection may have had a favorable performance status or a lower tumor burden.

Although our study focused on patients with unresectable colorectal liver metastases, the findings may well have a bearing in a wider range of colorectal cancer patients. Currently, patients with resectable localized colon cancer and tumor positive lymph nodes receive chemotherapy after the tumor is resected. But still, up to $25 \%$ of the patients develop metachronous distant metastases during follow up $[24,25]$. The timing of systemic therapy can, therefore, be questioned [26]. Treating patients with neoadjuvant systemic therapy instead of adjuvant therapy might lead to significant downsizing of the tumor lesion, as well as reduce the shedding of tumor cells and 
prevent outgrowth of the micrometastatic disease induced by surgical manipulation $[27,28]$. Some physicians are, however, concerned that patients with resectable colorectal cancer might show progression during the neoadjuvant treatment phase. The recently performed FOxTROT trial randomized 1052 patients with locally advanced colon carcinoma to neoadjuvant chemotherapy followed by surgery or surgery upfront followed by adjuvant chemotherapy [26, 29]. Preliminary results showed that neoadjuvant chemotherapy was feasible and well-tolerated without an increase in perioperative morbidity. Longer follow-up is, however, required to determine the longterm benefits on survival of neoadjuvant systemic treatment in these patients. So far, the results in the FOxTROT study are in line with our results. Overall, toxicity and surgical complications in our study were low and no emergency resections were required.

The second part of this thesis is focused on new surgical techniques for localized colorectal cancer. In the last decade, laparoscopic surgery has become common practice. Compared to open colorectal surgery, improved short-term surgical outcomes with similar recurrence and survival rates have been observed [30-32]. One of the drawbacks of laparoscopic surgery is the reduced tactile feedback, which makes tissue recognition more challenging. Especially in rectal cancer, where many patients are treated with neoadjuvant radiotherapy, significant rates (10-15\%) of positive resection margins are still observed $[33,34]$. To improve radical margins in colorectal cancer surgery in general, the use of hyperspectral imaging (HSI) as an additional imaging technique was evaluated in Chapter 5. In an ex vivo study, a sample of fat, healthy colorectal wall and tumor tissue was collected from 32 patients who received colorectal cancer surgery and imaged with two hyperspectral cameras. Two classification algorithms were used to discriminate the different tissue types. The overall accuracy to distinguish the three tissue types from each other was 0.88 (SD: 0.13). When the accuracy was determined per patient, an accuracy of 0.93 (SD: 0.12) was achieved. The results of this study showed the potential of HSI during colorectal surgery for fast tissue classification, which could increase the number of radical resections.

The only established 'real-time' technique for resection margin assessment in colorectal cancer surgery currently is intraoperative frozen section histology. During surgery, the surgeon resects a piece of tissue from a tumor-suspicious region in the abdomen. The resected tissue sample is frozen rapidly, after which it is cut, stained and interpreted by the pathologist [35]. The entire procedure takes between 20 and 30 minutes during which the patients remain under anesthesia. This technique has mainly been tested in rectal cancer for distal resection margin assessment to increase sphincter preservation 
rates. Especially in patients with lower rectal tumors treated with neoadjuvant chemoradiation, intraoperative frozen sections is recommended [36, 37]. In mid and lower rectal cancer surgery, frozen section has a sensitivity and specificity of $83 \%$ and $98 \%$, respectively [38]. Compared to frozen section analysis, HSI has a clear benefit. In frozen section analysis, there is a chance of sampling error resulting in false-negative outcomes. With HSI, larger areas can be imaged at once, making this technique more reliable. Moreover, in its ultimate form, HSI can be performed in real-time during surgery and doesn't require 30 minutes of waiting.

Where in some oncological surgical areas, multiple imaging techniques are being tested for intraoperative margin assessment, there are not many new techniques in colorectal cancer with this purpose. Some imaging techniques like multiphoton microscopy, Raman spectroscopy, and optical coherence tomography are being investigated for real-time tissue classification in colorectal cancer but these techniques are still in an experimental phase and will not be discussed in this thesis [39-41].

The ultimate goal of our study was to develop a real-time technique for tissue identification in laparoscopic colorectal surgery. The presented classification method already allows to apply HSI for real-time use but some technical changes should be made to enable the in vivo use of HSI. The used set-up included two push-broom cameras, where the samples were scanned by moving underneath the camera. This was a very controlled setting where the tissue slices were always at the same distance with respect to the camera and the light source. In an in vivo setting, this will not be possible. Therefore, a hyperspectral camera that operates in the visible and nearinfrared wavelengths ranges should be integrated in a laparoscope. Our research team is currently working on the development of such a laparoscope. This laparoscope should be tested on safety and effectiveness before in vivo measurements can be performed.

Hyperspectral imaging is not the only imaging technique that has been evaluated to improve surgical outcomes. In the past decades, image-guided navigation has been developed in fields like neurosurgery, orthopedics and head, and neck surgery. To investigate whether surgical navigation would also be possible in abdominal surgery, a navigation system was developed in-house and tested by our research group. This intraoperative technique showed to be feasible, safe and accurate in advanced pelvic surgery. In Chapter 6, the clinical benefit of this image-guided navigation system in advanced rectal cancer patients was evaluated. In this study, we compared resection margin rates of a prospective patient cohort operated with image-guided navigation 
to patients from a historical cohort, who received rectal cancer surgery without imageguided navigation. Both the prospective and historical cohort were subdivided into two groups: patients with locally advanced primary rectal cancer and patients with recurrent rectal cancer. Our results showed that patients with recurrent rectal cancer in the historical cohort had significantly higher positive resection margin rates compared to patients in the navigation cohort. In patients with locally advanced primary rectal tumors, a non-significant improvement was observed. Moreover, surgeons rated the navigation system favorable over the conventional setting and stated that the technology improved decisiveness and simplified complex procedures.

Image-guided surgery relies on 3 important steps: 1) the preoperative planning, including segmentation of the images, 2) registration of the preoperative images with the intraoperative imaging and 3) the intraoperative tracking and use of the navigation. It is important to note, that inaccuracy errors of the system may occur in the different steps. Segmentation is based on the preoperative images, of which interpretation can be challenging after neoadjuvant treatment. Also, registration of the images can introduce an error. All these possible errors could result in clinically relevant errors although this was not observed in our study. Another limitation of the currently used setup is that it can only be used in fixated target areas, which makes the technique not suitable for a wider range of moving abdominal tumors.

A different technique that is currently emerging as a contributor in surgical decision making is fluorescence imaging. With this technique, the patient is injected with a fluorescent contrast agent which will spread into the vascular and lymphatic system. With the use of a fluorescence imaging probe/camera, the operative field is illuminated and the fluorescent signal can be visualized [42]. Fluorescence imaging is currently tested and used for anastomotic perfusion assessment, sentinel lymph node mapping and tumor identification [43-46]. An advantage of fluorescence compared to imageguided navigation is that fluorescence does not require registration, thus automatically compensates for organ deformation and moving (oncological) targets. A drawback of fluorescence imaging compared to surgical navigation is that it requires injecting a fluorescent agent before or during the procedure. Moreover, fluorescence imaging has a poor penetration depth and allows only for surface imaging. Based on the strengths and weaknesses of these two techniques, combining them would be an interesting next step.

In the currently used setup, a hybrid operating room with a cone beam CT scanner is 
necessary to provide the intraoperative images that are needed for the registration. We are aware that this is not feasible in all hospitals and limits the number of hospitals able to use the navigation system. In addition, the patient and medical personal are exposed to extra radiation. To make the current navigation system available for more hospitals, an alternative registration method would be recommended. A possible solution could be registration with a tracked $3 \mathrm{D}$ ultrasound. Our research group is currently working on ultrasound registration. Preliminary results showed that surgical navigation with 3D tracked ultrasound registration has a lot of potential.

Chapter 6 showed that surgical navigation can improve patient outcome in fixated advanced rectal cancer patients. However, in the majority of patients diagnosed with rectal cancer, the tumor lesion is mobile and the position changes in relation to the pre-operative imaging. To investigate whether image-guided navigation would be possible in oncological abdominal surgery with non-fixed, mobile tumors, we performed a feasibility study. Chapter $\mathbf{7}$ describes the results of this study, in which real-time tumor tracking with an electromagnetic navigation system in rectal cancer surgery was evaluated. A tracked sensor on the tumor continuously determined the real-time position of the tumor. In total, 31 patients were included of which navigation was feasible in $93 \%$ in the test phase. No intraoperative navigation-related complications occurred and a high median accuracy of $3 \mathrm{~mm}$ was accomplished. This study demonstrated that real-time tumor tracking is feasible with the potential of a broader clinical implementation.

To make navigation suitable for a wide range of applications in surgical oncology, some adjustments in the current set up should be made. In our study, positioning and securing the tumor sensor against the rectal tumor was inconvenient and added additional time to the anesthesia time. But it was feasible since rectal tumors can easily be approached from the lumen of the bowel. However, positioning a wired sensor in an abdominal tumor before surgery is not possible. A solution for rectal cancer and other abdominal tumor locations would be the use of wireless tracked transponders, which has shown feasible in breast and prostate cancer $[47,48]$. Before rectal cancer surgery, these transponders could be implanted using a sigmoidoscopy. In other abdominal tumors, implantation could be performed CT-guided by an experienced radiologist. During surgery, no additional intraoperative imaging would be necessary and tumor movement would simply be tracked by the navigation system.

Another improvement of the system would be tracked surgical instruments. In the 
currently used setup, the pointer is the only tracked surgical instrument. Based on the feedback of the surgeons, additional tracked surgical instruments would be beneficial, which will increase the usability of the system even more. Therefore, we designed a laparoscopic tool that can be fixated on all laparoscopic surgical instruments to make the system more user-friendly. The accuracy and usability of this tool are being evaluated and the first clinical results are soon to be expected.

The results of Chapter 7 showed that real-time tumor tracking with image-guided navigation is feasible and safe in rectal cancer surgery. However, it remains unclear whether this technique will improve patient outcome. A randomized controlled trial comparing patients operated with and without the navigation system is recommended to examine the efficacy of the technique on resection margin rates. 


\section{References}

1. Bujko, K., L.Wyrwicz, A. Rutkowski, et al., Long-course oxaliplatin-based preoperative chemoradiation versus $5 \times 5$ Gy and consolidation chemotherapy for cT4 or fixed cT3 rectal cancer: results of a randomized phase III study. Ann Oncol, 2016. 27(5): p. 834-42.

2. Richtlijnen database - Colorectaal carcinoom (CRC). 29-10-2019 20-01-2020]; Available from: https:// richtlijnendatabase.nl/richtlijn/colorectaal_carcinoom_crc/startpagina_-_crc.html\#algemeen.

3. Nierop, P.M.H., M. Verseveld, B. Galjart, et al., The liver-first approach for locally advanced rectal cancer and synchronous liver metastases. Eur J Surg Oncol, 2019. 45(4): p. 591-596.

4. Jegatheeswaran, S., J.M. Mason, H.C. Hancock, et al., The liver-first approach to the management of colorectal cancer with synchronous hepatic metastases: a systematic review. JAMA Surg, 2013. 148(4): p. 385-391.

5. Adam, R., A. De Gramont, J. Figueras, et al., The oncosurgery approach to managing liver metastases from colorectal cancer: multidisciplinary international consensus. Oncologist, 2012. 17(10): p. 1225.

6. Maas, M., P.J. Nelemans, V. Valentini, et al., Long-term outcome in patients with a pathological complete response after chemoradiation for rectal cancer: a pooled analysis of individual patient data. 2010. 11(9): p. 835-844.

7. Al-Sukhni, E., K. Attwood, D.M. Mattson, et al., Predictors of pathologic complete response following neoadjuvant chemoradiotherapy for rectal cancer. Ann Surg Oncol. 2016. 23(4): p. 1177-1186.

8. van der Valk, M.J.M., D.E. Hilling, E. Bastiaannet, et al., Long-term outcomes of clinical complete responders after neoadjuvant treatment for rectal cancer in the International Watch \& Wait Database (IWWD): an international multicentre registry study. Lancet, 2018. 391(10139): p. 2537-2545.

9. Renehan, A.G., L. Malcomson, R. Emsley, et al., Watch-and-wait approach versus surgical resection after chemoradiotherapy for patients with rectal cancer (the OnCoRe project): a propensity-score matched cohort analysis. Lancet Oncol, 2016. 17(2): p. 174-183.

10. Appelt, A.L., J. Ploen, H. Harling, et al., High-dose chemoradiotherapy and watchful waiting for distal rectal cancer: a prospective observational study. Lancet Oncol, 2015. 16(8): p. 919-27.

11. Wong, R., D. Cunningham, Y. Barbachano, et al., A multicentre study of capecitabine, oxaliplatin plus bevacizumab as perioperative treatment of patients with poor-risk colorectal liver-only metastases not selected for upfront resection. Ann Oncol, 2011. 22(9): p. 2042-8.

12. Folprecht, G., T. Gruenberger, W.O. Bechstein, et al., Tumour response and secondary resectability of colorectal liver metastases following neoadjuvant chemotherapy with cetuximab: the CELIM randomised phase 2 trial. Lancet Oncol, 2010. 11(1): p. 38-47.

13. Jackson, W.C., Y. Tao, M. Mendiratta-Lala, et al., Comparison of Stereotactic Body Radiation therapy and Radiofrequency Ablation in the Treatment of Intrahepatic Metastases. International Journal of Radiation Oncology*Biology*Physics, 2018. 100(4): p. 950-958.

14. Franzese, C., T. Comito, E. Clerici, et al., Liver metastases from colorectal cancer: propensity scorebased comparison of stereotactic body radiation therapy vs. microwave ablation. J Cancer Res Clin Oncol, 2018.

15. Palma, D.A., R. Olson, S. Harrow, et al., Stereotactic ablative radiotherapy versus standard of care palliative treatment in patients with oligometastatic cancers (SABR-COMET): a randomised, phase 2, open-label trial. Lancet, 2019. 393(10185): p. 2051-2058.

16. Ruers, T., F. Van Coevorden, C.J. Punt, et al., Local Treatment of Unresectable Colorectal Liver Metastases: Results of a Randomized Phase II Trial. J Natl Cancer Inst, 2017. 109(9).

17. Goodman, B.D., E.M. Mannina, S.K. Althouse, et al., Long-term safety and efficacy of stereotactic body radiation therapy for hepatic oligometastases. Pract Radiat Oncol, 2016. 6(2): p. 86-95.

18. Lee, M.T., J.J. Kim, R. Dinniwell, et al., Phase I study of individualized stereotactic body radiotherapy of liver metastases. J Clin Oncol, 2009. 27(10): p. 1585-91.

19. Høyer, M., A. Swaminath, S. Bydder, et al., Radiotherapy for liver metastases: a review of evidence. Int J Radiat Oncol Biol Phys, 2012. 82(3): p. 1047-1057.

20. Mahadevan, A., O. Blanck, R. Lanciano, et al., Stereotactic Body Radiotherapy (SBRT) for liver 
metastasis - clinical outcomes from the international multi-institutional RSSearch(R) Patient Registry. Radiat Oncol, 2018. 13(1): p. 26.

21. Schulz, A., E. Viktil, J.C. Godt, et al., Diagnostic performance of CT, MRI and PET/CT in patients with suspected colorectal liver metastases: the superiority of MRI. Acta Radiologica, 2016. 57(9): p. 10401048.

22. Hu, C.-Y., C.E. Bailey, Y.N. You, et al., Time Trend Analysis of Primary Tumor Resection for Stage IV Colorectal Cancer: Less Surgery, Improved SurvivalTrend Analysis of Primary Tumor Resection for CRCTrend Analysis of Primary Tumor Resection for CRC. JAMA Surgery, 2015. 150(3): p. 245-251.

23. Simillis, C., E. Kalakouti, T. Afxentiou, et al., Primary Tumor Resection in Patients with Incurable Localized or Metastatic Colorectal Cancer: A Systematic Review and Meta-analysis. World J Surg, 2019. 43(7): p. 1829-1840.

24. van der Pool, A.E., R.A. Damhuis, J.N. ljzermans, et al., Trends in incidence, treatment and survival of patients with stage IV colorectal cancer: a population-based series. Colorectal Dis, 2012. 14(1): p. 56-61.

25. Elferink, M.A., K.P. de Jong, J.M. Klaase, et al., Metachronous metastases from colorectal cancer: a population-based study in North-East Netherlands. Int J Colorectal Dis, 2015. 30(2): p. 205-12.

26. Foxtrot Collaborative, G., Feasibility of preoperative chemotherapy for locally advanced, operable colon cancer: the pilot phase of a randomised controlled trial. Lancet Oncol, 2012. 13(11): p. 115260.

27. Nelson, H., N. Petrelli, A. Carlin, et al., Guidelines 2000 for colon and rectal cancer surgery. J Natl Cancer Inst, 2001. 93(8): p. 583-96.

28. Tohme, S., R.L. Simmons and A. Tsung, Surgery for Cancer: A Trigger for Metastases. Cancer research, 2017. 77(7): p. 1548-1552.

29. Seymour, M.T., D. Morton and o.b.o.t.I.F.T. Investigators, FOxTROT: an international randomised controlled trial in 1052 patients (pts) evaluating neoadjuvant chemotherapy (NAC) for colon cancer. 2019.37(15_suppl): p. 3504-3504.

30. Bonjer, H.J., C.L. Deijen, G.A. Abis, et al., A Randomized Trial of Laparoscopic versus Open Surgery for Rectal Cancer. N Engl J Med, 2015. 372(14): p. 1324-1332.

31. Jayne, D.G., P.J. Guillou, H. Thorpe, et al., Randomized trial of laparoscopic-assisted resection of colorectal carcinoma: 3-year results of the UK MRC CLASICC Trial Group. 2007. 25(21): p. 3061-3068.

32. Komenaka, I.K., K. Giffard, J. Miller, et al., COLOR: a randomized clinical trial comparing laparoscopic and open resection for colon cancer. Dig Surg, 2000. 17(6): p. 617-622.

33. Rickles, A.S., D.W. Dietz, G.J. Chang, et al., High Rate of Positive Circumferential Resection Margins Following Rectal Cancer Surgery: A Call to Action. Annals of surgery, 2015. 262(6): p. 891-898.

34. Bonjer, H.J., C.L. Deijen, G.A. Abis, et al., A Randomized Trial of Laparoscopic versus Open Surgery for Rectal Cancer. 2015. 372(14): p. 1324-1332.

35. Jaafar, H., Intra-operative frozen section consultation: concepts, applications and limitations. The Malaysian journal of medical sciences : MJMS, 2006. 13(1): p. 4-12.

36. Gomes, R.M., M. Bhandare, A. Desouza, et al., Role of intraoperative frozen section for assessing distal resection margin after anterior resection. Int J Colorectal Dis, 2015. 30(8): p. 1081-9.

37. Krand, O., T. Yalti, G. Tellioglu, et al., Use of Smooth Muscle Plasty After Intersphincteric Rectal Resection to Replace a Partially Resected Internal Anal Sphincter: Long-Term Follow-Up. Dis Colon Rectum, 2009. 52(11): p. 1895-1901.

38. Khoury, W., W. Abboud, D. Hershkovitz, et al., Frozen section examination may facilitate reconstructive surgery for mid and low rectal cancer. J Surg Oncol, 2014. 110(8): p. 997-1001.

39. Ying, M., S. Zhuo, G. Chen, et al., Real-time noninvasive optical diagnosis for colorectal cancer using multiphoton microscopy. 2012. 34(3): p. 181-185.

40. Li, Y., Z. Zhu, J.J. Chen, et al., Multimodal endoscopy for colorectal cancer detection by optical coherence tomography and near-infrared fluorescence imaging. Biomedical optics express, 2019. 10(5): p. 2419-2429. 
41. Jenkins, C.A., P.D. Lewis, P.R. Dunstan, et al., Role of Raman spectroscopy and surface enhanced Raman spectroscopy in colorectal cancer. World journal of gastrointestinal oncology, 2016. 8(5): p. 427-438.

42. Leblond, F., S.C. Davis, P.A. Valdés, et al., Pre-clinical whole-body fluorescence imaging: Review of instruments, methods and applications. Journal of Photochemistry and Photobiology B: Biology, 2010. 98(1): p. 77-94.

43. Keller, D.S., T. Ishizawa, R. Cohen, et al., Indocyanine green fluorescence imaging in colorectal surgery: overview, applications, and future directions. The Lancet Gastroenterology \& Hepatology, 2017. 2(10): p. 757-766.

44. Nagaya, T., Y.A. Nakamura, P.L. Choyke, et al., Fluorescence-Guided Surgery. Frontiers in oncology, 2017. 7: p. 314-314.

45. Blanco-Colino, R. and E. Espin-Basany, Intraoperative use of ICG fluorescence imaging to reduce the risk of anastomotic leakage in colorectal surgery: a systematic review and meta-analysis. Tech Coloproctol, 2018. 22(1): p. 15-23.

46. Ankersmit, M., H.J. Bonjer, G. Hannink, et al., Near-infrared fluorescence imaging for sentinel lymph node identification in colon cancer: a prospective single-center study and systematic review with meta-analysis. Tech Coloproctol, 2019. 23(12): p. 1113-1126.

47. Willoughby, T.R., P.A. Kupelian, J. Pouliot, et al., Target localization and real-time tracking using the Calypso 4D localization system in patients with localized prostate cancer. International Journal of Radiation Oncology* Biology* Physics, 2006. 65(2): p. 528-534.

48. Janssen, N., R. Eppenga, M.-J.V. Peeters, et al., Real-time wireless tumor tracking during breast conserving surgery. International Journal of Computer Assisted Radiology and Surgery 2018. 13(4): p. 531-539. 



\section{Appendices}

Nederlandse samenvatting

PhD Portfolio

Dankwoord

Curriculum vitae 


\section{Nederlandse samenvatting}

Colorectaal carcinoom is, met circa 14.000 nieuwe gevallen per jaar, een van de meest voorkomende vormen van kanker in Nederland. De standaardbehandeling voor deze patiënten is chirurgische resectie, al dan niet in combinatie met radio- en/of systemische therapie. Het type behandeling is sterk afhankelijk van de uitgebreidheid van de ziekte bij diagnose. Vooral bij patiënten met gemetastaseerde ziekte blijft het optimale behandelschema controversieel. Bij het merendeel van de patiënten $( \pm 80 \%)$ in Nederland wordt een chirurgische resectie verricht, waarbij het radicaal verwijderen van de tumor zeer bepalend is voor de prognose. Helaas wordt er tot op heden bij zo'n $10 \%$ van de patiënten een irradicale resectie verricht. Om dit te verbeteren zouden aanvullende intra-operatieve technieken van toegevoegde waarde kunnen zijn.

Het eerste deel van dit proefschrift focust op nieuwe toepassingsmogelijkheden van reeds bestaande behandelmodaliteiten voor patiënten met gemetastaseerd colorectaal carcinoom. Hierbij is gekeken naar drie behandelmethoden: de combinatie van radiotherapie met systemische therapie bij patiënten met een curatieve opzet; de toepassing van radiotherapie bij inoperabele levermetastasen; en neoadjuvante systemische therapie om de groei van de levermetastasen te voorkomen nadat de primaire tumor is verwijderd.

De focus van het tweede deel van dit proefschrift ligt op nieuwe technieken die tot doel hebben de radicaliteit van colorectale chirurgie te verbeteren. In dit deel is onderzocht of hyperspectrale imaging een geschikte techniek is om tumor van gezond weefsel te onderscheiden met het uiteindelijke doel de hoeveelheid irradicale resecties te verlagen. Verder is de toepassing van een geavanceerd navigatiesysteem tijdens de operatie getest. Hierbij is gekeken of beeldgestuurde navigatie de chirurgische uitkomsten van patiënten met vergevorderd rectumcarcinoom kan verbeteren. Ook is onderzocht of de techniek toepasbaar is in mobiele rectumtumoren.

\section{Hoofdstuk 2}

Het optimale behandelschema van patiënten met gemetastaseerd rectumcarcinoom is tot op heden onduidelijk. Kortdurende radiotherapie ( $5 \times 5 \mathrm{~Gy}$ ) met aansluitend systemische therapie, gevolgd door lokale behandeling van alle tumorlocaties, combineert lokale controle van de primaire tumor met vroege behandeling van de afstandsmetastasen. In hoofdstuk 2 wordt de haalbaarheid en effectiviteit van dit schema in de dagelijkse praktijk geëvalueerd. 
In dit retrospectieve onderzoek werden patiënten geëvalueerd met een rectumcarcinoom en (potentieel) curabele synchrone levermetastasen met of zonder extrahepatische metastasen, die werden behandeld tussen 2010 en 2015 in 8 tertiaire verwijscentra in Nederland. De uitkomstmaten waren: voltooiing van het gehele behandelschema, symptoombestrijding, progressie vrije overleving en algehele overleving. Voltooiing van het gehele behandelschema werd gedefinieerd als het ondergaan van een kort schema radiotherapie ( $5 \times 5 \mathrm{~Gy})$, tenminste twee cycli systemische therapie en daaropvolgende lokale behandeling van alle aanwezige tumorlocaties.

In totaal werden 169 patiënten geïncludeerd met een mediane follow-upperiode van 50 maanden ( 2 - 89 maanden). Voltooiing van het gehele behandelschema werd bereikt in $66 \%$ van de patiënten. Adequate symptoombestrijding van de rectumtumor werd geobserveerd bij $87 \%$ van de patiënten. De 3-jaar progressie vrije overleving en algehele overleving waren respectievelijk $24 \%$ en $49 \%$ ( $95 \%$ betrouwbaarheidsinterval (BI): 17 - 32 en 40 - 57). In multivariabele analyse was de hoeveelheid levermetastasen ( $\leq$ 4 laesies) een positieve voorspeller voor zowel het voltooien van het behandelschema als de algehele overleving. Mediane algehele overleving van patiënten waarbij het gehele schema voltooid werd was 52 maanden vs. 15 maanden in patiënten waarbij het behandelschema niet werd voltooid $(p<0.001)$.

Deze resultaten laten zien dat twee derde van de patiënten met potentieel curatief stadium IV rectumcarcinoom in staat is de behandeling met kortdurende radiotherapie, systemische therapie en aansluitend lokale behandeling van alle tumorlocaties te voltooien. Adequate symptoombestrijding wordt in het grootste deel van de patiënten behaald. Voltooiing van het behandelschema was significant gecorreleerd met een betere algehele overleving.

\section{Hoofdstuk 3}

In hoofdstuk 3 wordt de toepassing van radiotherapie als behandeloptie voor levermetastasen geëvalueerd. In het verleden was de rol van radiotherapie in de behandeling van levermetastasen klein vanwege het risico op radiatie geïnduceerde leverziekte (RILD). In de afgelopen jaren is de techniek enorm verbeterd en wordt radiotherapie in toenemende mate gebruikt wanneer chirurgische resectie niet mogelijk is. In dit hoofdstuk wordt de effectiviteit en veiligheid van hoge versus lage dosis radiotherapie van levermetastasen vergeleken. 
Patiënten die tussen 2009 en 2017 waren behandeld met stereotactische radiotherapie in het Antoni van Leeuwenhoek werden retrospectief geëvalueerd en geïncludeerd. Patiënten werden verdeeld in een lage dosis groep <100 Gray (Gy) en een hoge dosis groep $\geq 100$ Gy biologisch effectieve dosis (BED). De mediane voorgeschreven bestralingsdosis van deze groepen was respectievelijk 37,5 Gy (bereik: 36 - 45 Gy) en 60 Gy (bereik: 51 - 60 Gy) in 3-5 fracties. De uitkomstmaten waren: lokale controle binnen het hoge dosis bestralingsgebied, toxiciteit en algehele overleving.

In totaal werden 90 patiënten met 97 levermetastasen van verschillende origine (77\% colorectaal carcinoom) geïncludeerd: 33 patiënten in de lage dosis groep en 57 patiënten in de hoge dosis groep. De mediane follow-up was 29 maanden. De 2-jaar lokale controle in de lage dosis groep was 60\% (95\% BI: 41 -80\%) en in de hoge dosis groep 90\% (95\% BI: 80 - 100\%) ( $p=0.004)$. Graad 3 toxiciteit waarbij ziekenhuisopname was vereist kwam in de lage dosis en hoge dosis groep voor bij respectievelijk $7 \%$ en $2 \%$ van de patiënten. Graad 4 en 5 toxiciteit werd niet geobserveerd. De 2-jaar algehele overleving in de lage en hoge dosis groep waren respectievelijk 48\% (95\% BI: 32 $65 \%$ ) en $85 \%$ (95\% BI: 73 - 97\%) ( $p=0.007)$. Bestralingsdosis en tumorvolume waren beide significante voorspellers voor lokale controle (hazard ratio (HR): $3.61 ; p=0.017$ en HR: $1.01 ; p=0.005$ ) en algehele overleving (HR: 2.38; $p=0.005$ en HR: $1.01 ; p=$ $<0.0001)$.

Uit deze studie kan geconcludeerd worden dat hoge dosis radiotherapie significant betere lokale controle biedt dan lage dosis zonder toename van de toxiciteit. Indien chirurgische resectie niet mogelijk is, biedt hoge dosis stereotactische bestraling een effectief en veilig alternatief bij de behandeling van levermetastasen.

\section{Hoofdstuk 4}

Hoofdstuk 4 bevat een prospectief gerandomiseerde pilotstudie waarin het effect van verschillende soorten neoadjuvante systemische therapie bij patiënten met colorectale levermetastasen wordt geëvalueerd. Volgens de huidige behandelrichtlijn worden patiënten met coloncarcinoom behandeld met chirurgische resectie gevolgd door adjuvante systemische therapie. Helaas ontwikkelt, ondanks de systemische behandeling, 20\% van de patiënten afstandsmetastasen nadat de colontumor is verwijderd. Mogelijk veroorzaakt de resectie van de primaire tumor een exponentiële groei van de levermetastasen. De timing van de systemische therapie kan daarom in twijfel worden getrokken. Deze studie evalueert of behandeling met systemische therapie voorafgaand aan resectie van de colorectale tumor de uitgroei van 
levermetastasen, veroorzaakt door primaire tumorresectie, kan voorkomen.

Patiënten werden gerandomiseerd in de controlegroep (groep A) of in één van de drie behandelgroepen (groep B, C en D). De controlegroep ontving geen neoadjuvante behandeling voordat de primaire tumor werd verwijderd. De behandelgroepen ontvingen wel neoadjuvante therapie (groep B: bevacizumab; groep C: capecitabine/ oxaliplatin (CAPOX); groep D: CAPOX-bevacizumab) voordat de primaire tumor werd verwijderd. Op de dag van de primaire tumor resectie werden een CT-scan en PETCT scan, bloedafname en een leverbiopt verricht. Vier weken na de resectie werden deze scans, de bloedafname en het lever biopt herhaald. De uitkomstmaten waren: tumorvolume op CT, FDG-opname op PET-CT en CEA-waarden in het bloed. Secundair werd histopathologisch onderzoek verricht op leverbiopten, waarbij werd gekeken naar het effect van de primaire tumorresectie op de proliferatie en apoptose in de metastasen.

In totaal werden 47 patiënten gerandomiseerd. In groep A werd een significante groei ( $p=0.028$ ) en een verhoogde FDG-opname $(p=0.022)$ van de levermetastasen waargenomen na de primaire tumor resectie ten opzichte van de scans vóór de resectie. In de behandelgroepen was geen significant verschil in tumorgrootte of FDGopname zichtbaar nadat de primaire tumor was verwijderd. Verder lieten CEA-waardes een niet-significante stijging zien in groep $A(p=0.068)$ en een significante daling in groep $D(p=0.003)$. In zowel de controle groep als de behandelgroepen werden in het histopathologisch onderzoek geen significante verschillen geobserveerd qua proliferatie en apoptose na de primaire tumor resecties.

De data in deze pilotstudie tonen aan dat primaire tumor resectie exponentiële groei van levermetastasen induceert en dat neoadjuvante systemische therapie deze groei kan voorkomen. Nader onderzoek met een grotere patiëntenpopulatie is echter noodzakelijk om eventuele aanpassingen in de huidige behandelrichtlijn voor patiënten met coloncarcinoom te rechtvaardigen.

\section{Hoofdstuk 5}

Het radicaal verwijderen van rectumtumoren kan voor chirurgen uitdagend zijn. Vooral bij patiënten waarbij de anatomische vlakken verstoord zijn door ingroei van tumor weefsel of bestralingseffecten worden hoge percentages (10\%) irradicale resecties gezien. Om de resectiemarges te verbeteren, zou een intra-operatieve techniek die tumor van gezond weefsel kan onderscheiden van toegevoegde waarden kunnen 
zijn. In hoofdstuk 5 staat de toepassing van hyperspectrale imaging met dit doeleinde centraal.

In deze ex vivo studie is hyperspectrale imaging afgezet tegen de huidige gouden standaard: histopathologische beoordeling van het weefsel onder een microscoop. $\mathrm{Na}$ een colorectale resectie werden drie typen weefsels bij de pathologie verkregen: vet, gezonde darmwand en tumor. De weefsels werden onder twee hyperspectrale camera's gelegd: een met het visuele golflengte gebied en een met het nabije infrarode golflengtegebied. De weefsels werden daarna volgens het standaard pathologie protocol verwerkt. De hyperspectrale afbeeldingen werden op de verkregen pathologie coupes geregistreerd zodat er van iedere pixel in de hyperspectrale afbeelding een pathologische classificatie bekend was. Er werden twee classificatie algoritmes gebruikt om de verschillende weefseltypen te onderscheiden: een kwadratische classificatie om vet te onderscheiden en een support vector machine voor de classificatie van tumor en gezond darmweefsel.

In totaal werden er van 32 patiënten afbeeldingen gemaakt met beide hyperspectrale camera's. Wanneer de data van beide camera's werden gebruikt voor weefselclassificatie, resulteerde dit in een algehele nauwkeurigheid van $88 \%$ ( $\pm 13 \%$ ) om de drie weefseltypen van elkaar te onderscheiden. Wanneer enkel de data van het visuele golflengtegebied of het nabije infrarode golflengte gebied werden gebruikt, verminderde de nauwkeurigheid naar respectievelijk 67\% ( $\pm 19 \%)$ en $83 \%$ ( $\pm 12 \%)$.

Hieruit kan worden geconcludeerd dat hyperspectrale imaging gebruikt kan worden om gezond weefsel van tumor weefsel te onderscheiden in colorectale tumoren. Voor optimale resultaten moeten beiden golflengtegebieden gebruikt worden. Vervolgonderzoek moet deze resultaten valideren in een in vivo setting voordat deze techniek klinisch gebruikt kan worden.

\section{Hoofdstuk 6}

Zoals eerder beschreven, blijft het percentage irradicale resecties bij oncologische rectumchirurgie hoog. Het percentage irradicale resectiemarges bij patiënten met lokaal vergevorderd rectumcarcinoom en patiënten met recidief rectumcarcinoom is respectievelijk $15 \%$ en $50 \%$. In het vorige hoofdstuk is de toepassing van hyperspectrale imaging besproken. Een alternatieve techniek om chirurgische radicaliteit te verbeteren zou beeldgestuurde intra-operatieve navigatie kunnen zijn. Deze techniek wordt reeds gebruikt in de neuro- en orthopedische chirurgie. Beeldgestuurde intra-operatieve 
navigatie stelt de chirurg in staat om de chirurgische instrumenten ten opzichte van de omliggende anatomische structuren te zien tijdens de operatie. Hierdoor kunnen de tumormarges met meer zekerheid worden bepaald en kunnen gezonde omliggende structuren gespaard worden. In hoofdstuk 6 worden de eerste klinische resultaten van een nieuw beeldgestuurd navigatiesysteem gebruikt tijdens rectumresecties gerapporteerd.

In deze prospectieve studie werden patiënten met lokaal vergevorderde rectumcarcinoom of recidief rectumcarcinoom geïncludeerd. Preoperatief werd een patiënt-specifiek driedimensionaal (3D) model gemaakt op basis van CT- en MRI-scans. In de operatiekamer werd een intra-operatieve CT-scan gemaakt. De intra-operatieve beelden werden op basis van de botstructuren gelinkt aan het 3D-model. Een elektromagnetisch tracking systeem kon, met behulp van elektromagnetische sensoren op de patiënt, continue de positie van de patiënt bepalen. Tijdens de operatie was een elektromagnetische aanwijspen beschikbaar om de registratienauwkeurigheid te valideren en om de anatomie en tumorlocatie te beoordelen. De primaire uitkomstmaat van deze studie was pathologisch radicale resectiemarges. De klinische resultaten werden vergeleken met resultaten van patiënten uit een historisch cohort. Dit historisch cohort bevatte patiënten die geopereerd waren aan een rectumcarcinoom zonder navigatiesysteem tussen 2009 en 2015. Verdere uitkomstwaarden waren veiligheid en bruikbaarheid van het navigatiesysteem, waarbij de bruikbaarheid werd beoordeeld met behulp van vragenlijsten ingevuld door de chirurg.

Tussen februari 2016 en september 2019 werden in het Antoni van Leeuwenhoek 33 patiënten geopereerd met het navigatiesysteem; 14 patiënten met lokaal vergevorderde rectumcarcinoom en 19 patiënten met recidief van het rectumcarcinoom. Bij patiënten met lokaal vergevorderd rectumcarcinoom werden radicale resectiemarges gezien in 14 van de 15 patiënten (93\%). Bij patiënten met een recidief was dit in 15 van de 19 patiënten (79\%) het geval. Er werden geen navigatie-gerelateerde complicaties waargenomen. Verder verklaarden chirurgen dat het navigatiesysteem de besluitvaardigheid verbeterde en van toegevoegde waarde was bij het lokaliseren van tumoren. In het historische cohort werden 142 patiënten geïncludeerd. Een radicale resectie werd bereikt bij $84 \%$ (85/101) van de patiënten met lokaal vergevorderde rectumcarcinoom en bij 49\% (20/41) van de patiënten met een recidief. Bij patiënten met een recidief werd een significant verschil in radicale resecties waargenomen tussen de beeldgestuurde navigatiegroep en het historisch cohort ( $p=0.047$ ). De hoeveelheid radicale marges bij lokaal vergevorderd rectumcarcinoom was niet 
significant verschillend tussen beide groepen $(p=0.690)$.

Deze studie toont aan dat het gebruik van intra-operatieve navigatie bij lokaal vergevorderd primair en recidief rectumcarcinoom veilig en effectief is. Bij patiënten met recidief rectumcarcinoom worden significant meer radicale resecties bereikt met het navigatiesysteem. Chirurgen geven aan dat het navigatiesysteem complexe operaties vereenvoudigt en de besluitvaardigheid vergroot.

\section{Hoofdstuk 7}

In hoofdstuk 7 wordt opnieuw de toepassing van beeldgestuurde navigatie geëvalueerd, maar nu bij patiënten met mobiele rectumtumoren. Het navigatiesysteem beschreven in hoofdstuk 6 heeft als voorwaarde dat het gebied van interesse een rigide anatomie heeft tijdens de operatie. Dit is het geval bij patiënten met vergevorderd rectumcarcinoom waarbij de tumor door anatomische vlakken groeit. Maar bij de meeste patiënten met minder ver gevorderd rectumcarcinoom, verandert de positie van het rectum - en daarmee de tumor - tijdens de operatie. Om van chirurgische navigatie in deze omstandigheden een nuttige techniek te maken, is real-time intraoperatieve informatie nodig om deze bewegingen te kunnen volgen.

Om dit mogelijk te maken werd een elektromagnetische sensor toegevoegd aan het reeds bestaande navigatiesysteem zoals beschreven in hoofdstuk 6. Deze sensor werd op de operatiekamer door de chirurg tegen de rectum tumor geplaatst voordat de intraoperatieve CT-scan werd verricht. De registratie ging vervolgens in twee stappen: eerst werd een rigide registratie van de intra-operatieve beelden en het 3D-model verricht. In aanvulling daarop werd een aparte tumorregistratie gedaan waardoor bewegingen van de tumor tijdens de operatie continue geobserveerd konden worden. De studie was opgedeeld in twee fases. In de eerste fase werd de haalbaarheid en veiligheid van de nieuwe opstelling met real-time tumor tracking geëvalueerd. In de tweede fase, de testfase, werd de nauwkeurigheid van de real-time tracking bepaald.

In de eerste fase werden 15 patiënten met rectumcarcinoom geïncludeerd, waarvan 11 patiënten (73\%) met real-time tumor tracking konden worden geopereerd. Bij 4 patiënten was tumor tracking niet mogelijk doordat de sensor was beschadigd tijdens het bevestigen aan de tumor. Verder bleek in de eerste fase dat de tumorregistratie moeilijk was. Om dit te verbeteren kregen patiënten in de testfase, voor de preoperatieve CT-scan, goudmarkers geplaatst in de tumor met behulp van een sigmoïdoscopie. Met behulp van de goudmarkers kon de tumorregistratie gemakkelijk verricht worden. 
In de testfase werden 16 patiënten geïncludeerd, waarvan 14 patiënten met het navigatiesysteem werden geopereerd. Bij alle 14 patiënten was het mogelijk om de bewegingen van de tumor te tracken tijdens de operatie. De mediane nauwkeurigheid van het systeem was $3 \mathrm{~mm}$ (interkwartielafstand: 2 - $10 \mathrm{~mm}$ ).

Deze studie is, voor zover bekend, de eerste studie waarbij real-time tumor tracking van rectumtumoren in een grotere patiëntenpopulatie is geëvalueerd. De toepassing van deze techniek is haalbaar en veilig met een hoge nauwkeurigheid. Deze techniek stelt oncologische chirurgen in staat om real-time nauwkeurige informatie over de locatie van de tumor te verkrijgen, evenals kritische anatomische informatie. Nader onderzoek moet uitwijzen of deze techniek chirurgische resectiemarges verbetert. 


\section{PhD Portfolio}

PhD Period: August 2017 - March 2020

PhD supervisors: prof. dr. T.J.M. Ruers and dr. K.F.D. Kuhlmann

\section{List of publications}

High versus low dose Stereotactic Body Radiation Therapy for hepatic metastases

E.N.D. Kok, E.P.M. Jansen, B.C. Heeres, N.F.M. Kok, T. Janssen, E. van Werkhoven, F.R.K. Sanders, T.J.M. Ruers, M.E. Nowee, K.F.D. Kuhlmann

Clinical Translation Radiation Oncology (2019): 20, 45-50. https://doi.org/10.1016/j. ctro.2019.11.004

Hyperspectral imaging for tissue classification, a way towards smart laparoscopic colorectal surgery

E.N.D. Kok, E.J.M. Baltussen, S.G. Brouwer de Koning, J. Sanders, A.G.J. Aalbers, N.F.M. Kok, G.L. Beets, C.C. Flohil, S.C. Bruin, K.F.D. Kuhlmann, H.J.C.M. Sterenborg, T.J.M. Ruers JournalofBiomedicalOptics(2019):24(1): 1-9.https://doi.org/10.1117/1.JBO.24.1.016002

Multicentre study of short-course radiotherapy, systemic therapy and resection/ ablation for stage IV rectal cancer

E.N.D. Kok, K. Havenga, P.J. Tanis, J.H.W. de Wilt, J. Hagendoorn, F.P. Peters, J. Buijsen, H.J.T. Rutten, K.F.D. Kuhlmann and The Dutch Stage IV Rectal Cancer group British Journal of Surgery (2020): 107(5): 537-545. https://doi.org/10.1002/bjs.11418

Accurate surgical navigation with real-time tumor tracking in cancer surgery

E.N.D. Kok, R. Eppenga, K.F.D. Kuhlmann, H.C. Groen, R. van Veen, J.M. van Dieren, T.R. de Wijkerslooth, M. van Leerdam, D.M.J. Lambregts, W. J. Heerink, N.J. Hoetjes, O. Ivashchenko, G.L. Beets, A.G.J. Aalbers, J. Nijkamp, T.J.M. Ruers

npj precision oncology (2020): 4, 8 (2020). https://doi.org/10.1038/s41698-020-0115-0

Association of image-guided navigation with complete resection in patients with locally advanced primary and recurrent rectal cancer

E.N.D. Kok, R. van Veen, H.C. Groen, W.J. Heerink, N.J. Hoetjes, E. van Werkhoven, G.L. Beets, A.G.J. Aalbers, K.F.D. Kuhlmann, J. Nijkamp, T.J.M. Ruers

JAMA Network Open (2020); 3(6):e208522. https://doi.org/10:1001/jamanetworkopen. 2020.8522 
Accelerated growth of synchronous colorectal liver metastases after primary tumor resection: effects of neoadjuvant therapy

K.F.D. Kuhlmann, E.N.D. Kok, M. Sinasappel, O. Ivashchenko, J. Sanders, I. Hofland, J.D. van Buul, N.F.M. Kok, C. Groothuizen, T.J.M. Ruers

In preparation, 2020

An alternative method for evaluation of 18-FDG uptake in synchronous colorectal liver metastasis

M. Sinaasappel, E.N.D. Kok, M. Donswijk, K.F.D. Kuhlmann, T.J.M. Ruers

In preparation, 2020

Image-Guided Navigation For Locally Advanced Primary and Locally Recurrent Rectal Cancer: Evaluation of its Early Cost-Effectiveness

M.A. Lindenberg*, A. Kramer*, E.N.D. Kok, V.P. Retel, G.L. Beets, T.J.M. Ruers, W.H. van Harten. *Shared first author

Submitted, 2020

\section{Conferences and presentations}

2018 Spie Photonics West, San Francisco

2018 Chirurgendagen, Veldhoven (oral)

2018 International Hepato-Pancreato-Biliary Association, Genève (oral)

2018 Regionaal refereeravond region 2, Amsterdam (oral)

2019 European Society of Surgical Oncology, Budapest (oral)

2019 Chirurgendagen, Veldhoven (oral)

2019 European Society of Surgical Oncology, Rotterdam (poster)

2019 Wetenschapsdag chirurgie regio 1\&2, Amsterdam (oral)

\section{Courses}

2017 Matlab course (http://www.imc.tue.nl/)

2017 Machine learning course (https://www.coursera.org)

2018 Chirurgenweek, AvL

2018 OOA retreat, Renesse

2018 Basic Medical Statistics Course, OOA

2018 How to write high impact papers and what to do when it gets rejected, OOA

2019 Writing a scientific paper, OOA

2019 Good Clinical Practice, AvL 


\section{Dankwoord}

Prof. dr. Ruers, beste Theo, bedankt voor de mogelijkheden die je me hebt gegeven tijdens mijn promotietraject. In het begin van mijn PhD hebben we niet veel samen gewerkt maar aan het eind des te meer. Bedankt dat je zo actief betrokken was bij mijn artikelen toen ik het 't meeste nodig had. Ik heb bewondering voor je eeuwige optimisme! Je weet altijd wel iets positiefs te vinden!

Dr. Kuhlmann, beste Koert, toen ik als student de keuze kreeg een promotietraject bij jou en Theo of elders in het AvL te doen stond ik enorm in dubio. Jouw enthousiasme heeft me over de streep getrokken en wat ben ik blij dat ik die beslissing destijds zo heb genomen! Bedankt voor de intensieve begeleiding en je altijd opbouwende feedback. Je kritische blik heeft mijn artikelen naar een hoger niveau getild. Ik heb ontzettend veel van je geleerd, zowel op professioneel als persoonlijk vlak. Ik hoop dat we elkaar nog vaak zullen tegenkomen in de toekomst!

Geachte leden van de promotiecommissie, hartelijk dank voor uw interesse en tijd om dit proefschrift kritisch te lezen en te beoordelen. Prof. dr. Beets, beste Geerard, bedankt voor je ontspannen houding bij alle handelingen die voor deze studies verricht moesten worden. Ik vond het fijn dat je altijd naar mijn presentaties kwam kijken op congressen om me te steunen.

Beste collega's en oud-collega's, door jullie gezelligheid ben ik de afgelopen 2,5 jaar met zoveel plezier naar mijn werk gegaan. Natasja, ik begin met jou want jij was in mijn eerste maanden het zonnetje in ons hok zonder daglicht. Wat hebben we samen gelachen ('snaaacckksss') en ik ben dan ook blij dat we vriendinnetjes zijn geworden. Jullie opzoeken in Kingston was de kers op de taart. Ruben, met jou heb ik lief en leed gedeeld. Bedankt voor alle keren dat je me hebt geholpen met de navigatiemodellen, $i k$ ben er nooit écht handig in geworden. Bij elkaar op één kamer zitten heeft onze efficiëntie waarschijnlijk niet altijd bevorderd maar ik had onze vroege ochtend gesprekjes niet willen missen. Matteo, my favourite Italian collegue. Thank you for always taking time to help me when I was struggeling with my sentences. I will always remember you as the collegue who tried to get high on sterilium after lunch (but failed). Lief navigatieteam, Harald, Wout, Nikie, Jasper en Susan, bedankt voor alle hulp en tijd die jullie in de twee navigatiestudies van dit boekje hebben gestoken. Jullie zijn stuk voor stuk fijne mensen! Want naast het feit dat de samenwerking altijd goed ging, gingen het borrelen nog beter. Ik hoop dat ik nog vaak mag aansluiten bij de borrels en 
dat het nog vaak mag escaleren. Lisanne and Behdad, the sports and the cake/sweets lover. I'm happy you both moved for the U-building to our hallway. Lunches became much more fun with you. Thank you for all the cookies and the nice conversations. And remember: 'stressed' is 'desserts' spelled backwards. Esther, wat ben ik blij dat ik jou beter heb leren kennen. We hebben in het laatste jaar echt een band opgebouwd. Ik vond samen onze PhD's afronden en tegelijkertijd zwanger zijn heel bijzonder. Ik kom jullie sowieso opzoeken in Amersfoort. Bart, jij kwam er pas aan het eind van mijn PhD bij maar was meteen een aanwinst voor onze donderdagochtenden. Ik hoop dat je een mooie tijd tegemoet gaat in Zurich of waar je ook terecht komt. Roeland, de rectum navigatiestudie was niet makkelijk maar ik ben blij dat we het tot zo'n goed einde hebben gebracht. Succes met de laatste loodjes en veel plezier met reizen. Jasper, dank voor je kritische blik en je altijd snelle reactie op mijn mailtjes en artikelen. Jouw chocoladebroodjes traditie hebben we met veel plezier voortgezet. En dan tot slot, lieve Sandra, dankjewel voor alle hulp tijdens mijn promotie. Jouw creativiteit met Theo's agenda heeft mijn tijd in het AvL een stuk gemakkelijker gemaakt.

Lieve jaargenootjes, lieve Marie-Sophie, Samantha, Ilse, Margreet, Hanna en Frederique, wat hebben wij veel meegemaakt sinds we elkaar kennen. Bedankt voor de fantastische jaren vol festivals, etentjes, borrels en mooie reisjes! Ik had het voor geen goud willen missen. Ik ben blij dat we elkaar nog zo vaak zien en hoop dat dat nog lang zo mag blijven. Ik kijk uit naar ons volgende tripje.

Lieve co-genootjes en dan speciaal lieve Mariska, Nina en Anna, met jullie kan ik heerlijk lang en ongegeneerd praten over het leven in het ziekenhuis en als PhDer. Bedankt voor jullie luisterend oor en alle gezellige koffietjes, etentjes en borrels. Jullie zijn lieverds!

Liza, hoeveel dagen hebben wij samen brak doorgebracht in onze studententijd? Ondanks het feit dat we heel ander werk doen, begrijp je mijn struggles en hoogtepunten en weet je altijd de goede vragen te stellen. Ik waardeer je ongezouten mening en hoop dat we nog lang vriendinnetjes blijven!

Lieve Anne, onze levens hebben de afgelopen 12 jaar zowat synchroon gelopen en het was een feestje om dit altijd met je te delen! We vierden samen dat we waren ingeloot, lid waren, tegelijkertijd afstudeerden, onze eerste baan kregen en 2 weken na elkaar waren uitgerekend. En nu dat we allebei een prachtige baby hebben gekregen en volgend jaar gaan trouwen! Ik hoop dat onze kinderen vriendjes worden. 
Lieve Ank en Peter, bedankt voor de altijd oprechte interesse in mijn onderzoek en de steun die ik vanuit jullie heb gevoeld.

Lieve papa en mama, bedankt voor jullie onvoorwaardelijke steun, liefde, hulp en trots. Ik weet dat ik met alles bij jullie terecht kan, of het nou over werk of prive gaat. Door jullie betrokkenheid voelt het altijd alsof ik de wind in de rug heb en daar kan ik jullie niet dankbaar genoeg voor zijn! Lieve Eric en Judith, wat ben ik blij met de hechte band die wij hebben. Er gaat geen week voorbij dat we elkaar niet spreken en ik hou van de chaos die die er ontstaat als we allemaal samen zijn! Jullie zijn toppers!

Lieve David, nog zo klein maar al zo belangrijk in ons leven! Ik kan de hele dag naar je kijken zonder verveeld te raken. Jouw lieve glimlach en kusjes maken de gebroken nachten goed.

Lieve Willem, er bestaan geen woorden die goed beschrijven wat jij voor mij betekent! Wat ben ik blij dat je bij onze eerste date toch de moed hebt gehad om het terras op te komen lopen. Je haalt het beste in me naar boven en weet me altijd aan het lachen te maken. Ik ben zo trots op wie we zijn geworden in de afgelopen jaren! Ik geniet van onze gekke liedjes en dansjes. Ik hoop dat we elkaar nog lang blijven inspireren, motiveren en uitdagen want iedere dag met jou is een feestje. 


\section{Curriculum Vitae}

Esther is op 19 oktober 1989 geboren in Amersfoort en opgegroeid in Soest. Na het VWO op het Baarnsch Lyceum te hebben afgerond in 2008, is ze in Amsterdam gaan studeren. Het eerste jaar in Amsterdam heeft ze Biomedische Wetenschappen aan de Vrije Universiteit gestudeerd en in 2009 is ze daar toegelaten tot de opleiding Geneeskunde. Tijdens haar master is de liefde en passie voor het vak chirurgie ontstaan. Als chirurgie coassistent in Zuid-Afrika heeft ze veel ervaring opgedaan in de ok en een fantastische tijd

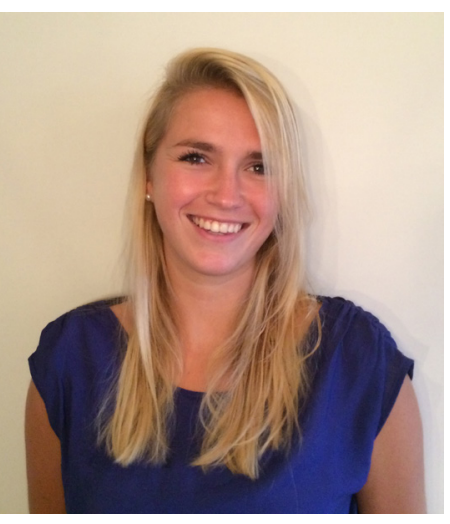
gehad. Het laatste jaar van haar master heeft ze daarom een 'schakelstage' gedaan: een traject waarbij ze het volledige jaar bij de chirurgie heeft doorgebracht. Aansluitend op haar wetenschappelijke stage in het Antoni van Leeuwenhoek is ze in augustus 2017 begonnen aan een PhD traject. Na haar PhD verdediging is ze van plan om, na een periode als ANIOS (arts niet in opleiding tot specialist) gewerkt te hebben, de opleiding chirurgie te gaan volgen.

Esther was born on October 19, 1989 in Amersfoort. She grew up in Soest where she went to primary school. After graduating in 2008 from Het Baarnsch Lyceum, she moved to Amsterdam. The first year she studied Biomedical Science at VU University and in 2009 she was accepted to study Medicine there. During her internships, she developed a love and passion for surgery. She had a fantastic time during her surgical internship in South Africa, which offered her the opportunity to gain a lot of experience in the OR. Therefore, she spent the final year of her masters in surgery, with a research internship at the department of Surgical Oncology in The Netherlands Cancer Institute - Antoni van Leeuwenhoek (NKI-AVL). She obtained her medical degree in July 2017 and started subsequently as a PhD candidate in the NKI-AVL. After her PhD defence, she plans to apply for the surgical training. 